\title{
Multiphase transport model for relativistic heavy ion collisions
}

\author{
Zi-Wei Lin* \\ Physics Department, The Ohio State University, Columbus, Ohio 43210, USA \\ Che Ming Ko \\ Cyclotron Institute and Physics Department, Texas A $6 M$ University, College Station, Texas 77843, USA \\ Bao-An Li and Bin Zhang \\ Department of Chemistry and Physics, Arkansas State University, State University, Arkansas 72467, USA \\ Subrata Pal \\ Department of Physics, Michigan State University, East Lansing, Michigan 48824, USA
}

(Received 30 November 2004; published 8 December 2005)

\begin{abstract}
We describe in detail how the different components of a multiphase transport (AMPT) model that uses the heavy ion jet interaction generator (HIJING) for generating the initial conditions, Zhang's parton cascade (ZPC) for modeling partonic scatterings, the Lund string fragmentation model or a quark coalescence model for hadronization, and a relativistic transport (ART) model for treating hadronic scatterings are improved and combined to give a coherent description of the dynamics of relativistic heavy ion collisions. We also explain the way parameters in the model are determined and discuss the sensitivity of predicted results to physical input in the model. Comparisons of these results to experimental data, mainly from heavy ion collisions at the BNL Relativistic Heavy Ion Collider, are then made in order to extract information on the properties of the hot dense matter formed in these collisions.
\end{abstract}

DOI: 10.1103/PhysRevC.72.064901

PACS number(s): 25.75.-q, 12.38.Mh, 24.10.Lx

\section{INTRODUCTION}

Colliding heavy ions at relativistic energies makes it possible to subject nuclear matter to the extreme condition of large compression, leading to energy densities that can exceed that for producing a plasma of deconfined quarks and gluons, which is believed to have existed during the first microsecond after the Big Bang. Experiments at the BNL Relativistic Heavy Ion Collider (RHIC) with center-of-mass energy up to $\sqrt{s_{N N}}=200 \mathrm{GeV}$ in $\mathrm{Au}+\mathrm{Au}$ collisions thus provide the opportunity to study the properties of this so-called quark-gluon plasma (QGP). At the future Large Hadron Collider (LHC) at CERN, which will allow $\mathrm{Pb}+\mathrm{Pb}$ collisions at $\sqrt{s_{N N}}=5.5 \mathrm{TeV}$, the produced quark-gluon plasma will have an even higher temperature and a nearly vanishing net baryon chemical potential.

Many observables have been measured at RHIC, such as the rapidity distributions of various particles and their transverse momentum spectra up to very high transverse momentum, the centrality dependence of these observables, and the elliptic flows of various particles, as well as both identical and nonidentical two-particle correlations. To understand these extensive experimental results, many theoretical models have been introduced. They range from thermal models [1-4] based on the assumption of global thermal and chemical equilibrium, to hydrodynamic models [5-11] based only on the assumption of local thermal equilibrium, to transport models [12-26] that treat nonequilibrium dynamics explicitly. The thermal

\footnotetext{
*Present address: 301 Sparkman Drive, VBRH E-39, University of Alabama in Huntsville, Huntsville, AL 35899.
}

models have been very successful in accounting for the yield of various particles and their ratios, while the hydrodynamic models are particularly useful for understanding the collective behavior of low transverse momentum particles such as the elliptic flow [8-11]. Since transport models treat chemical and thermal freeze-out dynamically, they are also natural and powerful tools for studying the Hanbury-Brown-Twiss interferometry of hadrons. For hard processes that involve large momentum transfer, approaches based on the perturbative quantum chromodynamics (pQCD) using parton distribution functions in the colliding nuclei have been used [27,28]. Also, the classical Yang-Mills theory has been developed to address the evolution of parton distribution functions in nuclei at ultrarelativistic energies [29-31] and used to study the hadron rapidity distribution and its centrality dependence at RHIC [32-34]. These problems have also been studied in the pQCD-based final-state saturation model [35-37].

Although studies based on the pQCD [38] have shown that thermalization could be achieved in collisions of very large nuclei and/or at extremely high energy, even though the strong coupling constant at the saturation scale is asymptotically small, the dense matter created in heavy ion collisions at RHIC may, however, not achieve full thermal or chemical equilibrium as a result of its finite volume and energy. To address such nonequilibrium many-body dynamics, we have developed a multiphase transport (AMPT) model that includes both initial partonic and final hadronic interactions and the transition between these two phases of matter [39-50]. The AMPT model is constructed to describe nuclear collisions ranging from $p+A$ to $A+A$ systems at center-of-mass energies from about $\sqrt{s_{N N}}=5$ to $5500 \mathrm{GeV}$ at LHC, where 
strings and minijets dominate the initial energy production and effects from final-state interactions are important. For the initial conditions, the AMPT model uses the hard minijet partons and soft strings from the heavy ion jet interaction generator (HIJING) model. Zhang's parton cascade (ZPC) is then used to describe scatterings among partons, which is followed by a hadronization process based on the Lund string fragmentation model or by a quark coalescence model. The latter is introduced for an extended AMPT model with string melting in which hadrons, which would have been produced from string fragmentation, are converted instead to their valence quarks and antiquarks. Scatterings among the resulting hadrons are described by a relativistic transport (ART) model. With parameters, such as those in the string fragmentation, fixed by the experimental data from heavy ion collisions at the CERN super proton synchrotron (SPS), the AMPT model has been able to reasonably describe many of the experimental observations at RHIC.

In this paper, we give a detailed description of the different components of the AMPT model, discuss the parameters in the model, show the sensitivity of its results to the input to the model, and compare its predictions with experimental data. The paper is organized as follows. In Sec. II, we describe the different components of the AMPT model: The HIJING model and string melting, the ZPC model, the Lund string fragmentation model, the quark coalescence model used for the scenario of string melting, and the extended ART model. Tests of the AMPT model against data from $p p$ and $p \bar{p}$ reactions are given in Sec. III. Results from the AMPT model for heavy ion collisions at SPS energies are discussed in Sec. IV for hadron rapidity distributions and transverse momentum spectra, baryon stopping, and antiproton production. In Sec. V, we show results at RHIC for hadron rapidity distributions and transverse momentum spectra, particle ratios, baryon and antibaryon production, and the production of multistrange baryons as well as $J / \psi$. We further show results from the AMPT model with string melting on hadron elliptic flows and two-pion interferometry at RHIC. In Sec. VI, we present the AMPT predictions for hadron rapidity and transverse momentum distributions in $\mathrm{Pb}+\mathrm{Pb}$ collisions at the $\mathrm{LHC}$ energy. Discussions on possible future improvements of the AMPT model are presented in Sec. VII, and a summary is finally given in Sec. VIII.

\section{THE AMPT MODEL}

The AMPT model consists of four main components: the initial conditions, partonic interactions, conversion from the partonic to the hadronic matter, and hadronic interactions. The initial conditions, which include the spatial and momentum distributions of minijet partons and soft string excitations, are obtained from the HIJING model [51-54]. Currently, the AMPT model uses the HIJING model version 1.383 [55], which does not include baryon junctions [56]. Scatterings among partons are modeled by ZPC [18], which at present includes only two-body scatterings with cross sections obtained from the pQCD with screening masses. In the default AMPT model [39-44,46,47,49], partons are recombined with their parent strings when they stop interacting, and the resulting strings

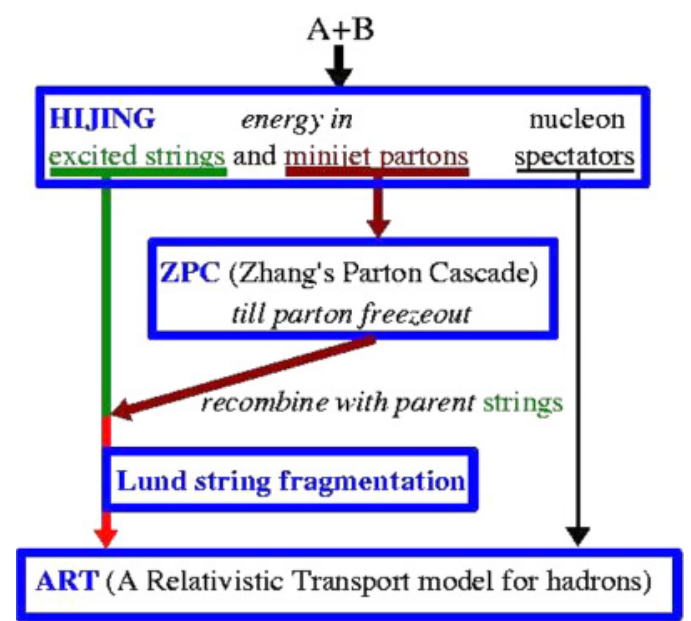

FIG. 1. (Color online) Structure of the default AMPT model.

are converted to hadrons using the Lund string fragmentation model [57-59]. In the AMPT model with string melting $[45,48,50]$, a quark coalescence model is used instead to combine partons into hadrons. The dynamics of the subsequent hadronic matter is described by a hadronic cascade, which is based on the ART model [14,25] and extended to include additional reaction channels that are important at high energies. These channels include the formation and decay of $K^{*}$ resonance and antibaryon resonances and baryon-antibaryon production from mesons and their inverse reactions of annihilation. Final results from the AMPT model are obtained after hadronic interactions are terminated at a cutoff time $t_{\text {cut }}$ when observables under study are considered to be stable, i.e., when further hadronic interactions after $t_{\text {cut }}$ will not significantly affect these observables. We note that two-body partonic scatterings at all possible times have been included because the algorithm of ZPC, which propagates partons directly to the time when the next collision occurs, is fundamentally different from the fixed time step method used in the ART model.

In Figs. 1 and 2, we show, respectively, the schematic structures of the default AMPT model [39-44,46,47] and the AMPT model with string melting $[45,48,50]$ described above. The full source code of the AMPT model in the FORTRAN 77 language and instructions for users are available online at the OSCAR [60] and EPAPS [61] websites. The default AMPT model is named version 1.x, and the AMPT model with string melting is named version 2.y; the value of the integer extension $x$ or $y$ increases whenever the source code is modified. Current versions of the AMPT models are 1.11 for the default model and 2.11 for the string melting model. In the following, we explain in detail each of the four components of the AMPT model and the way they are combined to describe relativistic heavy ion collisions.

\section{A. Initial conditions}

\section{The default AMPT model}

In the default AMPT model, initial conditions for heavy ion collisions at RHIC are obtained from the HIJING model [51-54]. In this model, the radial density profiles of the two 


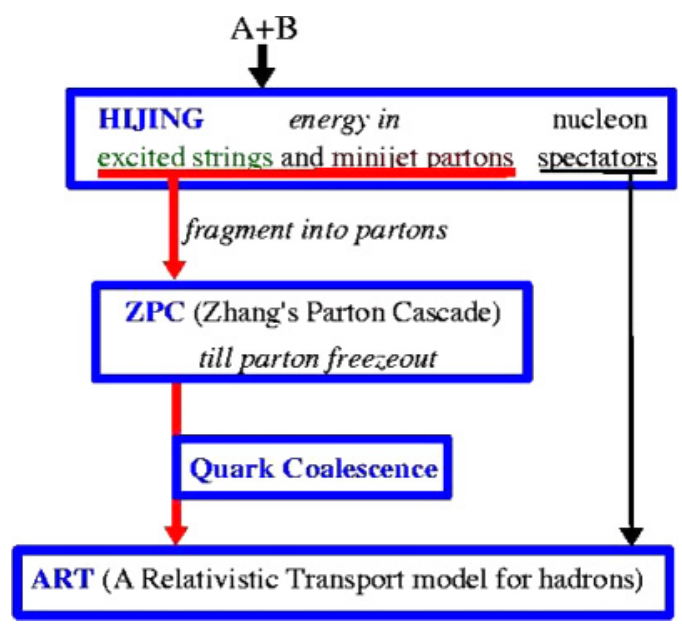

FIG. 2. (Color online) Structure of the AMPT model with string melting.

colliding nuclei are taken to have Woods-Saxon shapes, and multiple scatterings among incoming nucleons are treated in the eikonal formalism. Particle production from two colliding nucleons is described in terms of a hard and a soft component. The hard component involves processes in which the momentum transfer is larger than a cutoff momentum $p_{0}$ and is evaluated by the $\mathrm{pQCD}$ using the parton distribution function in a nucleus. These hard processes lead to the production of energetic minijet partons and are treated via the PYTHIA program. The soft component, on the other hand, takes into account non-perturbative processes with momentum transfer below $p_{0}$ and is modeled by the formation of strings. The excited strings are assumed to decay independently according to the Lund JETSET fragmentation model.

From the $p p$ and $p \bar{p}$ total cross sections and the ratio of $\sigma_{\mathrm{el}} / \sigma_{\text {tot }}$ in the energy range $20<\sqrt{s}<1800 \mathrm{GeV}$, it has been found that the experimental data can be fitted with a nucleon-nucleon soft cross section $\sigma_{s}(s)=57 \mathrm{mb}$ at high energies and $p_{0}=2 \mathrm{GeV} / c$ [51]. The independence of these two parameters on the colliding energy is due to the use of the Duke-Owens set 1 for the parton distribution function [62] in the nucleon. With different parton distribution functions, an energy-dependent $p_{0}$ may be needed to fit the same $p p$ and $p \bar{p}$ data $[63,64]$. We note that since the number of hard collisions in an $A+A$ collision roughly scales as $A^{4 / 3}$ and grows fast with colliding energy while the number of strings roughly scales as $A$, minijet production becomes more important as the energy of heavy ion collisions increases [51,65].

Because of nuclear shadowing, both quark [66] and gluon [67] distribution functions in nuclei are different from the simple superposition of their distributions in a nucleon. This effect has been included in the HIJING model via the following impact-parameter-dependent but $Q^{2}$ (and flavor)-independent parametrization [52]:

$$
\begin{aligned}
R_{A}(x, r) \equiv & \frac{f_{a}^{A}\left(x, Q^{2}, r\right)}{A f_{a}^{N}\left(x, Q^{2}\right)} \\
= & 1+1.19 \ln ^{1 / 6} A\left(x^{3}-1.2 x^{2}+0.21 x\right) \\
& -\left[\alpha_{A}(r)-\frac{1.08\left(A^{1 / 3}-1\right) \sqrt{x}}{\ln (A+1)}\right] e^{-x^{2} / 0.01},
\end{aligned}
$$

where $x$ is the light-cone momentum fraction of parton $a$, and $f_{a}$ is the parton distribution function. The impact-parameter dependence of the nuclear shadowing effect is controlled by

$$
\alpha_{A}(r)=0.133\left(A^{1 / 3}-1\right) \sqrt{1-r^{2} / R_{A}^{2}},
$$

with $r$ denoting the transverse distance of an interacting nucleon from the center of the nucleus with radius $R_{A}=$ $1.2 A^{1 / 3}$. Note that there is a modified HIJING model which uses a different parametrization for the nuclear shadowing that is also flavor dependent [63].

To take into account the Lorentz boost effect, we have introduced a formation time for minijet partons that depends on their four momenta [68]. Specifically, the formation time for each parton in the default AMPT model is taken to have a Lorentzian distribution with a half width $t_{f}=E / m_{T}^{2}$, where $E$ and $m_{T}$ are the parton energy and transverse mass, respectively. Initial positions of formed minijet partons are calculated from those of their parent nucleons using straight-line trajectories.

\section{The AMPT model with string melting}

Although the partonic part in the default AMPT model includes only minijets from the HIJING model, its energy density can be very high in heavy ion collisions at RHIC. As shown in Fig. 3 for the time evolutions of the energy and number densities of partons and hadrons in the central cell of central $(b=0 \mathrm{fm}) \mathrm{Au}+\mathrm{Au}$ collisions at $\sqrt{s_{N N}}=$ $200 \mathrm{GeV}$ in the center-of-mass frame, the partonic energy density during the first few $\mathrm{fm} / \mathrm{c}$ 's of the collision is more than an order of magnitude higher than the critical energy density $\left(\sim 1 \mathrm{GeV} / \mathrm{fm}^{3}\right)$ for the QCD phase transition, similar to that predicted by the high-density QCD approach [69]. The sharp increase in energy and number densities at about $3 \mathrm{fm} / c$ is due to the exclusion of energies that are associated with the excited strings in the partonic stage. Keeping strings in the high-energy-density region [70] thus underestimates the

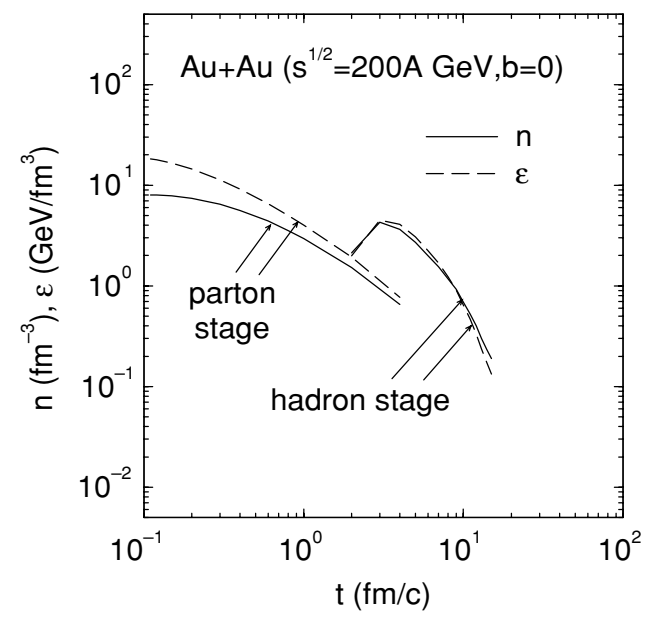

FIG. 3. Energy and number densities of minijet partons and formed hadrons in the central cell as functions of time for central $(b=0 \mathrm{fm}) \mathrm{Au}+\mathrm{Au}$ collisions at $\sqrt{s_{N N}}=200 \mathrm{GeV}$ from the default AMPT model, where the energy stored in the excited strings is absent in the parton stage and is released only when hadrons are formed. 
partonic effect in these collisions. We note that the central cell in the above calculation is chosen to have a transverse radius of $1 \mathrm{fm}$ and a longitudinal dimension between $-0.5 t$ and $0.5 t$, where time $t$ starts when the two nuclei are fully overlapped in the longitudinal direction.

To model the above effect in high-energy-density regions, we extend the AMPT model to include the string melting mechanism $[45,48,50]$, i.e, all excited strings that are not projectile and target nucleons without any interactions are converted to partons according to the flavor and spin structures of their valence quarks. In particular, a meson is converted to a quark and an antiquark, while a baryon is first converted to a quark and a diquark with weights according to relations from the $S U(6)$ quark model [71], and the diquark is then decomposed into two quarks. The quark and diquark masses are taken to be the same as in the PYTHIA program [59], e.g., $m_{u}=5.6, m_{d}=9.9$, and $m_{s}=199 \mathrm{MeV} / c^{2}$. We further assume that the above two-body decomposition is isotropic in the rest frame of the parent hadron or diquark, and the resulting partons do not undergo scatterings until after a formation time given by $t_{f}=E_{H} / m_{T, H}^{2}$, with $E_{H}$ and $m_{T, H}$ denoting the energy and transverse mass of the parent hadron. Similar to the case of minijet partons in the default AMPT model, initial positions of the partons from melted strings are calculated from those of their parent hadrons using straight-line trajectories.

The above formation time for partons is introduced to represent the time needed for their production from strong color fields. Although we consider hadrons before string melting as a convenient step in modeling the string melting process, choosing a formation time that depends on the momentum of the parent hadron ensures that partons from the melting of the same hadron would have the same formation time. The advantage of this choice is that the AMPT model with string melting reduces to HIJING results in the absence of partonic and hadronic interactions as these partons would then find each other as closest partners at the same freeze-out time and thus coalesce back to the original hadron. We note that the typical string fragmentation time of about 1 $\mathrm{fm} / c$ is not applied to the melting of strings because the fragmentation process involved here is considered as just an intermediate step in modeling parton production from the energy field of the strings in an environment of high energy density.

\section{B. Parton cascade}

In the transport approach, interactions among partons are described by equations of motion for their Wigner distribution functions, which describe semiclassically their density distributions in phase space. These equations can be approximately written as the following Boltzmann equations:

$$
\begin{aligned}
p^{\mu} \partial_{\mu} f_{a}(\mathbf{x}, \mathbf{p}, t)= & \sum_{m} \sum_{b_{1}, b_{2}, \cdots, b_{m}} \int \prod_{i=1}^{m} \frac{d^{3} p_{b_{i}}}{(2 \pi)^{3} 2 E_{b_{i}}} f_{b_{i}}\left(\mathbf{x}, \mathbf{p}_{\mathbf{b}_{\mathbf{i}}}, t\right) \\
& \times \sum_{n} \sum_{c_{1}, c_{2}, \cdots, c_{n}} \int \prod_{j=1}^{n} \frac{d^{3} p_{c_{j}}}{(2 \pi)^{3} 2 E_{c_{j}}}\left|M_{m \rightarrow n}\right|^{2}
\end{aligned}
$$

$$
\begin{aligned}
& \times(2 \pi)^{4} \delta^{4}\left(\sum_{k=1}^{m} p_{b_{k}}-\sum_{l=1}^{n} p_{c_{l}}\right) \\
& \times\left[-\sum_{q=1}^{m} \delta_{a b_{q}} \delta^{3}\left(\mathbf{p}-\mathbf{p}_{\mathbf{b}_{\mathbf{q}}}\right)\right. \\
& \left.+\sum_{r=1}^{n} \delta_{a c_{r}} \delta^{3}\left(\mathbf{p}-\mathbf{p}_{\mathbf{c}_{\mathbf{r}}}\right)\right]
\end{aligned}
$$

In the above, $f_{a}(\mathbf{x}, \mathbf{p}, t)$ is the distribution function of parton type $a$ at time $t$ in the phase space, and $M_{m \rightarrow n}$ denotes the matrix element of the multiparton interaction $m \rightarrow n$. If one considers only two-body interactions, these equations reduce to

$$
p^{\mu} \partial_{\mu} f(\mathbf{x}, \mathbf{p}, t) \propto \int \sigma f\left(\mathbf{x}_{1}, \mathbf{p}_{1}, t\right) f\left(\mathbf{x}_{\mathbf{2}}, \mathbf{p}_{2}, t\right),
$$

where $\sigma$ is the cross section for partonic two-body scattering, and the integral is evaluated over the momenta of the other three partons with the integrand containing factors such as a $\delta$ function for momentum conservation.

The Boltzmann equations are solved using ZPC [18], in which two partons undergo scattering whenever they approach each other with a closest distance smaller than $\sqrt{\sigma / \pi}$. At present, ZPC includes only parton two-body scattering such as $g g \rightarrow g g$ with cross sections calculated from the pQCD. For gluon elastic scattering, the leading-order QCD gives

$$
\begin{aligned}
\frac{d \sigma_{g g}}{d t} & =\frac{9 \pi \alpha_{s}^{2}}{2 s^{2}}\left(3-\frac{u t}{s^{2}}-\frac{u s}{t^{2}}-\frac{s t}{u^{2}}\right) \\
& \simeq \frac{9 \pi \alpha_{s}^{2}}{2}\left(\frac{1}{t^{2}}+\frac{1}{u^{2}}\right),
\end{aligned}
$$

where $\alpha_{s}$ is the strong coupling constant, and $s, t$ and $u$ are standard Mandelstam variables for elastic scattering of two partons. The second line in the above equation is obtained by keeping only the leading divergent terms. Since the scattering angle ranges from 0 to $\pi / 2$ for identical particles, one then has [18]

$$
\frac{d \sigma_{g g}}{d t} \simeq \frac{9 \pi \alpha_{s}^{2}}{2 t^{2}}
$$

if the scattering angle is between 0 and $\pi$.

The singularity in the total cross section can be regulated by a Debye screening mass $\mu$, leading to

$$
\begin{aligned}
\frac{d \sigma_{g g}}{d t} & \simeq \frac{9 \pi \alpha_{s}^{2}}{2\left(t-\mu^{2}\right)^{2}}, \\
\sigma_{g g} & =\frac{9 \pi \alpha_{s}^{2}}{2 \mu^{2}} \frac{1}{1+\mu^{2} / s} .
\end{aligned}
$$

The screening mass $\mu$ is generated by medium effects and is thus related to the parton phase-space density. For the partonic system expected to be formed in $\mathrm{Au}+\mathrm{Au}$ collisions at $\mathrm{RHIC}$, the value of $\mu$ is on the order of one inverse fermi [18]. For massless partons in a plasma at temperature $T$, their average colliding energy is $\sqrt{s} \sim \sqrt{18} T$, thus $\mu<\sqrt{s}$ for $\mu=$ $3 \mathrm{fm}^{-1}$ leads to the requirement $T>141 \mathrm{MeV}$. Since $s>\mu^{2}$ generally holds in hot QGP, the following simplified relation 
between the total parton elastic scattering cross section and the screening mass is used in the ZPC [72]

$$
\sigma_{g g} \approx \frac{9 \pi \alpha_{s}^{2}}{2 \mu^{2}} .
$$

A value of $3 \mathrm{fm}^{-1}$ for the screening mass $\mu$ thus leads to a total cross section of about $3 \mathrm{mb}$ for the elastic scattering between two gluons. By changing the value of the screening mass $\mu$, different cross sections can be obtained, and this will be used in studying the effect of parton cross sections in heavy ion collisions at RHIC. This cross section is used in AMPT not only in the default model, which includes only scatterings of minijet gluons, but also in the string melting model, which only includes scatterings of quarks/antiquarks of all flavors. We have therefore neglected in the latter case the difference between the Casimir factors for quarks and gluons.

We note that minijet partons produced from hard scatterings in the HIJING model can lose energy by gluon splitting and transfer their energies to nearby soft strings. In the AMPT model, this so-called jet quenching in the HIJING model is replaced by parton scatterings in ZPC. Since only two-body scatterings are included in ZPC, higher-order contributions to the jet energy loss are still missing in the AMPT model.

\section{Hadronization}

Two different hadronization mechanisms are used in the AMPT model for the two different initial conditions introduced in Sec. II A. In the default AMPT model, minijets coexist with the remaining part of their parent nucleons, and together they form new excited strings after partonic interactions. Hadronization of these strings are described by the Lund string model. In the AMPT model with string melting, these strings are converted to soft partons, and their hadronization is based on a simple quark coalescence model, similar to that in the ALCOR model [73].

\section{Lund string fragmentation for the default AMPT model}

Hadron production from the minijet partons and soft strings in the default AMPT model is modeled as follows. After minijet partons stop interacting, i.e., after they no longer scatter with other partons, they are combined with their parent strings to form excited strings, which are then converted to hadrons according to the Lund string fragmentation model $[57,58]$. In the Lund model as implemented in the JETSET/PYTHIA routine [59], one assumes that a string fragments into quark-antiquark pairs with a Gaussian distribution in transverse momentum. A suppression factor of 0.30 is further introduced for the production of strange quark-antiquark pairs relative to that of light quark-antiquark pairs. Hadrons are formed from these quarks and antiquarks by using a symmetric fragmentation function [57,58]. Specifically, the transverse momentum of a hadron is given by those of its constituent quarks, while its longitudinal momentum is determined by the Lund symmetric fragmentation function [74]

$$
f(z) \propto z^{-1}(1-z)^{a} \exp \left(-b m_{\perp}^{2} / z\right),
$$

with $z$ denoting the light-cone momentum fraction of the produced hadron with respect to that of the fragmenting string. The average squared transverse momentum is then given by

$$
\begin{aligned}
\left\langle p_{\perp}^{2}\right\rangle & =\frac{\int p_{\perp}^{2} f(z) d^{2} p_{\perp} d z}{\int f(z) d^{2} p_{\perp} d z} \\
& =\frac{\int_{0}^{z_{\max }} z(1-z)^{a} \exp \left(-b m^{2} / z\right) d z}{b \int_{0}^{z_{\max }}(1-z)^{a} \exp \left(-b m^{2} / z\right) d z} .
\end{aligned}
$$

For massless particles, it reduces to

$$
\left\langle p_{\perp}^{2}\right\rangle=\frac{1}{b} \frac{\int_{0}^{1} z(1-z)^{a} d z}{\int_{0}^{1}(1-z)^{a} d z}=\frac{1}{b(2+a)} .
$$

Since quark-antiquark pair production from string fragmentation in the Lund model is based on the Schwinger mechanism [75] for particle production in strong field, its production probability is proportional to $\exp \left(-\pi m_{\perp}^{2} / \kappa\right)$, where $\kappa$ is the string tension, i.e., the energy in a unit length of string. Due to its large mass, strange quark production is suppressed by the factor $\exp \left[-\pi\left(m_{s}^{2}-m_{u}^{2}\right) / \kappa\right]$, compared to that of light quarks. Also, the average squared transverse momentum of produced particles is proportional to the string tension, i.e. $\left\langle p_{\perp}^{2}\right\rangle \propto \kappa$. Comparing this with Eq. (11), one finds that the two parameters $a$ and $b$ in the Lund fragmentation function are approximately related to the string tension by

$$
\kappa \propto \frac{1}{b(2+a)} .
$$

After production from string fragmentation, hadrons are given an additional proper formation time of $0.7 \mathrm{fm} / c$ [76]. Positions of formed hadrons are then calculated from those of their parent strings by following straight-line trajectories.

\section{Quark coalescence for the AMPT model with string melting}

After partons in the string melting scenario stop interacting, we model their hadronization via a simple quark coalescence model by combining the two nearest partons into a meson and the three nearest quarks (antiquarks) into a baryon (antibaryon). Since the invariant mass of combined partons forms a continuous spectrum instead of a discrete one, it is generally impossible to conserve four-momentum when partons are coalesced into a hadron. At present, we choose to conserve the three-momentum during coalescence and determine the hadron species according to the flavor and invariant mass of coalescing partons [77]. For pseudoscalar and vector mesons with same flavor composition, the meson with mass closer to the invariant mass of coalescing quark and antiquark pair is formed. For example, whether a $\pi^{-}$or a $\rho^{-}$is formed from the coalescence of a pair of $\bar{u}$ and $d$ quarks depends on whether the invariance mass of the quarks is closer to the $\pi^{-}$mass or the centroid of $\rho$ mass. The same criterion applies to the formation of octet and decuplet baryons that have same flavor composition. It is more complicated to treat the formation probabilities of flavor-diagonal mesons such as $\pi^{0}$ and $\eta$ in the pseudoscalar meson octet, $\rho^{0}$ and $\omega$ in the vector meson octet. Neglecting the mixing of $\eta$ meson with the $s \bar{s}$ state, we take the following approach for these flavor-diagonal mesons within the $\mathrm{SU}(2)$ flavor space. 
For $\pi^{0}$ formation from a $u \bar{u}$ or $d \bar{d}$ pair, the probability $P_{\pi^{0}}$ is determined from the average of the numbers of formed $\pi^{+}$and $\pi^{-}$mesons divided by the total number of $u \bar{u}$ and $d \bar{d}$ pairs. Thus the total number of $\pi^{0}, n_{\pi^{0}}$, is determined by applying the probability $P_{\pi^{0}}$ to each $u \bar{u}$ or $d \bar{d}$ pair. The probability $P_{\rho^{0}}$ for forming a $\rho^{0}$ meson from a $u \bar{u}$ or $d \bar{d}$ pair is determined by a similar procedure. After sorting all $u \bar{u}$ or $d \bar{d}$ pairs according to their invariant masses, the lightest $n_{\pi^{0}}$ pairs are assigned as $\pi^{0}$ mesons. The rest of $u \bar{u}$ or $d \bar{d}$ pairs form $\rho^{0}$ mesons according to the probability of $P_{\rho^{0}} /\left(1-P_{\pi^{0}}\right)$, and the remaining pairs form $\omega$ and $\eta$ mesons with equal probabilities.

The above quark coalescence model includes the formation of all mesons and baryons listed in the HIJING program [54] except $\eta^{\prime}, \Sigma^{*}$, and $\Xi^{*}$, which are not present in our hadronic transport model as well as $K_{S}^{0}$ and $K_{L}^{0}$ states. The resulting hadrons are given an additional formation time of $0.7 \mathrm{fm} / c$ in their rest frame before they are allowed to scatter with other hadrons during the hadron cascade. As partons freeze-out dynamically at different times in the parton cascade, hadron formation from their coalescence thus occurs at different times, leading to the appearance of a coexisting phase of partons and hadrons during hadronization.

\section{Hadron cascade}

In the AMPT model, the following hadrons with all possible charges are explicitly included: $\pi, \rho, \omega, \eta, K, K^{*}$, and $\phi$ for mesons; $N, \Delta, N^{*}(1440), N^{*}(1535), \Lambda, \Sigma, \Xi$, and $\Omega$ for baryons and corresponding antibaryons. Many other higher resonances are taken into account implicitly as intermediate states in scatterings between the above particles [14,25]. Interactions among these hadrons and corresponding inverse reactions are included as discussed in the following subsections.

\section{The ART model}

Hadron cascade in the AMPT model is based on the ART model [14,78], which is a relativistic transport model originally developed for heavy ion collisions at the alternating gradient synchrotron (AGS) energies. The ART model includes baryon-baryon, baryon-meson, and meson-meson elastic and inelastic scatterings. It treats explicitly the isospin degrees of freedom for most particle species and their interactions, making it suitable for studying isospin effects in heavy ion collisions [79]. Since it includes mean-field potentials for nucleons and kaons, the ART model can also be used for studying the effect due to the hadronic equation of state. Resonances such as $\rho$ and $\Delta$ are formed from pion-pion and pion-nucleon scattering, respectively, with cross sections given by the standard Breit-Wigner form, and they also decay according to their respective widths. In all calculations presented in this study, the masses and widths of resonances are taken to be their values in the vacuum; i.e., effects due to possible modifications in dense hadronic matter [80] are neglected. Also, we turned off the potentials in the AMPT model because their effects are much less important than scatterings in high-energy heavy ion collisions such as at SPS and RHIC.

For baryon-baryon scatterings, the ART model includes the following inelastic channels: $N N \leftrightarrow N\left(\Delta N^{*}\right), N N \leftrightarrow$
$\Delta\left(\Delta N^{*}(1440)\right), N N \leftrightarrow N N(\pi \rho \omega),(N \Delta) \Delta \leftrightarrow N N^{*}, \quad$ and $\Delta N^{*}(1440) \leftrightarrow N N^{*}(1535)$. In these, $N^{*}$ denotes either $N^{*}(1440)$ or $N^{*}(1535)$, and the symbol $\left(\Delta N^{*}\right)$ denotes a $\Delta$ or an $N^{*}$. Also included are reaction channels relevant to kaon production, i.e., $\left(N \Delta N^{*}\right)\left(N \Delta N^{*}\right) \rightarrow(N \Delta)(\Lambda \Sigma) K$. Details on their cross sections and the momentum dependence of resonance widths can be found in the original ART model [14].

For meson-baryon scatterings, the ART model includes the following reaction channels for the formation and decay of resonances: $\pi N \leftrightarrow\left(\Delta N^{*}(1440) N^{*}(1535)\right)$, and $\eta N \leftrightarrow N^{*}(1535)$. There are also elastic scatterings such as $(\pi \rho)\left(N \Delta N^{*}\right) \rightarrow(\pi \rho)\left(N \Delta N^{*}\right)$. For example, the cross section for the elastic scattering of $\pi^{0} N$ is evaluated by including heavier baryon resonances with masses up to $2.0 \mathrm{GeV} / c^{2}$ as intermediate states using the Breit-Wigner form but neglecting interferences between the amplitudes from different resonances [14]. The ART model further includes inelastic reaction channels such as $\pi N \leftrightarrow(\pi \rho \eta) \Delta$ and kaon production channels such as $(\pi \rho \omega \eta)\left(N \Delta N^{*}\right) \leftrightarrow$ $K(\Lambda \Sigma)$. Kaon elastic scatterings with nucleons and baryon resonances are included with a constant cross section of $10 \mathrm{mb}$ [14]. Antikaon elastic scatterings with nucleons and inelastic channels, such as $\bar{K}\left(N \Delta N^{*}\right) \leftrightarrow \pi(\Lambda \Sigma)$, are included [81] using parametrized experimental data [82]. Also included are kaon production channels involving three-body final states, $(\pi \rho \omega)\left(N \Delta N^{*}\right) \rightarrow K \bar{K} N$ [81]. Because of the difficulty associated with the three-body kinematics, the inverse kaon annihilation reactions of the above channels are neglected.

For meson-meson interactions, the ART model includes both elastic and inelastic $\pi \pi$ interactions, with the elastic cross section consisting of $\rho$ meson formation and the remaining part treated as elastic scattering. Kaon production from inelastic scatterings of light mesons is included via the reactions $(\pi \eta)(\pi \eta) \leftrightarrow K \bar{K}$ and $(\rho \omega)(\rho \omega) \leftrightarrow K \bar{K}$. Kaon or antikaon elastic scatterings with mesons in the SU(2) multiplets except the pion are included using a constant cross section of $10 \mathrm{mb}$ [14], while the kaon-pion elastic scattering is modeled through the $K^{*}$ resonance [42].

\section{Explicit inclusion of $K^{*}$ mesons}

The original ART model [14] includes the $K^{*}$ resonance implicitly through elastic $\pi K$ scattering with the standard Breit-Wigner form for the cross section [83], i.e., $\sigma_{\pi K}=$ $60 \mathrm{mb} /\left[1+4\left(\sqrt{s}-m_{K^{*}}\right)^{2} / \Gamma_{K^{*}}^{2}\right]$. Since the $K^{*}$ meson not only enhances elastic scattering between pion and kaon but also adds to strange particle production through its addition to the strangeness degeneracy, which becomes important when the hadronic matter is highly excited, $K^{*}$ and $\bar{K}^{*}$ are included explicitly in the hadronic phase of the AMPT model [42]. In addition to its formation from $\pi K$ scattering and its decay, elastic scatterings of $K^{*}$ with $(\rho \omega \eta)$ are included using the same constant cross section of $10 \mathrm{mb}$ as those used for kaons. Inelastic reaction channels of $(\pi \eta)(\rho \omega) \leftrightarrow K^{*} \bar{K}$ or $\bar{K}^{*} K$ and $\pi K \leftrightarrow K^{*}(\rho \omega)$ are also included [84].

\section{Baryon-antibaryon annihilation and production}

In heavy ion collisions at or above SPS energies, antibaryon production becomes significant and needs to be treated 
explicitly during hadron cascade. The AMPT model initially only included $N \bar{N}$ annihilation [40]. It was later extended to include $\left(N \Delta N^{*}\right)\left(\bar{N} \bar{\Delta} \bar{N}^{*}\right)$ annihilation and also the inverse reactions of baryon-antibaryon pair production from mesons [42].

The total cross section for $p \bar{p}$ annihilation is known empirically, and the data has been parametrized as [85]

$$
\sigma_{p \bar{p}}=67 \mathrm{mb} / p_{\text {lab }}^{0.7},
$$

where $p_{\text {lab }}$ in $\mathrm{GeV} / c$ is the proton momentum in the rest frame of the antiproton. Following Ref. [14], a maximum cross section of $400 \mathrm{mb}$ is imposed at low $p_{\text {lab. Using phase-space }}$ considerations [86], the branching ratios of $p \bar{p}$ annihilation to different multipion states are determined according to [86]

$$
M_{n}(\sqrt{s})=C\left[\frac{1}{6 \pi^{2}}\left(\frac{\sqrt{s}}{m_{\pi}}\right)^{3}\right]^{n} \frac{(4 n-4) !(2 n-1)}{(2 n-1) !^{2}(3 n-4) !},
$$

where $\sqrt{s}$ is the center-of-mass energy of the proton and antiproton, and $n$ is the number of pions in the final state. The dominant final states at moderate energies then involve several pions. For example, the branching ratios at $p_{\text {lab }}=$ $4 \mathrm{GeV} / c$ are $0.033,0.161,0.306,0.286,0.151,0.049$, and 0.011 , respectively, for $n$ from 3 to 9 . For baryon-antibaryon annihilation channels involving baryon resonances $\Delta$ or $N^{*}$, their annihilation cross sections and branching ratios are taken to be the same as for $p \bar{p}$ annihilation at the same center-of-mass energy.

To include the inverse reactions that produce baryonantibaryon pairs during hadron cascade, which currently only treats scattering of two particles, we have further assumed that the final state of three pions is equivalent to a $\pi \rho$ state; the four-pion final state is equivalent to $\rho \rho$ and $\pi \omega$ with equal probabilities; the five-pion state is equivalent to $\rho \omega$; and the six-pion state is equivalent to $\omega \omega$. The cross sections for baryon-antibaryon pair production from two mesons are then obtained from detailed balance relations. As shown later in the paper (Fig. 18), the above approximate treatment of antibaryon annihilation and production via two-meson states gives a satisfactory description of measured antiproton yield in central $\mathrm{Pb}+\mathrm{Pb}$ collisions at SPS.

\section{Multistrange baryon production from strangeness-exchange reactions}

Productions of multistrange baryons such as $\Xi$ and $\Omega$ are included in the AMPT model [44] through the strangenessexchange reactions such as

$$
\bar{K}(\Lambda \Sigma) \leftrightarrow \pi \Xi, \quad \bar{K} \Xi \leftrightarrow \pi \Omega .
$$

Since there is no experimental information on their cross sections, we have assumed that the matrix elements for $\bar{K}(\Lambda \Sigma) \rightarrow \pi \Xi$ and $\bar{K} \Xi \rightarrow \pi \Omega$ are the same as that for the reaction $\bar{K} N \rightarrow \pi \Sigma$ [87] at the same amount of energy above corresponding thresholds. The isospin-averaged cross section for the reaction $\bar{K} N \rightarrow \pi \Sigma$ can be related to the cross sections for the reactions $K^{-} p \rightarrow \Sigma^{0} \pi^{0}$ and $K^{-} n \rightarrow \Sigma^{0} \pi^{-}$, which are known empirically and have been parametrized in

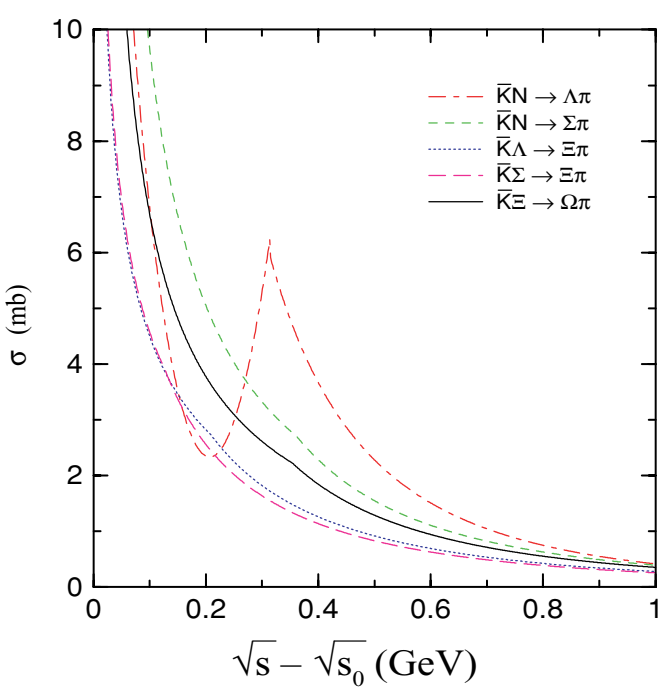

FIG. 4. (Color online) Isospin-averaged cross sections for multistrange baryon production as functions of c.m. energy of interacting antikaon and nucleon/hyperon.

Ref. [82], by

$$
\sigma_{\bar{K} N \rightarrow \Sigma \pi}=\frac{3}{2}\left(\sigma_{K^{-} p \rightarrow \Sigma^{0} \pi^{0}}+\sigma_{K^{-} n \rightarrow \Sigma^{0} \pi^{-}}\right) .
$$

In Fig. 4, this cross section as well as the isospin-averaged cross sections for other multistrange baryon production reactions are shown as functions of the center-of-mass energy above the threshold values of the interacting antikaon and nucleon/hyperon. The cross sections for the inverse multistrange baryon destruction reactions are then determined by detailed balance relations. We note that these cross sections are comparable to those predicted by the coupled-channel calculations based on the $\mathrm{SU}(3)$ invariant hadronic Lagrangian with empirical masses and coupling constants [88]. With these cross sections, the ART model is able to describe the measured $\Xi$ production in heavy ion collisions at the AGS energies [89]. We note that for strange baryons $\Lambda, \Xi, \Omega$ and their antiparticles, only their interactions with mesons have been included, while their annihilations by baryons have not been included in the AMPT model at present.

\section{5. $\phi$ meson production and scattering}

The AMPT model also includes $\phi$ meson formation from and decay to the kaon-antikaon pair with the formation cross section given by the standard Breit-Wigner form [47]. Inelastic scatterings of the $\phi$ meson include baryonbaryon channels, $\left(N \Delta N^{*}\right)\left(N \Delta N^{*}\right) \rightarrow \phi N N$, and mesonbaryon channels, $(\pi \rho)\left(N \Delta N^{*}\right) \leftrightarrow \phi\left(N \Delta N^{*}\right)$, where the cross sections for the forward-going reactions are taken from the one-boson-exchange model [90]. The meson-baryon channels also include $K(\Lambda \Sigma) \leftrightarrow \phi N$ with the cross section taken from a kaon-exchange model [91].

$\phi$ meson scatterings with mesons such as $\pi, \rho, K$, and $\phi$ have been studied before, and the total collisional width was found to be less than $35 \mathrm{MeV} / c^{2}$ [92]. A recent calculation based on the hidden local symmetry Lagrangian [93] shows, however, that the collisional rates of $\phi$ with pseudoscalar 
$(\pi, K)$ and vector $\left(\rho, \omega, K^{*}, \phi\right)$ mesons are appreciably larger. Assuming that the matrix elements are independent of centerof-mass energy, we have included all these possible reactions, i.e., $\phi(\pi \rho \omega) \leftrightarrow\left(K K^{*}\right)\left(\bar{K} \bar{K}^{*}\right)$ and $\phi\left(K K^{*}\right) \leftrightarrow(\pi \rho \omega)\left(K K^{*}\right)$, with cross sections determined from the partial collisional widths given in Ref. [93]. The cross section for the elastic scattering of the $\phi$ meson with a nucleon is set to $8 \mathrm{mb}$, while the $\phi$ meson elastic cross section with a meson is set to $5 \mathrm{mb}$. The value of $8 \mathrm{mb}$ is the $\phi N$ total cross section [91] estimated from the $\phi$ meson photoproduction data [94] and thus represents the upper bound on the $\phi$ meson elastic cross section with a nucleon; quark counting then gives $5 \mathrm{mb}$ as the upper bound on the $\phi$ meson elastic cross section with a meson. Note that in most calculations of our previous study on $\phi$ meson productions at SPS and RHIC [47], the elastic cross section for $\phi$ meson scattering with a nucleon was taken to be $0.56 \mathrm{mb}$ as extracted in Ref. [95] using the vector meson dominance model and the older $\phi$ meson photoproduction data; while based on results of Ref. [93], the $\phi K$ elastic cross section was extracted to be about $2 \mathrm{mb}$ [47], which was then used as the $\phi$ meson elastic cross section with other mesons [96].

\section{Other extensions}

Other extensions of the ART model have also been made in the hadronic phase of the AMPT model. Antibaryon resonances such as $\bar{\Delta}$ and $\bar{N}^{*}$ have been included explicitly with their formations, decays, and scatterings analogous to those of baryon resonances [44,48]. Also, inelastic meson-meson collisions such as $\pi \pi \leftrightarrow \rho \rho$ have been added, and elastic scatterings between $\pi$ and ( $\rho \omega \eta$ ) have been included with cross sections taken to be $20 \mathrm{mb}$. To address chemical equilibration of $\eta$ mesons, which affects the height (or the $\lambda$ parameter) of the correlation functions in two-pion interferometry, inelastic scatterings of $\eta$ meson with other mesons have also been included with a constant cross section of $5 \mathrm{mb}$ [48], which is roughly in line with recent theoretical predictions based on the hidden local symmetry Lagrangian [97].

\section{RESULTS FOR $p p$ AND $p \bar{p}$ COLLISIONS}

The default AMPT results with no popcorn mechanism (see discussions on the popcorn mechanism in Sec. IV B) for $p p$ and $p \bar{p}$ collisions are essentially the same as the results from the HIJING model. In this section, we compare the results from the default AMPT model with or without the popcorn mechanism against available data from $p p$ and $p \bar{p}$ collisions [98], where HIJING values for the $a$ and $b$ parameters, $a=0.5$ and $b=$ $0.9 \mathrm{GeV}^{-2}$, are used in Eq. (9) for the Lund fragmentation function.

\section{A. Rapidity distributions}

In Fig. 5, the AMPT results on charged-particle pseudorapidity distribution are compared with the UA5 data for $p \bar{p}$ collisions at $\sqrt{s}=200 \mathrm{GeV}$ [99]. Since the AMPT results given by the solid or the long-dashed curves represent all inelastic events with no trigger conditions, they should be compared with the UA5 inelastic data. The AMPT nonsingle-diffractive (NSD) results have included the UA5 NSD

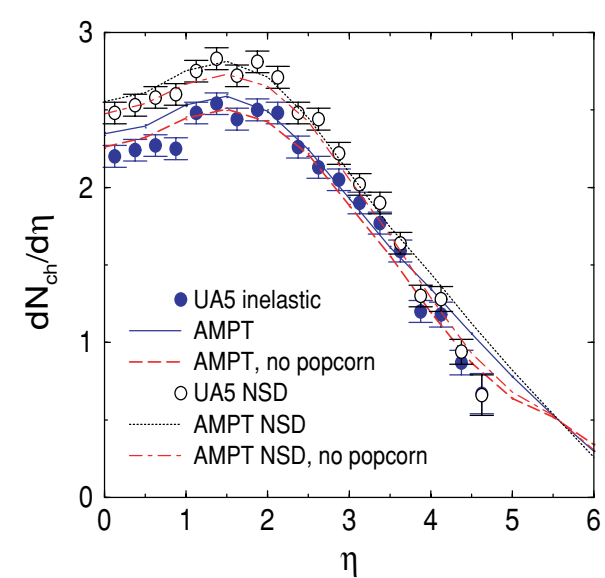

FIG. 5. (Color online) Pseudorapidity distributions of charged particles for $p \bar{p}$ collisions at $\sqrt{s}=200 \mathrm{GeV}$.

trigger by requiring events to have at least one charged particle each in both ends of the pseudorapidity intervals $2<|\eta|<5.6$ [99]. We see that the AMPT model with or without the popcorn mechanism agrees reasonably well with both NSD and inelastic data. The kaon rapidity distribution from the AMPT model is compared with the UA5 NSD data in Fig. 6, and both results with or without the popcorn mechanism also agree reasonably well with the data.

For $p p$ collisions at $P_{\text {lab }}=400 \mathrm{GeV} / c$, the rapidity distributions of pions, kaons, protons, and antiprotons are shown, respectively, in Figs. 7, 8, 9, and 10. Curves with circles are measured cross sections from the LEBC-EHS Collaboration [100], while for comparison the AMPT results have been scaled up by the inelastic cross section at this energy $\left(\sigma_{\text {inel }}=32 \mathrm{mb}\right)$. We see that similar descriptions of the charged-particle data are obtained with and without the popcorn mechanism. Including the popcorn mechanism, however, gives, a better description of measured pion, kaon, and antiproton yields and the shape of the proton rapidity distribution. We have thus included the popcorn mechanism in all AMPT calculations.

\section{B. Transverse momentum spectra}

The transverse momentum spectra of charged pions and protons for $p p$ collisions at $P_{\text {lab }}=24 \mathrm{GeV} / c$ from the AMPT

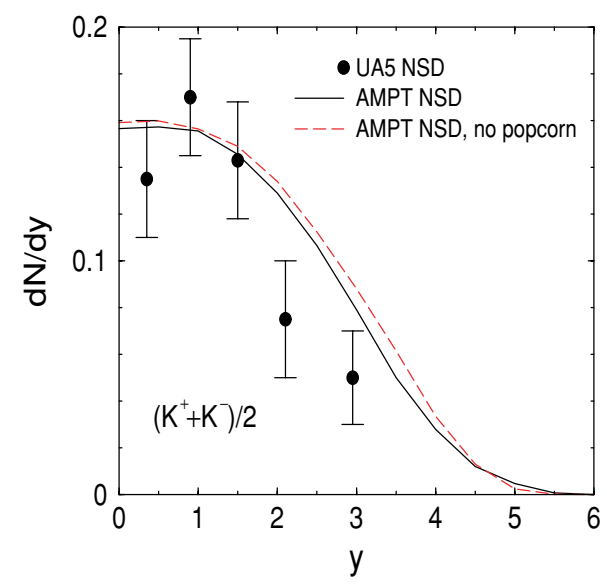

FIG. 6. (Color online) Same as Fig. 5 for kaons. 


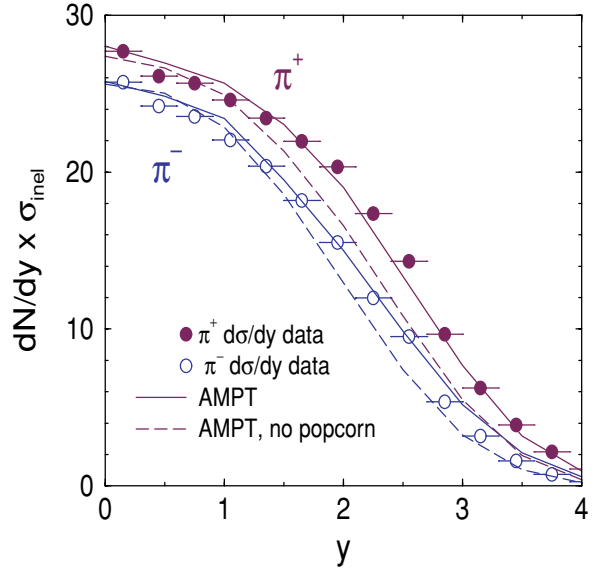

FIG. 7. (Color online) Rapidity distributions of pions for $p p$ collisions at $P_{\text {lab }}=400 \mathrm{GeV} / c$. Circles are data from the LEBCEHS Collaboration [100].

model are shown in Fig. 11. They are seen to reproduce reasonably well the experimental data from Ref. [101].

At the Tevatron energy of $\sqrt{s}=1.8 \mathrm{TeV}$, results from the AMPT model for the transverse momentum spectra of pions, kaons, and antiprotons are shown in Fig. 12 and compared with data from the E735 Collaboration [102]. The AMPT NSD results included the trigger by requiring events to have at least one charged particle each in both ends of the pseudorapidity intervals $3<|\eta|<4.5$ [103]. We see that measured momentum spectra except for antiprotons are reproduced reasonably well by the AMPT model. Note that the E735 $p_{\mathrm{T}}$ spectrum data shown in Fig. 12 have been averaged over rapidity $y$ from weighing each track by the rapidity range of the spectrometer [102], thus they are for $d^{2} N /\left(2 \pi p_{\mathrm{T}} d p_{\mathrm{T}} d y\right)$ instead for $d^{2} N /\left(2 \pi p_{\mathrm{T}} d p_{\mathrm{T}} d \eta\right)$ even though the E735 spectrometer covers the acceptance of $-0.36<\eta<1.0$.

\section{Energy dependence}

Figure 13 shows the energy dependence of $d N_{\mathrm{ch}} / d \eta$ at $\eta=0$ for $p p$ and $p \bar{p}$ collisions together with UA5 and CDF data at the Tevatron $[99,104]$. The AMPT NSD results above

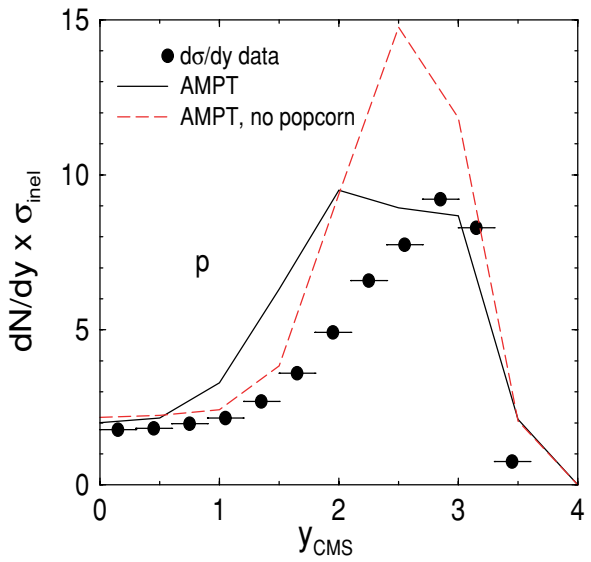

FIG. 9. (Color online) Same as Fig. 7 for protons.

$\sqrt{s}=1 \mathrm{TeV}$ included the CDF NSD trigger by requiring events to each have at least one charged particle in both ends of the pseudorapidity intervals of $3.2<|\eta|<5.9$ [104], and the AMPT NSD results below $\sqrt{s}=1 \mathrm{TeV}$ included the UA5 NSD trigger by requiring events to each have at least one charged particle in both ends of the pseudorapidity intervals of $2<|\eta|<5.6$ [99]. The agreement with the Tevatron data is reasonable.

The energy dependence of the full phase-space $K^{+} / \pi^{+}$ ratio for $p p$ collisions is shown in Fig. 14 together with the data compiled in Fig. 7 of Ref. [105]. It is seen that the AMPT model reproduces the data reasonably well.

The energy dependence of the mean transverse momenta of pions, kaons, and antiprotons are shown in Fig. 15, where filled circles represent the NSD data from the E735 Collaboration [106] and open circles represent data from the CERN intersecting storage rings (ISR) [107]. Note that the AMPT NSD results shown in Fig. 15 included the E735 NSD trigger [103] by requiring events to each have at least one charged particle in both ends of the pseudorapidity intervals $3<|\eta|<4.5$. However, the trigger condition for the CERN ISR data is different. From the comparison with the AMPT inelastic results (solid curves), we see that NSD triggers have larger effects at lower energies.

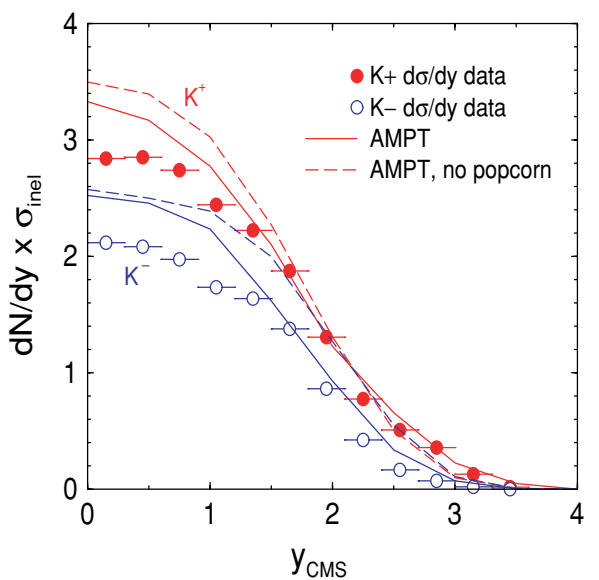

FIG. 8. (Color online) Same as Fig. 7 for kaons.

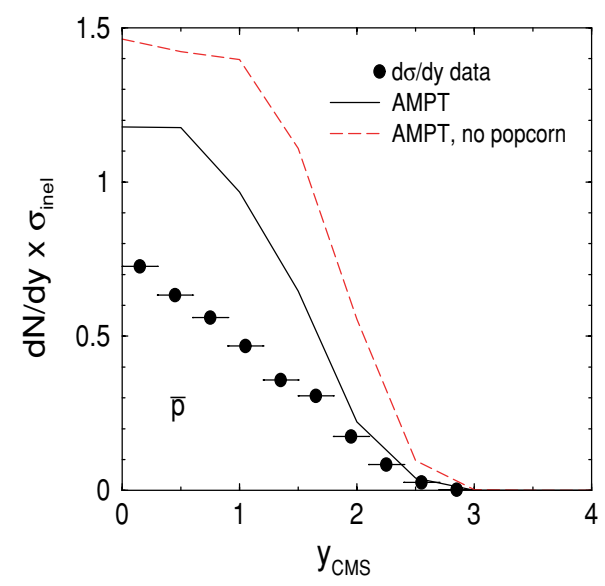

FIG. 10. (Color online) Same as Fig. 7 for antiprotons. 


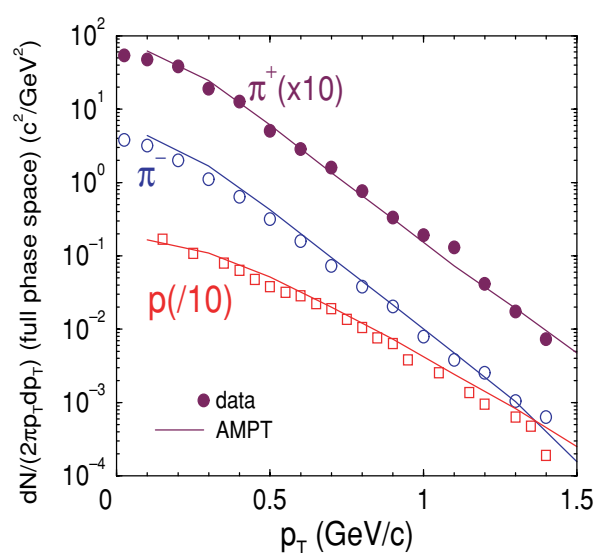

FIG. 11. (Color online) Transverse momentum spectra of charged pions and protons for $p p$ collisions at $P_{\mathrm{lab}}=24 \mathrm{GeV} / c$ with data from Ref. [101].

\section{RESULTS AT SPS ENERGIES}

To make predictions for heavy ion collisions at RHIC, we first use the AMPT model to study heavy ion collisions at SPS. In particular, parameters in the AMPT model are determined by fitting the experimental data from central $\mathrm{Pb}+\mathrm{Pb}$ collisions at the laboratory energy of $158 \mathrm{~A} \mathrm{GeV}$, corresponding to a center-of-mass energy of about $\sqrt{s_{N N}}=17.3 \mathrm{GeV}$.

\section{A. Rapidity distributions}

In Fig. 16, we show the rapidity distributions of negatively charged particles (upper left panel), net-protons and antiprotons (upper right panel), charged pions (lower left panel), and charged kaons (lower right panel) in central $(b \leqslant 3 \mathrm{fm}) \mathrm{Pb}+\mathrm{Pb}$ collisions at SPS, obtained from the AMPT model with the default values of $a=0.5$ and $b=0.9 \mathrm{GeV}^{-2}$ in the HIJING model for the string fragmentation function. Compared with experimental data for the $5 \%$ most central $\mathrm{Pb}+\mathrm{Pb}$ collisions from the NA49 Collaboration [105,108,109], it is seen that the

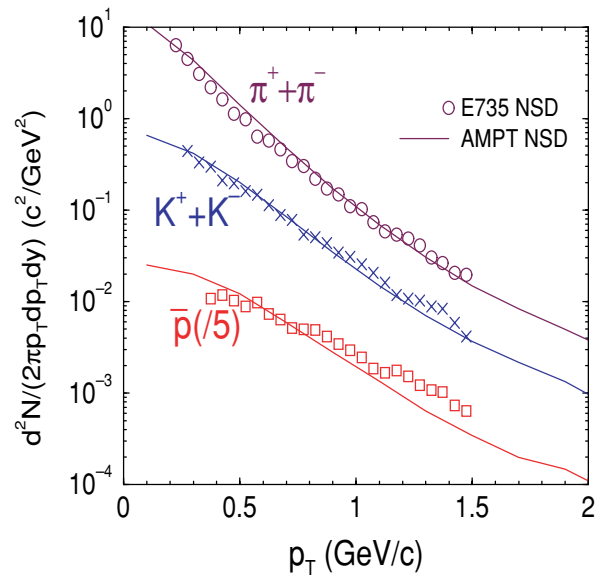

FIG. 12. (Color online) Transverse momentum spectra of charged pions, charged kaons, and antiprotons from $p \bar{p}$ collisions at $\sqrt{s}=$ $1.8 \mathrm{TeV}$.

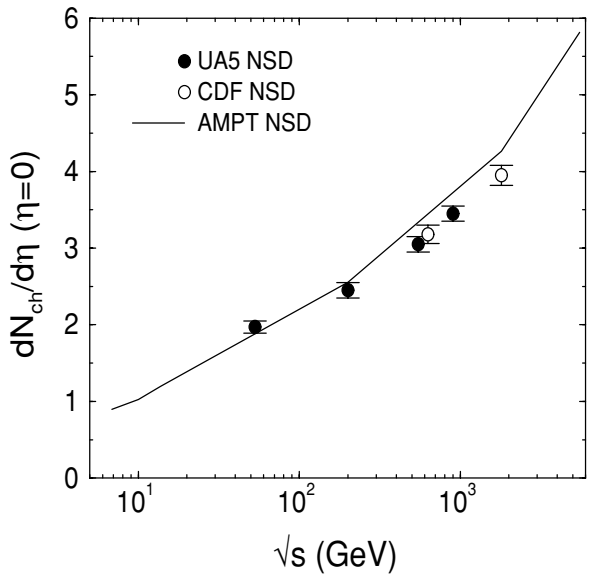

FIG. 13. Energy dependence of $d N_{\mathrm{ch}} / d \eta$ at midpseudorapidity for $p p$ and $p \bar{p}$ collisions.

AMPT model with these $a$ and $b$ parameters underpredicts both the negatively charged particle [39] and kaon multiplicities.

To increase the particle multiplicity in the AMPT model, we vary the $a$ and $b$ parameters, and find that the experimental data can be reasonably reproduced with $a=2.2$ and $b=$ $0.5 \mathrm{GeV}^{-2}$ as shown in Fig. 17. As seen from Eq. (9), a larger value of $a$ corresponds to a softer fragmentation function; i.e., it leads to a smaller average transverse momentum for produced hadrons and thus increases the particle multiplicity. The modified parameters also affect the string tension. According to Eq. (12), it is now 7\% larger, and this leads to a strangeness suppression factor of 0.33 instead of the default value of 0.30. Since the HIJING model with default $a$ and $b$ parameters reproduces the charged-particle multiplicities in $p p$ and $p \bar{p}$ collisions, the AMPT model with these parameters can probably describe peripheral heavy ion collisions. The $a$ and $b$ values in the AMPT model is thus expected to depend on the centrality of heavy ion collisions, but this has not been studied.

Since the probability for minijet production in the HIJING model is very small in collisions at SPS energies (only about four minijet partons for a central $\mathrm{Pb}+\mathrm{Pb}$ event on average),

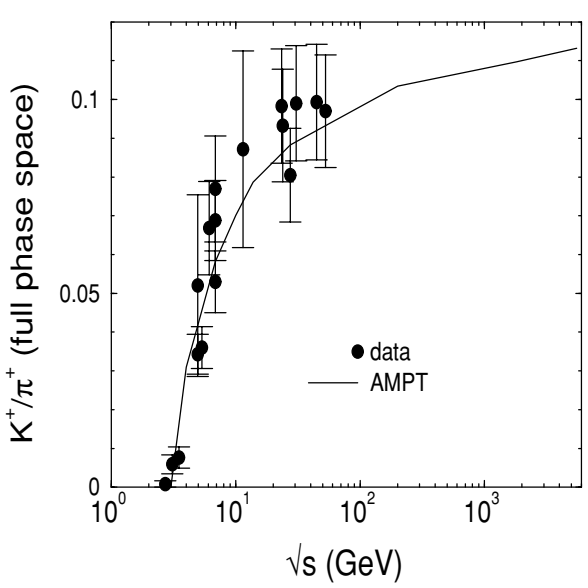

FIG. 14. Energy dependence of the $K^{+} / \pi^{+}$ratio in full phase space for $p p$ collisions. 


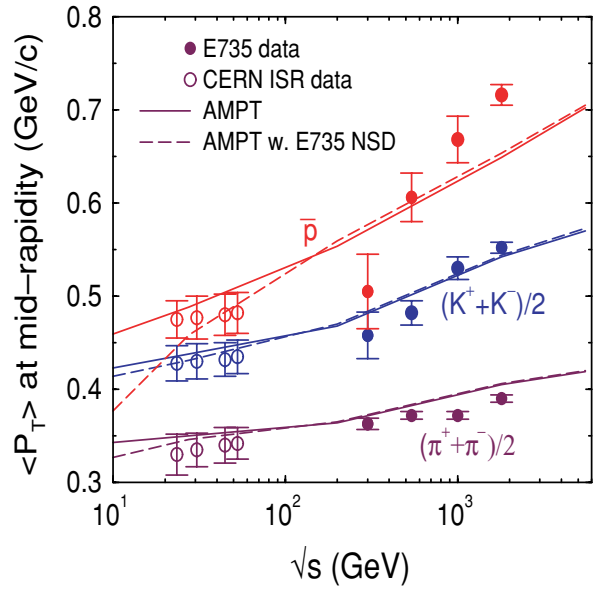

FIG. 15. (Color online) Energy dependence of the mean transverse momenta of pions, kaons, and antiprotons for $p p$ and $p \bar{p}$ collisions.

the partonic stage in the default AMPT model does not play any role for most observables in these collisions. Final-state hadronic scatterings are, however, important, and their effects are illustrated in Fig. 18. It is seen that final-state interactions reduce the pion and antiproton yields, but increase strangeness production; e.g., the sum of $K^{-}$and $\Lambda$ as well as the sum of $K^{+}$and $\bar{\Lambda}$ are both increased by about $20 \%$. Overall, the kaon yield in the default AMPT model is larger than that in the HIJING model by about $40 \%$ because of the combined effects of modified Lund string fragmentation and final-state interactions.
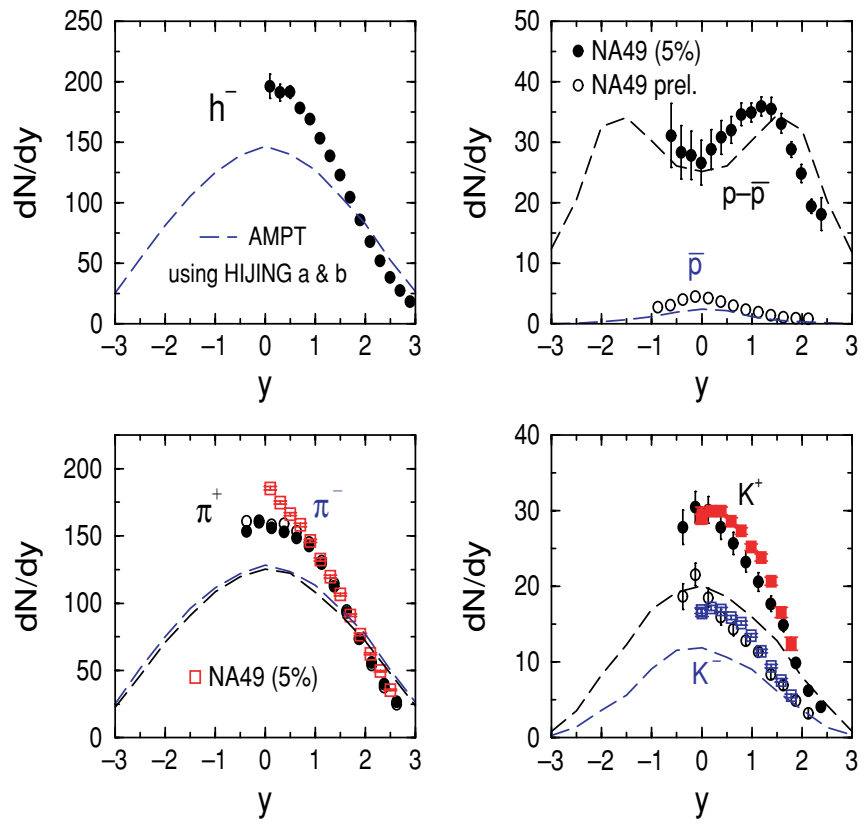

FIG. 16. (Color online) Rapidity distributions in central $(b \leqslant$ $3 \mathrm{fm}) \mathrm{Pb}+\mathrm{Pb}$ collisions at $\sqrt{s_{N N}}=17.3 \mathrm{GeV}$. Circles and squares are experimental data, while dashed curves are results from the AMPT model using default $a$ and $b$ parameters as in the HIJING model.
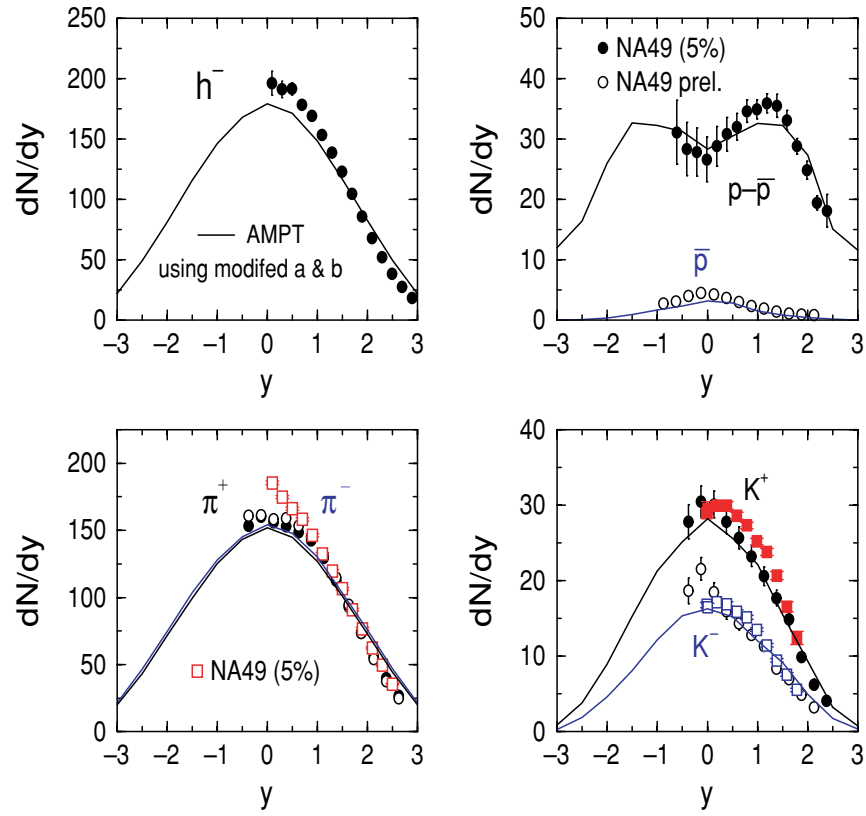

FIG. 17. (Color online) Same as Fig. 16, but with solid curves for results from the AMPT model using modified $a$ and $b$ parameters in the Lund string fragmentation function.

\section{B. Baryon stopping and antiproton production}

Understanding baryon stopping in relativistic heavy ion collisions is important because it is related to the total energy deposited in the produced hot dense matter during the collisions. The observed relatively large baryon stopping in these collisions has led to the novel suggestion of gluon junction transport in an initial excited baryon and its subsequent
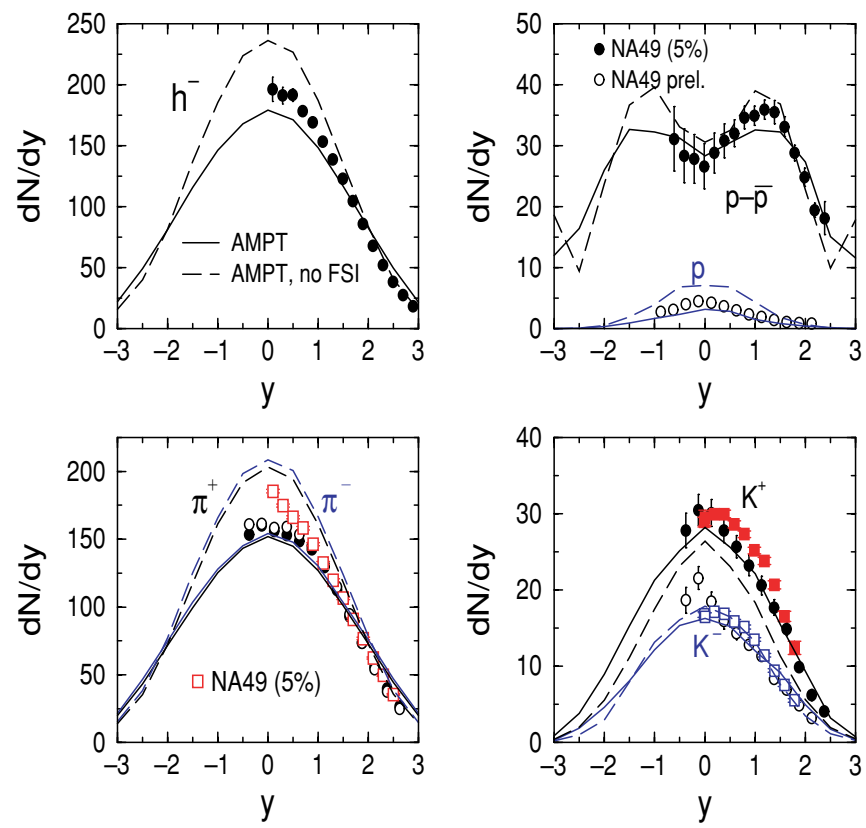

FIG. 18. (Color online) Same as Fig. 17, but with dashed curves for results from the AMPT model without partonic and hadronic finalstate interactions. 
decay into a slowly moving baryon and three leading beam mesons [56]. In the AMPT model, we have taken instead a more phenomenological approach to baryon stopping in relativistic heavy ion collisions by including the popcorn mechanism for baryon-antibaryon pair production from string fragmentation. The popcorn mechanism introduces two additional baryon production channels, i.e., the $B \bar{B}$ and $B M \bar{B}$ configurations in the Lund fragmentation model, which are controlled by two parameters in the JETSET/PYTHIA routine [59] used in the HIJING model. The first parameter MSTJ(12) is changed from 1 as set in the default HIJING model to 3 in the AMPT model $[40,42]$ in order to activate the popcorn mechanism, and the second parameter PARJ(5) controls the relative percentage of the $B \bar{B}$ and $B M \bar{B}$ channels. We find that with equal probabilities for the $B \bar{B}$ and $B M \bar{B}$ configurations, the net-baryon rapidity distribution at SPS can be reproduced as shown in Fig. 17 [42]. Without the popcorn mechanism, as in the default HIJING model, the net-baryon rapidity distribution would peak at a larger rapidity [39]. We also see from Fig. 18 that the antiproton yield at SPS is sensitive to the antibaryon annihilation and production channels discussed in Sec. IID 3. Without these reactions in the hadronic phase, the antiproton yield is too high compared to preliminary NA49 data.

\section{Transverse momentum spectra}

For the transverse momentum spectra, results from the default AMPT model are shown by solid curves in Fig. 19 for midrapidity pions, kaons, and protons in central $(b \leqslant 3 \mathrm{fm})$ $\mathrm{Pb}+\mathrm{Pb}$ collisions at the SPS energy of $\sqrt{s_{N N}}=17.3 \mathrm{GeV}$. Compared with experimental data from NA44, given by solid diamonds, the AMPT model gives a reasonable description up to transverse mass of about $1 \mathrm{GeV} / c^{2}$ above the particle mass. Without including rescatterings in the hadronic phase, the inverse slope parameters for the transverse mass spectra of kaons and protons from the AMPT model, given by dashed curves, are significantly reduced because of the absence of transverse flow that is induced by final-state interactions.

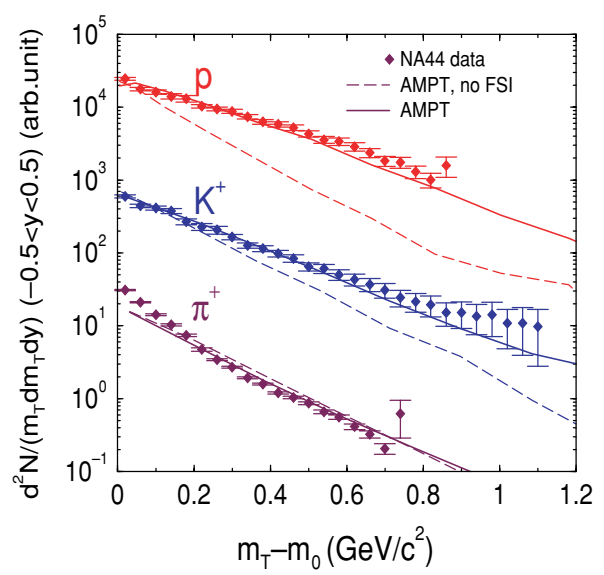

FIG. 19. (Color online) Transverse momentum spectra at midrapidity from the AMPT model with and without including hadron cascade compared with experimental data from the NA44 Collaboration for central $\mathrm{Pb}+\mathrm{Pb}$ collisions at SPS.

\section{RESULTS AT RHIC ENERGIES}

\section{A. Rapidity distributions}

Since the number of strings associated with soft interactions in the HIJING model depends weakly on the colliding energy, and the atomic number of $\mathrm{Au}$ is close to that of $\mathrm{Pb}$, we use the same modified parameters in the Lund string fragmentation model used for $\mathrm{Pb}+\mathrm{Pb}$ collisions at SPS energies for $\mathrm{Au}+\mathrm{Au}$ collisions at RHIC energies. In Fig. 20, results for central ( $b \leqslant 3 \mathrm{fm}) \mathrm{Au}+\mathrm{Au}$ collisions at center-of-mass energies of $\sqrt{s_{N N}}=130 \mathrm{GeV}$ (dashed curves) and $200 \mathrm{GeV}$ (solid curves) are shown together with data from the PHOBOS Collaboration [110-112] and the BRAHMS Collaboration [113]. We find that measured total charged-particle pseudorapidity distributions at both energies are roughly reproduced. More detailed comparisons on pseudorapidity distributions at different centralities and different RHIC energies have been carried out by the BRAHMS Collaboration [114,115], where results from the default AMPT model are compatible with the data. However, compared to central (top 5\%) BRAHMS data, the AMPT model tends to overpredict the height of the rapidity distributions of charged pions, kaons, protons, and antiprotons. Note that the BRAHMS data on protons and antiprotons at $y=0$ in Fig. 20 have been corrected for feed-down from weak decays.

Other models have also been used to study hadron rapidity distributions at RHIC. While results from the HIJING model $[65,116]$ are compatible with the observed charged-particle rapidity density, the model does not include interactions among minijet partons and final-state interactions among hadrons. The saturation model without final-state interactions also reproduces the experimental data [32,34]. Furthermore, the hadronic cascade model LUCIFER [22] predicts a
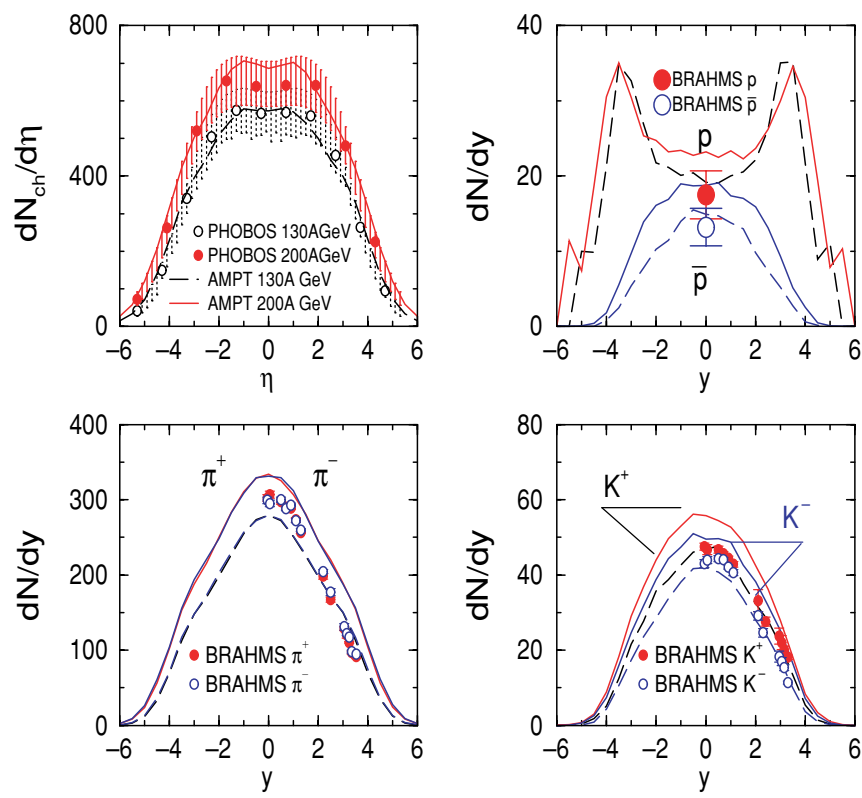

FIG. 20. (Color online) Rapidity distributions for $\mathrm{Au}+\mathrm{Au}$ collisions at $\sqrt{s_{N N}}=130$ and $200 \mathrm{GeV}$. Circles are the PHOBOS data for the $6 \%$ most central collisions or the BRAHMS data for the $5 \%$ most central collisions at $\sqrt{s_{N N}}=200 \mathrm{GeV}$, and curves are results from the default AMPT model for $b \leqslant 3 \mathrm{fm}$. 

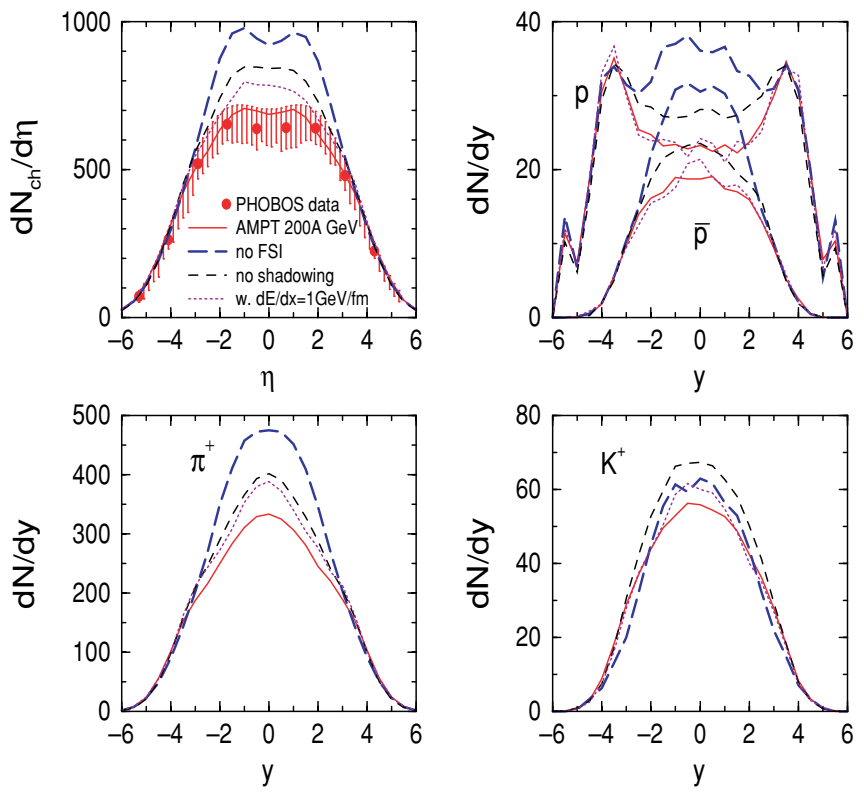

FIG. 21. (Color online) Rapidity distributions of charged particles in central $(b \leqslant 3 \mathrm{fm}) \mathrm{Au}+\mathrm{Au}$ collisions at $\sqrt{s_{N N}}=$ $200 \mathrm{GeV}$ from the default AMPT model (solid curves), without finalstate interactions (long-dashed curves), without nuclear shadowing (dashed curves), and with jet quenching of $d E / d x=-1 \mathrm{GeV} / \mathrm{fm}$ (dotted curves).

charged-particle multiplicity at midrapidity that is comparable to the RHIC data [24]. On the other hand, the LEXUS model [17], which is based on a linear extrapolation of ultrarelativistic nucleon-nucleon scattering to nucleus-nucleus collisions, predicts too many charged particles [117] compared with the PHOBOS data. The URQMD model [19] also failed [114] in describing the charged-particle multiplicity at RHIC.

To see the effect of hadronic interactions, we show in Fig. 21 by long-dashed curves the rapidity distributions of charged particles obtained from the default AMPT model without finalstate interactions (i.e., without parton and hadron cascades) for central $(b \leqslant 3 \mathrm{fm}) \mathrm{Au}+\mathrm{Au}$ collisions at $\sqrt{s_{N N}}=200 \mathrm{GeV}$. Compared to the case with hadronic scatterings, there is a significant increase in the numbers of total charged particles and pions at midrapidity. The kaon number, on the other hand, only increases slightly when production from final-state hadronic interactions is excluded. The ratios of $\bar{p} / p$ and $K^{+} / \pi^{+}$at midrapidity are 0.85 and 0.13 , respectively, in the absence of final-state interactions, instead of 0.81 and 0.17 from the default AMPT model including the hadron cascade. We note that although the default HIJING $[65,116]$ with original $a$ and $b$ parameters gives a total charged-particle multiplicity at midrapidity that is consistent with the RHIC data, the inclusion of hadronic scatterings reduces appreciably the final number.

We also find that excluding parton cascade in the AMPT model changes the final charged-particle yield at midrapidity at $\sqrt{s_{N N}}=200 \mathrm{GeV}$ by less than $5 \%$. This indicates that hadron yields are not very sensitive to parton elastic scatterings in the default AMPT model. To take into account the effect of parton inelastic collisions such as gluon radiation, which is not included in ZPC as it includes at present only elastic scatterings, we have included in the AMPT model the default HIJING jet quenching, i.e., an energy loss of $d E / d x=$ $-1 \mathrm{GeV} / \mathrm{fm}$, before minijet partons enter the ZPC parton cascade. Results obtained with jet quenching for central $\mathrm{Au}+\mathrm{Au}$ collisions at $\sqrt{s_{N N}}=200 \mathrm{GeV}$ are shown in Fig. 21 by dotted curves. We see that the quenching effects are larger for pions than for kaons, protons, and antiprotons. Since present calculations from the AMPT model without jet quenching already reproduce the data at collision energy of $\sqrt{s_{N N}}=$ $200 \mathrm{GeV}$, and further inclusion of jet quenching of $d E / d x=$ $-1 \mathrm{GeV} / \mathrm{fm}$ increases the final yield of total charged particles at midrapidity by about $14 \%$, our results for the rapidity distribution of charged particles are thus consistent with a weak jet quenching at this energy. However, the dense matter created in heavy ion collisions expands rapidly, thus the energy loss at the early stage may still be large [118,119].

The effect of initial nuclear shadowing of the parton distributions in nuclei is also shown in Fig. 21. Without the initial nuclear shadowing effect on minijet parton production, the charged-particle multiplicity at midrapidity in central $\mathrm{Au}+\mathrm{Au}$ collisions at $\sqrt{s_{N N}}=200 \mathrm{GeV}$ increases by about $23 \%$. Although this increase can be offset by using different parameters in the Lund string fragmentation, to reproduce both SPS and RHIC data with the same parameters in the default AMPT model requires the inclusion of nuclear shadowing on minijet parton production.

\section{B. Particle ratios}

The energy dependence of the yields of particles and their ratios at midrapidity from central $\mathrm{Au}+\mathrm{Au}$ collisions at AGS to central $\mathrm{Au}+\mathrm{Au}$ collisions at RHIC energies are shown in Fig. 22. Curves represent AMPT results, while

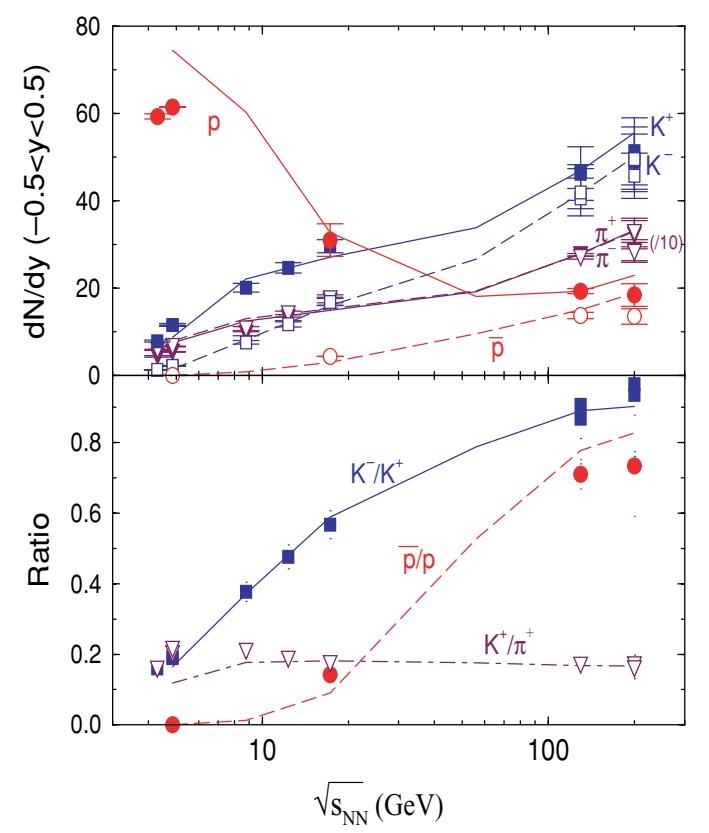

FIG. 22. (Color online) Energy dependence of particle yields and ratios of $K^{-} / K^{+}, \bar{p} / p$, and $K^{+} / \pi^{+}$at midrapidity in central $\mathrm{Au}+\mathrm{Au}$ or $\mathrm{Pb}+\mathrm{Pb}$ collisions. 
data from AGS, SPS, and RHIC experiments are shown by symbols: Triangles for pions, squares for kaons, and circles for protons and antiprotons in the upper figure. The AGS data are from the E917 and E866 Collaborations for central $\mathrm{Au}+\mathrm{Au}$ collisions at laboratory kinetic energies of 7.94 and 10.7 GeV per nucleon [120], from the E895 Collaboration for central $\mathrm{Au}+\mathrm{Au}$ collisions at the laboratory kinetic energy of $8 \mathrm{GeV}$ per nucleon [121], and from the E802 Collaboration for central $\mathrm{Au}+\mathrm{Au}$ collisions at $P_{\text {lab }}=11.6-11.7 \mathrm{GeV} / c$ per nucleon [122]. The SPS data are from NA49 for central $\mathrm{Pb}+\mathrm{Pb}$ collisions at laboratory energies of $40 A, 80 A$, and $158 A \mathrm{GeV}$ [105]. The RHIC data are from the PHENIX Collaboration $[123,124]$ and the STAR Collaboration [125,126] for central $\mathrm{Au}+\mathrm{Au}$ collisions at $\sqrt{s_{N N}}=130$ and $200 \mathrm{GeV}$.

Since the antiproton yield increases with energy almost logarithmically while the proton yield initially decreases at low energies, the $\bar{p} / p$ ratio (data represented by circles) increases rapidly from almost 0 at AGS to about 0.1 at the SPS energy of $158 A \mathrm{GeV}$ then rapidly to about 0.8 at the maximum RHIC energy of $\sqrt{s_{N N}}=200 \mathrm{GeV}$, indicating the formation of a nearly baryon-antibaryon symmetric matter at RHIC. The $K^{+} / \pi^{+}$ratio (data represented by triangles), on the other hand, is almost constant from the SPS energy of $158 \mathrm{~A} \mathrm{GeV}$ to RHIC, suggesting an approximate chemical equilibrium of strangeness in heavy ion collisions in this energy range [127]. The $K^{-} / K^{+}$ratio (data represented by squares) increases from below 0.2 at AGS to about 0.6 at the SPS energy of $158 A \mathrm{GeV}$, then gradually to about 0.9 at $\sqrt{s_{N N}}=200 \mathrm{GeV}$, and the value near 1 at the top RHIC energy is closely related to the fact that the matter formed at RHIC is nearly baryon-antibaryon symmetric.

\section{Baryon and antibaryon production}

Figure 23 shows the net-proton and antiproton rapidity distributions from the default AMPT model at two different

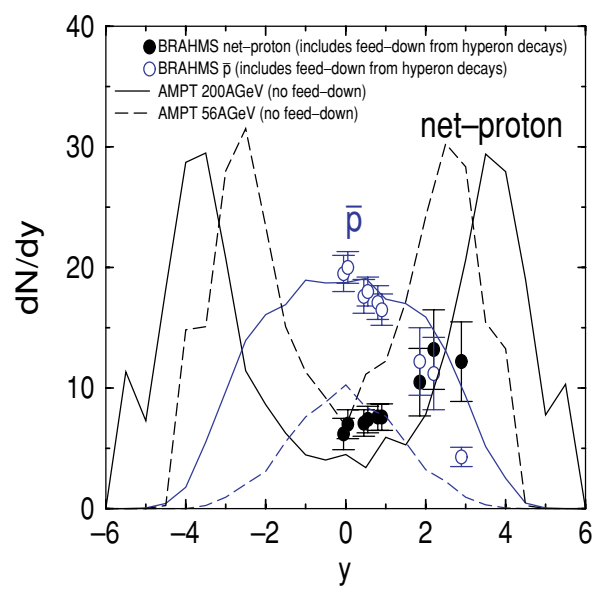

FIG. 23. (Color online) Net-proton and antiproton rapidity distributions from the default AMPT model for central $(b \leqslant 3 \mathrm{fm}) \mathrm{Au}+\mathrm{Au}$ collisions at RHIC energies of $\sqrt{s_{N N}}=200 \mathrm{GeV}$ (solid curves) and $56 \mathrm{GeV}$ (dashed curves) VS data from the BRAHMS Collaboration for $5 \%$ most central collisions at $\sqrt{s_{N N}}=200 \mathrm{GeV}$.
RHIC energies. Filled and open circles are BRAHMS data on net-proton and antiprotons without taking into account corrections due to weak decay of hyperons [128], i.e., the data include feed-down from hyperon weak decays. Since the AMPT results on proton and antiprotons exclude feed-down from weak decays, the antiproton yield at midrapidity is actually over-predicted by the AMPT model, as can be more clearly seen in Fig. 20; while the net-proton rapidity distributions may agree reasonably with the BRAHMS data.

It is further seen that the antiproton yield at midrapidity increases rapidly with collision energy, and peaks of net-proton and proton distributions shift toward larger rapidity at higher collision energies. Since the proton yield at midrapidity first decreases from the AGS energy to the RHIC energy of $\sqrt{s_{N N}}=56 \mathrm{GeV}$ and eventually increases slowly with energy (see Fig. 22), it may have a minimum between the SPS energy and the maximum RHIC energy. On the other hand, the net-proton yield at midrapidity decreases with the colliding energy in the energy range from the AGS energy to the maximum RHIC energy.

\section{Transverse momentum spectra}

For transverse momentum spectra, results from the default AMPT model for pions, kaons, and protons at midrapidity from central $(b \leqslant 3 \mathrm{fm}) \mathrm{Au}+\mathrm{Au}$ collisions at $\sqrt{s_{N N}}=200 \mathrm{GeV}$ are shown in Fig. 24 together with the 5\% most central data from the PHENIX Collaboration [123]. Below $p_{\mathrm{T}}=2 \mathrm{GeV} / c$, the default AMPT model gives a reasonable description of the pion and kaon spectra [43]. It overpredicts, however, both the proton and antiproton spectra at low $p_{\mathrm{T}}$. We note that the PHENIX data have been corrected for weak decays.

The effect due to final-state hadronic scatterings on pion and proton transverse momentum spectra is illustrated in Fig. 25, where solid and dashed curves with statistical errors are, respectively, the results with and without hadron cascade in the default AMPT model. As found in Fig. 19 at SPS energies,

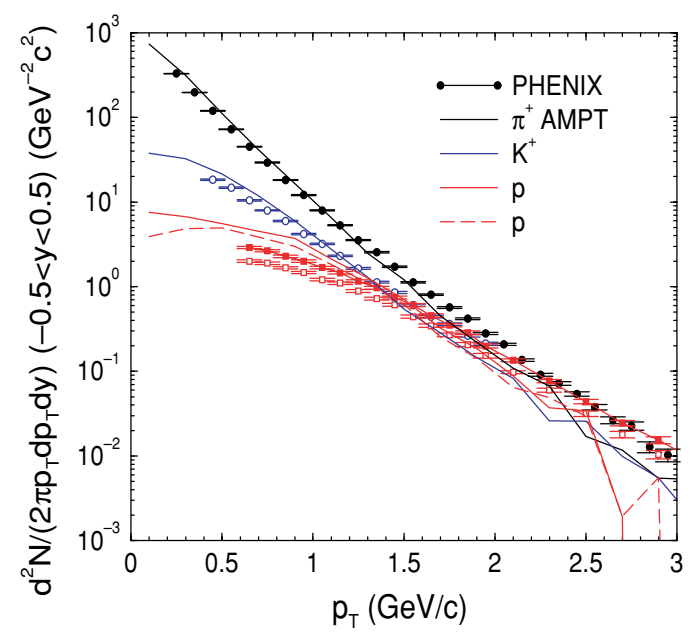

FIG. 24. (Color online) Transverse momentum spectra of midrapidity pions, kaons, protons, and antiprotons from central $(b \leqslant 3 \mathrm{fm}) \mathrm{Au}+\mathrm{Au}$ collisions at $\sqrt{s_{N N}}=200 \mathrm{GeV}$ from the default AMPT model. Data are from the PHENIX Collaboration. 


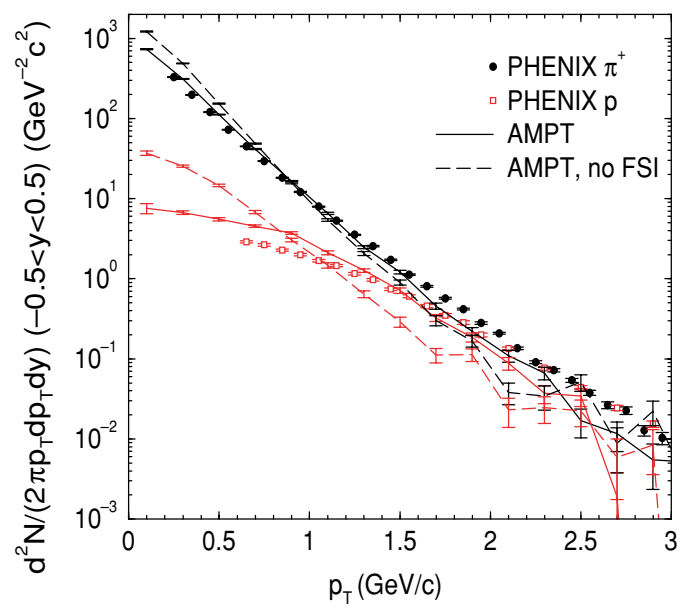

FIG. 25. (Color online) Transverse momentum spectra of midrapidity pions and protons from the default AMPT model with (solid curves) and without (dashed curves) final-state interactions in central $\mathrm{Au}+\mathrm{Au}$ collisions at $\sqrt{s_{N N}}=200 \mathrm{GeV}$.

hadronic rescatterings increase significantly the inverse slope of the proton transverse momentum spectrum while they do not affect much that of pions. As a result, the final proton yield at midrapidity becomes close to the pion yield at $p_{\mathrm{T}} \sim 2 \mathrm{GeV} / c$, as observed in experiments at RHIC [123,129]. Without final-state scatterings, the proton yield given by the dashed curve is well below the pion yield up to $p_{\mathrm{T}} \sim$ $1.7 \mathrm{GeV} / c$ when statistical fluctuations in the AMPT calculations become large. Results from the default AMPT model thus indicate that the observed large $p / \pi$ ratio at $p_{\mathrm{T}} \sim 2 \mathrm{GeV} / c$ is due to the collective transverse flow generated by final-state rescatterings.

The above results are obtained without quenching of minijet partons due to gluon radiations. Including this effect would reduce the yield of hadrons at large transverse momentum as shown in Fig. 26, leading to an appreciable discrepancy

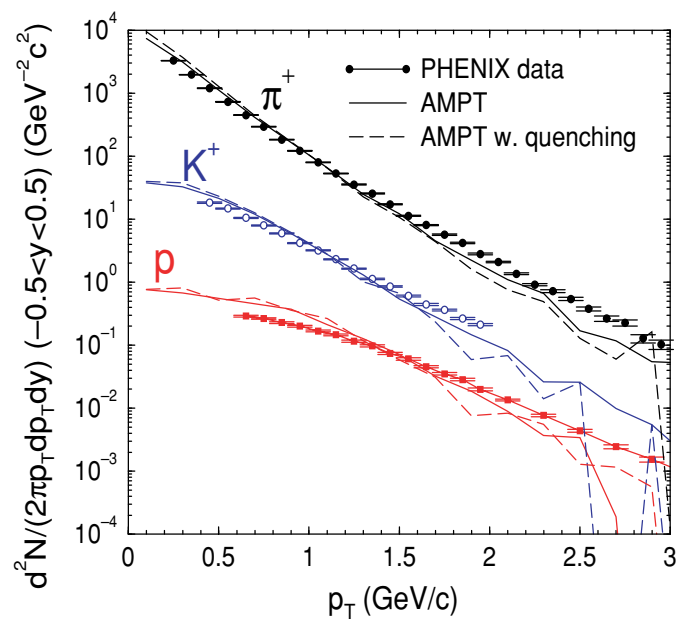

FIG. 26. (Color online) Transverse momentum spectra with and without quenching of $d E / d x=-1 \mathrm{GeV} / \mathrm{fm}$ for central $\mathrm{Au}+\mathrm{Au}$ collisions at $\sqrt{s_{N N}}=200 \mathrm{GeV}$ from the default AMPT model vs data from the PHENIX Collaboration.

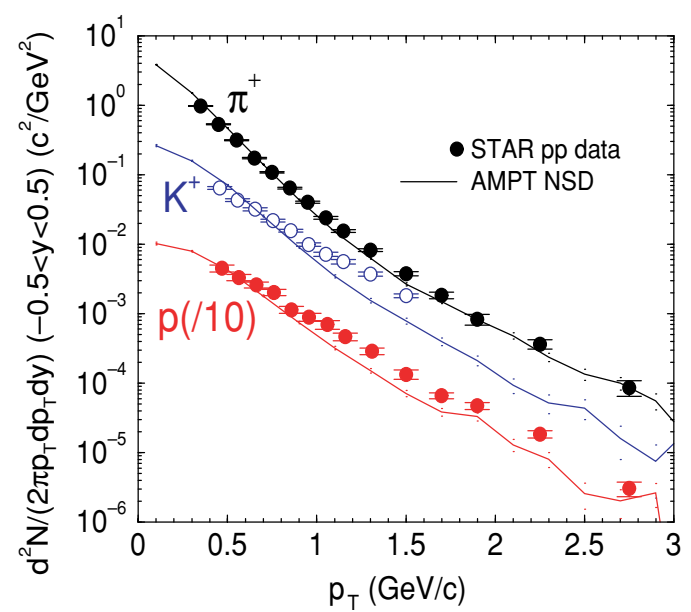

FIG. 27. (Color online) Transverse momentum spectra of midrapidity pions, kaons, and protons for $p p$ collisions at $200 \mathrm{GeV}$ from the default AMPT model vs data from the STAR Collaboration.

between the AMPT results and the experimental data from the PHENIX Collaboration for hadrons with momenta greater than $1.5 \mathrm{GeV} / c$. This discrepancy may stem, however, from the fact that the AMPT model already underpredicts the STAR data for hadron yield above $1 \mathrm{GeV} / c$ in $p p$ collisions as shown in Fig. 27. Since the STAR results are consistent with previous UA5/CDF measurements at similar multiplicities [126] but have significantly higher mean kaon and proton transverse momenta than interpolated values from the UA5/CDF $p p / p \bar{p}$ data shown in Fig. 15, the STAR NSD trigger might be quite different from the UA5/CDF NSD trigger. To make a more definitive conclusion on jet quenching in $\mathrm{Au}+\mathrm{Au}$ collisions thus requires a focused and higher-statistics study of $p_{\text {T }}$ spectra in both $p p$ and $\mathrm{Au}+\mathrm{Au}$ collisions.

\section{E. $\phi$ meson yield via dilepton and dikaon spectra}

Since the $\phi$ meson is unstable, it can only be detected from its decay products such as the kaon-antikaon pair or the lepton pair. In the AMPT model, we follow the production and decay of $\phi$ mesons as well as the scattering of the kaon daughters from their decays. Since the latter destroys the possibility of reconstructing the parent $\phi$ mesons, only $\phi$ mesons with their decay kaons not undergoing scattering can be reconstructed from the kaon-antikaon invariant mass distribution. The kaon-antikaon pair from a $\phi$ meson decay is likely to undergo rescatterings in the medium, which would lead to a reconstructed invariant mass outside the original $\phi$ meson peak. Hence $\phi$ mesons decaying in the dense medium are difficult to be identified via reconstructed kaon-antikaon pairs. In contrast, dileptons have negligible final-state interactions with the surrounding hadronic medium, they are thus considered to carry useful information about hadron properties in hot dense hadronic matter [130,131], which are expected to be different from those in free space $[132,133]$. 
Since dimuons are emitted continuously during evolution of the system, the total number of dimuons is given by

$$
N_{\mu^{+} \mu^{-}}=\int_{0}^{t_{\mathrm{cut}}} d t N_{\phi}(t) \Gamma_{\phi \rightarrow \mu^{+} \mu^{-}}(M)+N_{\phi}\left(t_{\mathrm{cut}}\right) \frac{\Gamma_{\phi \rightarrow \mu^{+} \mu^{-}}}{\Gamma_{\phi}},
$$

where $N_{\phi}(t)$ denotes the number of $\phi$ mesons at time $t$ and $\Gamma_{\phi \rightarrow \mu^{+} \mu^{-}}(M)$ is its decay width to dimuon as given by

$$
\Gamma_{\phi \rightarrow \mu^{+} \mu^{-}}(M)=C_{\mu^{+} \mu^{-}} \frac{m_{\phi}^{4}}{M^{3}}\left(1-\frac{4 m_{\mu}^{2}}{M^{2}}\right)^{1 / 2}\left(1+\frac{2 m_{\mu}^{2}}{M^{2}}\right) .
$$

The coefficient $C_{\mu^{+} \mu^{-}} \equiv \alpha^{2} / 27\left(g_{\phi K \bar{K}}^{2} / 4 \pi\right)=1.634 \times 10^{-6}$ is determined from measured width of free $\phi$ meson at invariant mass $M=m_{\phi}$. In Eq. (17), the first term on the right-hand side corresponds to dimuon production before a cutoff time $t_{\text {cut }}$ during the hadronic phase of heavy ion collisions, while the second term refers to dimuon emission after that cutoff time. The reconstructed $\phi$ meson number is then obtained by dividing the above expression by the dimuon branching ratio in free space of $\Gamma_{\phi \rightarrow \mu^{+} \mu^{-}} / \Gamma_{\phi}=3.7 \times 10^{-4}$. The number of kaon-antikaon pairs coming from $\phi$ meson decays can be similarly expressed as Eq. (17):

$$
N_{K \bar{K}}=\int_{0}^{t_{\mathrm{cut}}} d t N_{\phi}(t) \Gamma_{\phi \rightarrow K \bar{K}}(M)+N_{\phi}\left(t_{\mathrm{cut}}\right) \frac{\Gamma_{\phi \rightarrow K \bar{K}}}{\Gamma_{\phi}} .
$$

The $\phi$ meson number from kaon-antikaon decays is then obtained by dividing Eq. (19) by the $K \bar{K}$ branching ratio in free space $\Gamma_{\phi \rightarrow K \bar{K}} / \Gamma_{\phi}$.

Using the default AMPT model, we studied the $\phi$ meson yield reconstructed from $K^{+} K^{-}$and $\mu^{+} \mu^{-}$pairs for central $\mathrm{Pb}+\mathrm{Pb}$ collisions at SPS and for central $\mathrm{Au}+\mathrm{Au}$ collisions at RHIC [47]. In both cases, we found that due to hadronic rescattering and absorption in the kaonic channel, the $\phi$ meson abundance at midrapidity is larger in the dimuon channel than in the dikaon channel, and the inverse slope parameter obtained from the transverse mass spectra of $\phi$ mesons in the range $0<$ $m_{T}-m_{\phi}<1 \mathrm{GeV} / c^{2}$ is larger in the dimuon channel than in the dikaon channel. These features are consistent with the data at SPS from the NA49 [134] and NA50 [135] Collaborations. Comparison of the results for RHIC with future experimental data will allow us to learn if enhanced $\phi$ meson production is due to the formation of the quark-gluon plasma during the early stage of collisions [47].

\section{F. Multistrange baryon production}

One possible signal for the quark-gluon plasma is enhanced production of strange particles [136], particularly those consisting of multistrange quarks such as $\Xi$ and $\Omega$ baryons as well as their antiparticles. The argument is that the rate for strange hadron production is small in hadronic matter on account of large threshold and small cross sections, while the production rate for strange quarks is large in the quark-gluon plasma [87].

A detailed study of multistrange baryons in the AMPT model [44] shows that although few multistrange baryons are produced in HIJING (see Table I of Ref. [44]), including the strangeness-exchange interactions listed in Sec. IID 4 in the default AMPT model leads to an enhanced production of multistrange baryons in heavy ion collisions at both SPS and RHIC. For central $\mathrm{Pb}+\mathrm{Pb}$ collisions at SPS, the strangenessexchange reactions enhance modestly the yields of $\Lambda, \Sigma$, and $\Xi$, but increase the $\Omega$ yield by more than an order of magnitude. However, the $\Omega$ yield in central $\mathrm{Pb}+\mathrm{Pb}$ collisions at SPS from the default AMPT model is still about a factor of 2 lower than the data, and this may indicate that strangeness production is already enhanced during the early stage of collisions.

Predictions from the default AMPT model [44] also show that the slope parameters obtained from the transverse mass spectra of multistrange baryons reveal a plateau structure since these particles, mostly generated by strangeness-exchange reactions in the model, are weakly interacting and decouple rather early from the system. It will be very interesting to test these predictions against the RHIC data. In particular, the quark coalescence model [137-145], which assumes that all energy in the early stage of RHIC collisions is in the partonic degrees of freedom as assumed in the string melting scenario $[45,48,50]$ of the AMPT model, relates the transverse momentum spectra or the elliptic flow of multistrange baryons with other hadrons such as kaons and protons. These relations can be drastically different from the default AMPT model, where the partonic stage includes only minijet partons and has a much smaller effect than the later hadronic stage.

\section{G. Equilibration between $J / \psi$ and open charm}

$J / \psi$ production has long been proposed as a possible signal for the formation of the quark-gluon plasma in relativistic heavy ion collisions [146]. Based on the expectation that the effective potential between charm and anticharm quarks changes in the QGP because of the color screening effect, they will not form bound states above a certain critical temperature, which is somewhat higher than the deconfinement temperature. The qualitative change in the heavy quark effective potential has been verified by results from lattice QCD simulations [147-149]. As a result, $J / \psi$ production is expected to be suppressed if the QGP is formed in heavy ion collisions. In particular, the observed abnormal suppression of $J / \psi$ in central $\mathrm{Pb}+\mathrm{Pb}$ collisions at SPS has been attributed to the formation of the quark-gluon plasma in these collisions $[150,151]$. However, there are other possible mechanisms for $J / \psi$ suppression in heavy ion collisions [152-154], e.g., $J / \psi$ may be destroyed by collisions with incoming nucleons or with gluons in the initial partonic matter $[155,156]$ or with comoving hadrons in the hadronic matter [157-171].

For heavy ion collisions at RHIC energies, multiple pairs of charm-anticharm quarks can be produced in one event. Estimates using the kinetic approach [172] have shown that the number of $J / \psi$ produced from the interaction between charm and anticharm quarks, i.e., the inverse reaction of $J / \psi$ dissociation by gluons, may exceed that expected from the superposition of initial nucleon-nucleon interactions. Calculations based on the statistical model [173-175], which 
assumes that $J / \psi$ formed during hadronization of the QGP is in chemical equilibrium with charm mesons, also predict that the $J / \psi$ number is comparable to the expected primary yield.

To study the above-discussed effects on $J / \psi$ production, the default AMPT model has been modified [176] to include $J / \psi$ absorption and production in both initial partonic and final hadronic matters [41]. It was found that because of these final-state interactions, the final $J / \psi$ yield in central $\mathrm{Au}+\mathrm{Au}$ collisions at RHIC may exceed the suppressed primary $J / \psi$ yield which is based only on the color screening mechanism. Furthermore, the $J / \psi$ yield at RHIC depends on the charm quark mass in the partonic matter and charmed hadron masses in the hadronic matter.

We note that the elliptic flows of $J / \psi$ and charmed hadrons are related in the quark coalescence model, and they exhibit novel mass effects when the constituent quark masses in the hadron are different [142]. The transverse momentum spectra of electrons from open charm decays are, however, insensitive to the charm transverse flow [177]. Comparing RHIC data on charm hadron or electron $v_{2}$ [143] with results from the quark coalescence model may allow us to determine whether in heavy ion collisions, charm quarks flow collectively as light quarks.

\section{H. Elliptic flow}

Elliptic flow in heavy ion collisions is a measure of the asymmetry of particle momentum distributions in the transverse plane and is generated by the anisotropic pressure gradient in initial hot dense matter as a result of the spatial asymmetry in noncentral collisions [178-183]. It is defined as one half of the second Fourier coefficient of the azimuthal angle distribution of particle transverse momentum and can be evaluated as

$$
v_{2}=\left\langle\frac{p_{x}^{2}-p_{y}^{2}}{p_{x}^{2}+p_{y}^{2}}\right\rangle .
$$

In the above, the $x$ axis is along the impact parameter in the transverse plane of each event, while the $y$ axis points out of the reaction plane, and the average is taken over the particles in consideration.

At RHIC, large hadron elliptic flows have been observed [184-190], and their dependence on transverse momentum $[184,185]$ and pseudorapidity $[186,191]$ as well as on particle species [187,192] have also been studied. To understand these experimental results, many theoretical models have been introduced, and these include semianalytic models [193], models based on parton energy loss [194,195], hydrodynamic models [9,180,196,197], transport models [45,181,198-201], including a hybrid model that uses the transport model as an afterburner of the hydrodynamic model [202], and quark coalescence models [137-145]. Transport models based on hadronic and/or string degrees of freedom in general give a smaller elliptic flow [187] than that observed at RHIC. Although hydrodynamic models can explain the large elliptic flow at low transverse momenta with a large initial energy density, they predict a continuously increasing elliptic flow with increasing hadron transverse momenta instead of the level

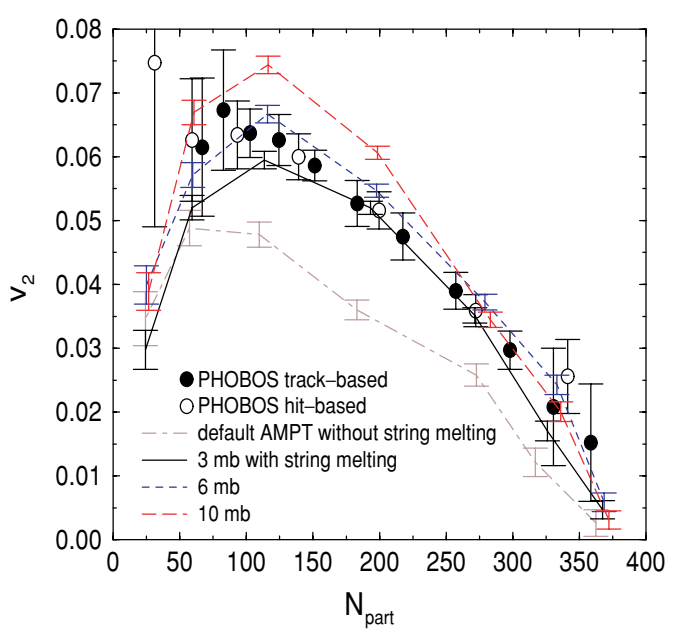

FIG. 28. (Color online) Centrality dependence of charged hadron elliptic flow in $\mathrm{Au}+\mathrm{Au}$ collisions at $\sqrt{s_{N N}}=200 \mathrm{GeV}$. Data from the PHOBOS Collaboration [205] are shown by circles; results from the AMPT mode with different partonic dynamics are shown by curves.

off observed in experiments [187,189,203,204], indicating that high-transverse-momentum particles do not reach thermal equilibrium. With the AMPT model, we can address such nonequilibrium effects to obtain information on the degree of thermalization in these collisions. It further allows us to study the effects of both partonic and hadronic rescatterings.

In Fig. 28, we show the elliptic flow of all charged particles in $\mathrm{Au}+\mathrm{Au}$ collisions at $\sqrt{s_{N N}}=200 \mathrm{GeV}$ from both the default AMPT model and the extended model with string melting as a function of $N_{\text {part }}$, which is the total number of participant nucleons after primary collisions but before partonic and hadronic rescatterings [49]. To compare with PHOBOS data [205], we included only charged particles with pseudorapidities and transverse momenta in the ranges $\eta \in$ $(-1,1)$ and $p_{\mathrm{T}} \in(0.1,4) \mathrm{GeV} / c$, respectively, for evaluating the elliptic flow. Error bars in the figure represent the statistical error in our calculations. We see that the value of $v_{2}$ depends not only on whether initial strings are converted to partons but also on the parton cross section used in the model, similar to that seen in heavy ion collisions at $\sqrt{s_{N N}}=$ $130 \mathrm{GeV}$ [45]. With the default AMPT model, which includes only scattering among minijet partons, the elliptic flow obtained with a parton scattering cross section of $3 \mathrm{mb}$ is found to be too small to account for the experimental data from the PHOBOS Collaboration. The elliptic flow is larger in the string melting scenario as soft partons from melted strings also participate in partonic scatterings, and its value is further increased when the parton scattering cross section increases from 3 to $10 \mathrm{mb}$, indicating that the dense system created in heavy ion collisions at RHIC does not quite reach thermal equilibrium.

Figure 29 shows the differential elliptic flows $v_{2}\left(p_{\mathrm{T}}\right)$ of pions, kaons, and protons from the AMPT model in the string melting scenario with a parton scattering cross section of $6 \mathrm{mb}$ for minimum-bias $\mathrm{Au}+\mathrm{Au}$ collisions at $\sqrt{s_{N N}}=$ $200 \mathrm{GeV}$. Although the PHENIX data are for particles with pseudorapidities $\eta \in(-0.35,0.35)$, the AMPT results are for 


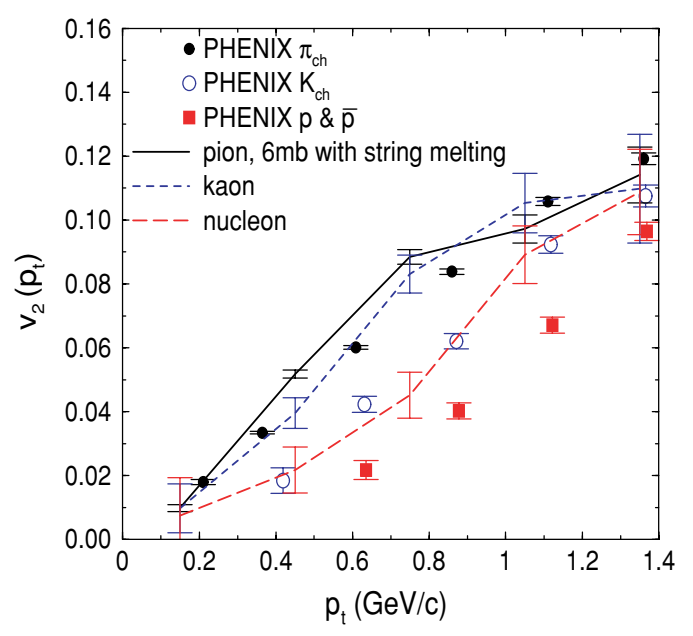

FIG. 29. (Color online) Transverse momentum dependence of elliptic flow of identified hadrons for minimum-bias $\mathrm{Au}+\mathrm{Au}$ collisions at $\sqrt{s_{N N}}=200 \mathrm{GeV}$. Circles are data from the PHENIX Collaboration [208] while curves are results from the AMPT model with string melting and using $6 \mathrm{mb}$ for the parton scattering cross section.

$\eta \in(-1,1)$ in order to improve the statistics of our calculation. In this low $p_{\mathrm{T}}$ region, we observe the mass ordering of $v_{2}\left(p_{\mathrm{T}}\right)$, i.e., hadrons with larger masses have smaller $v_{2}$ values at the same $p_{\mathrm{T}}$, similar to that from the hydrodynamic model $[8,9]$. For hadrons at higher $p_{\mathrm{T}}$, which are not studied here, a scaling of hadron elliptic flows according to their constituent quark content has been proposed based on the quark coalescence model [137-139,206]. Except for pions, this scaling seems to be confirmed by experimental data from both STAR [207] and PHENIX [208] Collaborations. The violation of pion elliptic flow from the constituent quark number scaling has been attributed to effects of resonance decays [209,210] and the binding energy of hadrons [142].

The pseudorapidity dependence of the elliptic flow in minimum-bias $\mathrm{Au}+\mathrm{Au}$ collisions at $\sqrt{s_{N N}}=200 \mathrm{GeV}$ has also been studied in the AMPT model [211]. While the string melting scenario with a parton scattering cross section of $3 \mathrm{mb}$ as well as the default AMPT model gives a better description of the PHOBOS data [205] at large pseudorapidity, the string melting scenario with a larger parton scattering cross section reproduces the PHOBOS data at midpseudorapidity. This may not be unreasonable as not all strings are expected to melt at large pseudorapidity where the smaller particle multiplicity leads to a lower energy density.

The AMPT model has further been used to study higher-order anisotropic flows $v_{4}$ and $v_{6}$ of charged hadrons at midrapidity in heavy ion collisions at RHIC [212]. It was found that the same large parton scattering cross section used in explaining the measured $v_{2}$ of charged hadrons could also reproduce recent data on their $v_{4}$ and $v_{6}$ from $\mathrm{Au}+\mathrm{Au}$ collisions at $\sqrt{s_{N N}}=200 \mathrm{GeV}$ [213]. Furthermore, $v_{4}$ was seen to be a more sensitive probe of the initial partonic dynamics in these collisions than $v_{2}$. Moreover, higher-order parton anisotropic flows are nonnegligible and satisfy the scaling relation $v_{n, q}\left(p_{\mathrm{T}}\right) \sim v_{2, q}^{n / 2}\left(p_{\mathrm{T}}\right)$, which leads naturally to the observed similar scaling relation among hadron anisotropic flows when the coalescence model is used to describe hadron production from the partonic matter.

\section{Two-particle interferometry}

The Hanbury-Brown Twiss (HBT) effect was first used to measure the size of an emission source like a star [214]. For heavy ion collisions, the HBT effect may provide information not only on the spatial extent of the emission source but also on its emission duration [215-218]. In particular, the long emission time as a result of the phase transition from the quark-gluon plasma to the hadronic matter in relativistic heavy ion collisions may lead to an emission source which has a $R_{\text {out }} / R_{\text {side }}$ ratio much larger than 1 [218-220], where the out-direction is along the total transverse momentum of two detected particles and the side-direction is perpendicular to both the out-direction and the beam direction (called the long-direction) [216,217]. Since the quark-gluon plasma is expected to be formed in heavy ion collisions at RHIC, it is thus surprising to find that the extracted ratio $R_{\text {out }} / R_{\text {side }}$ from a Gaussian fit to the measured correlation function of two identical pions in $\mathrm{Au}+\mathrm{Au}$ collisions is close to 1 [221-223]. This is in sharp contrast with calculations from hydrodynamic models [219,224], where $R_{\text {out }} / R_{\text {side }}$ is typically well above 1 .

Denoting the single-particle emission function by $S(x, \mathbf{p})$, the HBT correlation function for two identical bosons in the absence of final-state interactions is given by [215,225]:

$$
\begin{aligned}
& C_{2}(\mathbf{Q}, \mathbf{K}) \\
& \quad=1+\frac{\int d^{4} x_{1} d^{4} x_{2} S\left(x_{1}, \mathbf{K}\right) S\left(x_{2}, \mathbf{K}\right) \cos \left[Q \cdot\left(x_{1}-x_{2}\right)\right]}{\int d^{4} x_{1} S\left(x_{1}, \mathbf{p}_{1}\right) \int d^{4} x_{2} S\left(x_{2}, \mathbf{p}_{2}\right)},
\end{aligned}
$$

where $\mathbf{p}_{\mathbf{1}}$ and $\mathbf{p}_{\mathbf{2}}$ are momenta of the two hadrons, $\mathbf{K}=\left(\mathbf{p}_{\mathbf{1}}+\right.$ $\left.\mathbf{p}_{2}\right) / 2$, and $Q=\left(\mathbf{p}_{1}-\mathbf{p}_{2}, E_{1}-E_{2}\right)$. The three-dimensional correlation function in $\mathbf{Q}$ can be shown as one-dimensional functions of the projections of $\mathbf{Q}$ in the "out-side-long" coordinate system [216,217].

Expecting that the emission function is sufficiently smooth in momentum space, the size of the emission source can then be related to the emission function as

$$
\begin{aligned}
R_{i j}^{2}(K) & =-\left.\frac{1}{2} \frac{\partial^{2} C_{2}(\mathbf{Q}, \mathbf{K})}{\partial Q_{i} \partial Q_{j}}\right|_{\mathbf{Q}=0} \\
& =D_{x_{i}, x_{j}}(K)-D_{x_{i}, \beta_{j} t}(K)-D_{\beta_{i} t, x_{j}}(K)+D_{\beta_{i} t, \beta_{j} t}(K) .
\end{aligned}
$$

These source radii are thus expressed in terms of the spacetime variances $D_{x, y}=\langle x \cdot y\rangle-\langle x\rangle\langle y\rangle$, with $\langle x\rangle$ denoting the average value of $x$. In the above, $x_{i}(i=1-3)$ denote the projections of the particle position at freeze-out in the "outside-long" system, i.e., $x_{\text {out }}, x_{\text {side }}$, and $x_{\text {long }}$, respectively; and $\beta=\mathbf{K} / K_{0}$, with $K_{0}$ being the average energy of the two particles.

The experimentally measured two-particle correlation function $C_{2}(\mathbf{Q}, \mathbf{K})$ in central heavy ion collisions is usually fitted by a four-parameter Gaussian function after correcting for 
final-state Coulomb interactions, i.e.,

$$
C_{2}(\mathbf{Q}, \mathbf{K})=1+\lambda \exp \left(-\sum_{i=1}^{3} R_{i i}^{2}(K) Q_{i}^{2}\right) .
$$

If the emission source is Gaussian in space-time, the fitted radii $R_{i i}$ would be identical to the source radii determined from the emission function via Eq. (22). However, because of space-time correlations in the emission function, such as those induced by the collective flow, the fitted radii can be quite different from the source radii [226,227].

We used the AMPT model to study the interferometry of two identical pions or kaons in central $\mathrm{Au}+\mathrm{Au}$ collisions at RHIC $[48,50]$. The source of the emitted particles were obtained from their space-time coordinates $x$ and momenta $\mathbf{p}$ at kinetic freeze-out, i.e., at their last interactions. The correlation function $C_{2}(\mathbf{Q}, \mathbf{K})$ was then evaluated in the frame of longitudinally comoving system using the program Correlation After Burner [228]. In these calculations, the cutoff time $t_{\text {cut }}$ for hadron cascade was chosen as $200 \mathrm{fm} / c$ for HBT studies instead of the default $30 \mathrm{fm} / c$, because we are interested in the space-time and momentum distributions of hadrons at freeze-out even though their rapidity distributions and momentum spectra do not essentially change after $30 \mathrm{fm} / c$.

For central $(b=0 \mathrm{fm}) \mathrm{Au}+\mathrm{Au}$ collisions at $\sqrt{s_{N N}}=$ $200 \mathrm{GeV}$, results for charged pions from the AMPT model with string melting and parton cross section $\sigma_{p}=6 \mathrm{mb}$ are shown in Fig. 30 together with experimental data from the STAR [229] and PHOBOS [230] Collaborations. The STAR data at the three transverse momentum $k_{\mathrm{T}}$ bins of $(0.15,0.25),(0.25,0.35)$, and $(0.35,0.60) \mathrm{GeV} / c$ are for midrapidity and the $0-5 \%$ most central collisions, while the PHOBOS data are for the $0-15 \%$ most central collisions with $\left\langle y_{\pi \pi}\right\rangle=0.9$. We find that the source radii $R_{\text {out }}$ (upper-left

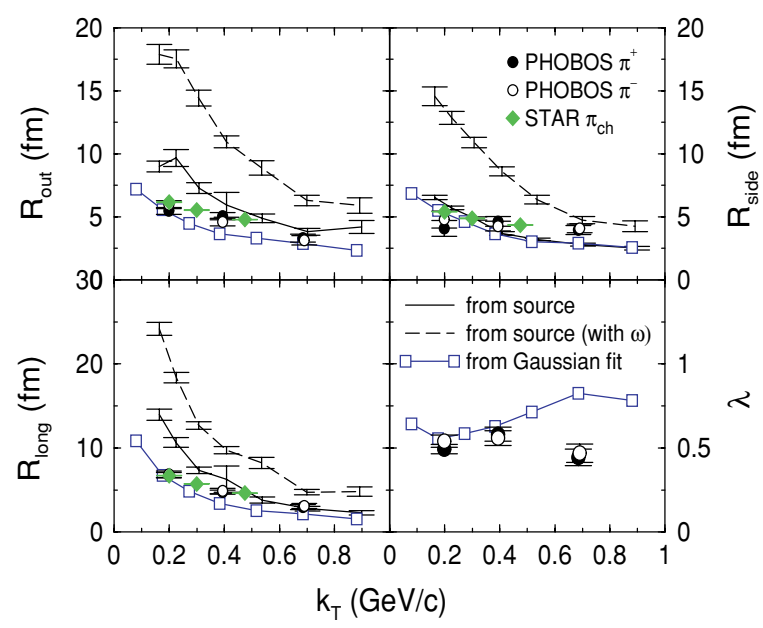

FIG. 30. (Color online) Pion source radii with (dashed curves) and without (solid curves) $\omega$ decays as well as the fitted radius and $\lambda$ parameters from the Gaussian fit to the correlation function (curves with squares) at midrapidity as functions of $k_{\mathrm{T}}$ from AMPT with string melting and $\sigma_{p}=6 \mathrm{mb}$ for central $\mathrm{Au}+\mathrm{Au}$ collisions at $\sqrt{s_{N N}}=$ $200 \mathrm{GeV}$. Circles are PHOBOS data; diamonds are STAR data. panel), $R_{\text {side }}$ (upper-right panel), and $R_{\text {long }}$ (lower-left panel) of pions including those from $\omega$ decays (dashed curves) are a factor of 2 to 3 larger than the radius parameters from a Gaussian fit to the three-dimensional correlation function obtained from the AMPT model (curves with squares) or to the experimentally measured one (open or filled circles). Excluding pions from $\omega$ decays reduces the source radii (solid curves), and this brings $R_{\text {side }}$ close to the fitted one while $R_{\text {out }}$ and $R_{\text {long }}$ can still be a factor of 2 larger than fitted ones. The emission source from the AMPT model thus deviates appreciably from a Gaussian one, as found previously in studies at SPS using the RQMD transport model [226,227]. In this case, it will be useful to compare the emission source from the AMPT model with that extracted from measured two-particle correlation functions using the imaging method [231,232].

Since Eq. (22) gives

$$
R_{\text {out }}^{2}=D_{x_{\text {out }}, x_{\text {out }}}-2 D_{x_{\text {out }}, \beta_{\perp} t}+D_{\beta_{\perp} t, \beta_{\perp} t},
$$

and $R_{\text {side }}{ }^{2}=D_{x_{\text {side }}, x_{\text {side }}}$, the ratio $R_{\text {out }} / R_{\text {side }}$ contains information about the duration of emission and has thus been studied extensively [218-221]. However, the $x_{\text {out }}-t$ distributions at freeze-out from the AMPT model show a strong positive $x_{\text {out }}-t$ correlation for both pions and kaons [48,50]. This leads to a positive $D_{x_{\text {out }}, \beta_{\perp} t}$ and thus a negative contribution to $R_{\text {out }}^{2}$ that can be as large as the positive duration-time term, making it difficult to extract information about the duration of emission from the ratio $R_{\text {out }} / R_{\text {side }}$ alone.

The dependence of fitted radii on $\sigma_{p}$, with $\sigma_{p}=0$ denoting the default AMPT model without string melting, is shown in Fig. 31 for midrapidity charged pions with $125<p_{\mathrm{T}}<$ $225 \mathrm{MeV} / c$. Also shown by circles, triangles, diamonds, and squares are the STAR data from central $\mathrm{Au}+\mathrm{Au}$ collisions for midrapidity pions with $125<p_{\mathrm{T}}<225 \mathrm{MeV} / c$ at $\sqrt{s_{N N}}=130 \mathrm{GeV}$ (open symbols) and for pions with $150<$ $p_{\mathrm{T}}<250 \mathrm{MeV} / c$ at $\sqrt{s_{N N}}=200 \mathrm{GeV}$ (filled symbols) for $R_{\text {out }}, R_{\text {side }}, R_{\text {long }}$, and $\lambda$ parameter, respectively. Both the

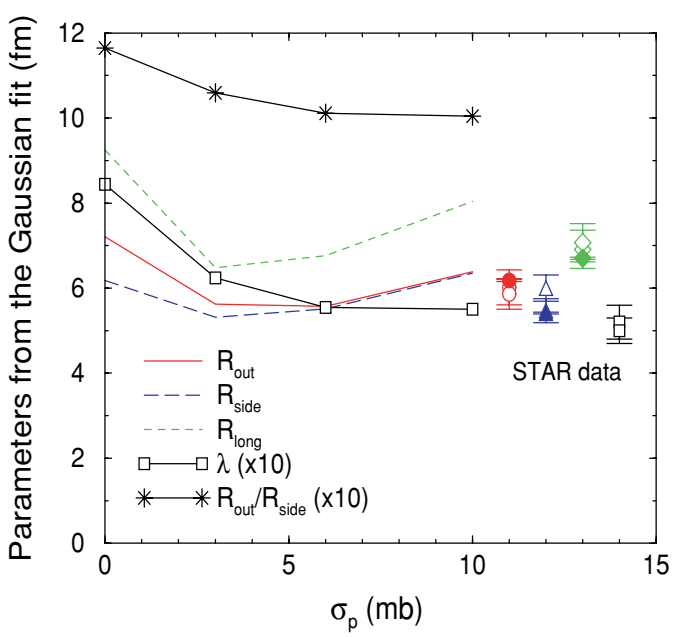

FIG. 31. (Color online) Radii, $\lambda$ parameters, and $R_{\text {out }} / R_{\text {side }}$ for midrapidity pions with $125<p_{\mathrm{T}}<225 \mathrm{MeV} / c$ from Gaussian fits of correlation function as functions of $\sigma_{p}$. STAR data at $\sqrt{s_{N N}}=130$ and $200 \mathrm{GeV}$ are shown by open and filled symbols, respectively. 


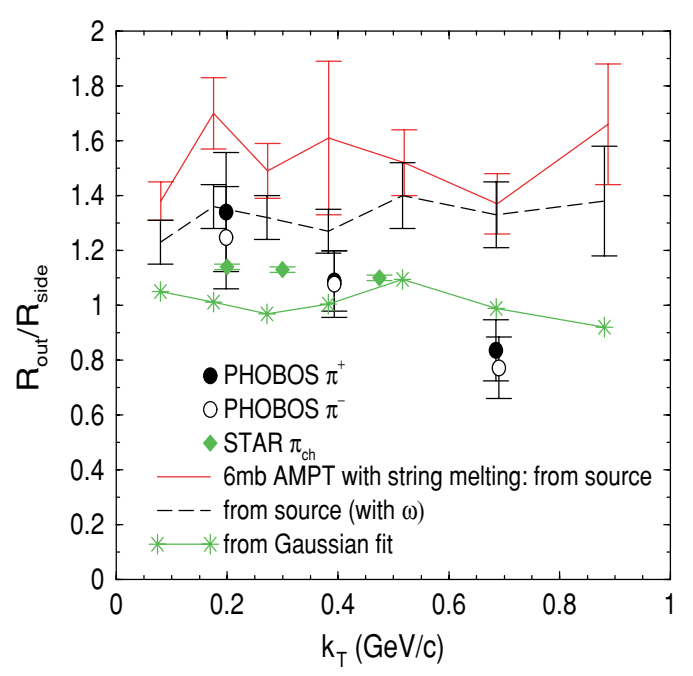

FIG. 32. (Color online) $R_{\text {out }} / R_{\text {side }}$ ratios for midrapidity pions at $\sqrt{s_{N N}}=200 \mathrm{GeV}$ as functions of $k_{\mathrm{T}}$. Solid and dashed curves are AMPT results from the emission function without and with pions from $\omega$ decays, respectively. The curve with stars is the AMPT result from the Gaussian fit to the two-pion correlation function.

radius and the $\lambda$ parameters from the AMPT model with string melting and $\sigma_{p}=3-10 \mathrm{mb}$ are close to the experimental values [221], while results from the default AMPT model overpredict the $\lambda$ parameter. For results with string melting, all three radius parameters are seen to increase with increasing parton cross section $\sigma_{p}$ presumably as a result of the larger source size at freeze-out, while the extracted $\lambda$ parameter decreases gradually with increasing $\sigma_{p}$.

Figure 32 shows the ratio $R_{\text {out }} / R_{\text {side }}$ for midrapidity charged pions from AMPT with string melting and $\sigma_{p}=6 \mathrm{mb}$ for central $(b=0 \mathrm{fm}) \mathrm{Au}+\mathrm{Au}$ collisions at $\sqrt{s_{N N}}=200 \mathrm{GeV}$. It is seen that the ratios obtained from the emission function have values between 1.0 and 1.7 , consistent with predictions from the hydrodynamic model with freeze-out treated via the hadronic transport model [219]. However, the ratio from the Gaussian fit to the three-dimensional correlation function (the curve with stars) is much closer to 1 , similar to that extracted from a Gaussian fit to the measured correlation function $[221,229,230]$.

The AMPT model with string melting is so far the only dynamical model that gives a $R_{\text {out }} / R_{\text {side }}$ ratio close to 1 and also gives roughly the correct magnitude for the fitted radii. Future studies using this transport model on the $x$ - $t$ correlation term [233] or on azimuthal HBT in noncentral heavy ion collisions will help us to further test the AMPT model and understand the freeze-out dynamics.

\section{RESULTS AT LHC}

The AMPT model can also be used to study heavy ion collisions at LHC. Figure 33 gives the charged-particle pseudorapidity distribution and the rapidity distributions of charged pions, kaons, protons, and antiprotons with and without nuclear shadowing in central $(b \leqslant 3 \mathrm{fm}) \mathrm{Pb}+\mathrm{Pb}$ collisions at $\sqrt{s_{N N}}=5.5 \mathrm{TeV}$ from the default AMPT model.
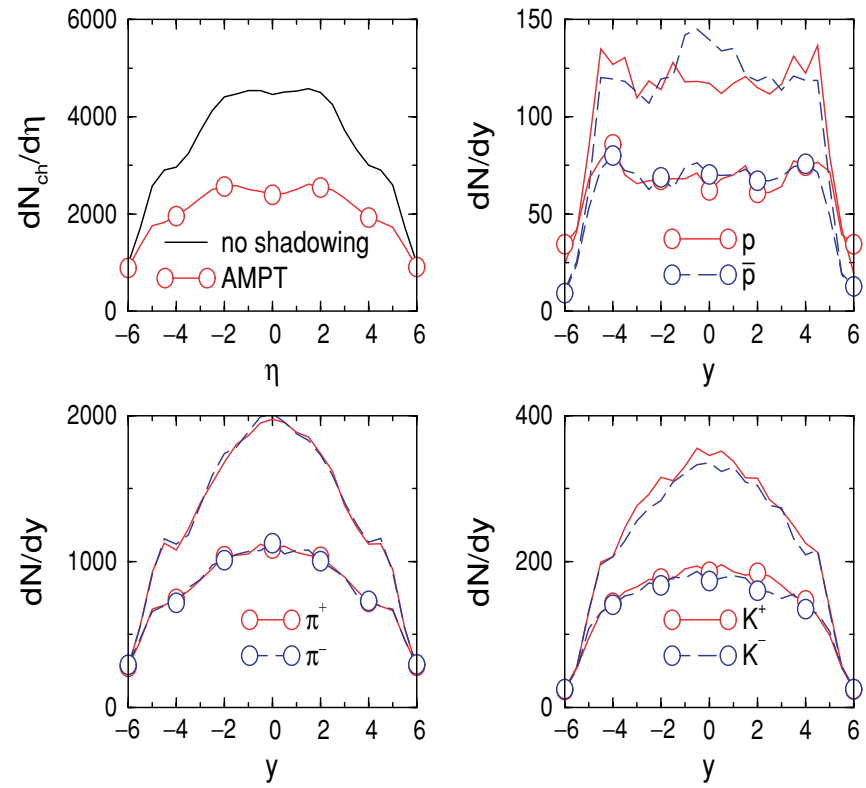

FIG. 33. (Color online) Rapidity distributions for central $(b \leqslant$ $3 \mathrm{fm}) \mathrm{Pb}+\mathrm{Pb}$ collisions at $\sqrt{s_{N N}}=5500 \mathrm{GeV}$ from the default AMPT model with (curves with circles) and without (curves with no symbols) nuclear shadowing.

The distributions are significantly wider and higher than corresponding distributions at RHIC. At midrapidity, the distributions without shadowing are higher than corresponding ones with shadowing by about $80 \%$. The highest value at midrapidity is about 4500, well within the LHC detector limit of 7000 particles per unit rapidity. The midrapidity density with nuclear shadowing is about 2500 , more than a factor of 3 higher than that at RHIC. It is higher than the logarithmic extrapolation from lower energy data but lower than the saturation model prediction of about 3500 [234]. The charged hadron pseudorapidity distribution shows a clear plateau structure which is very different from predictions from saturation models $[235,236]$. The proton and antiproton rapidity distributions are close to each other and almost flat. This is different from the proton and antiproton distributions at RHIC where protons clearly dominate at large rapidities. Note that the cutoff time $t_{\text {cut }}$ for the hadron cascade has been chosen as $200 \mathrm{fm} / c$ at LHC instead of the default $30 \mathrm{fm} / c$ because of the longer lifetime of the formed matter and the larger rapidity width in heavy ion collisions at LHC.

Transverse momentum spectra at LHC are shown in Fig. 34. It is seen that the inverse slope parameters, particularly for kaons and protons with transverse momenta below 0.5 and $1 \mathrm{GeV} / c$, respectively, are larger than at RHIC as a result of stronger transverse flows. Similar to that observed at RHIC, the proton spectrum is below that of pions at low transverse momenta, but they become comparable at about $2 \mathrm{GeV} / c$. As in heavy ion collisions at SPS (Fig. 19) and RHIC (Fig. 25), the strong transverse flow is due to final-state interactions; this is shown in Fig. 35, where the pion, kaon, and proton spectra at LHC obtained from the AMPT model with and without finalstate interactions are compared. 


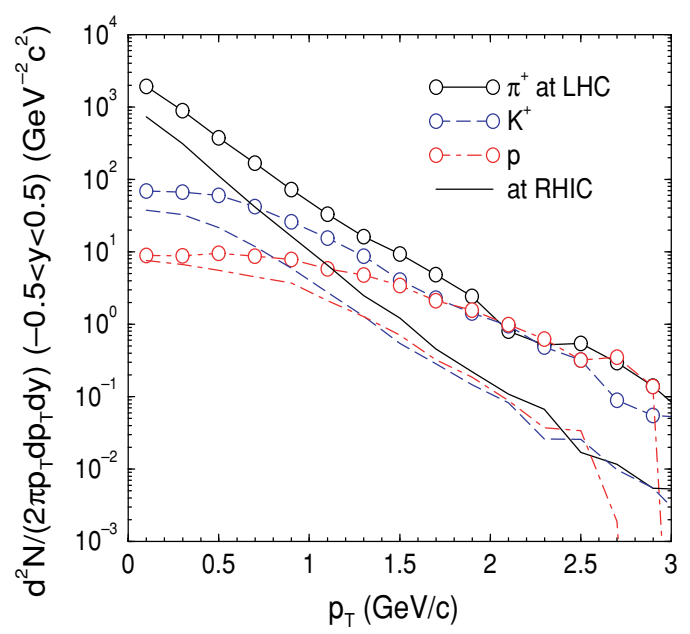

FIG. 34. (Color online) Transverse momentum spectra of pions, kaons, and protons from the default AMPT model for central $(b \leqslant$ $3 \mathrm{fm}) \mathrm{Pb}+\mathrm{Pb}$ collisions at $\sqrt{s_{N N}}=5500 \mathrm{GeV}$ (curves with circles) and for central $(b \leqslant 3 \mathrm{fm}) \mathrm{Au}+\mathrm{Au}$ collisions at $\sqrt{s_{N N}}=200 \mathrm{GeV}$ (curves with no symbols).

Because of the uncertainty in the parton distribution functions at small $x$ in heavy nuclei, the above results on LHC have large uncertainties. Currently, the initial condition of the AMPT model is obtained from the HIJING model with minijets and strings. The parton distribution functions used in the HIJING model are the Duke-Owens set 1 [62], which are quite old. For example, Fig. 36 shows the gluon and $u$-quark distribution functions at $Q^{2}=9 \mathrm{GeV}^{2}$ from the Duke-Owens set 1 (circles), MRSA [237] (squares), and the recent four-flavor CTEQ6M [238] (diamonds) parametrizations. It is seen that the Duke-Owens set 1 parametrization has far fewer partons at small $x$, e.g., when $x<0.01$, than the other two more recent parametrizations. At the top RHIC energy, a pair of $2 \mathrm{GeV}$ back-to-back minijets at midrapidity corresponds to $x \sim 0.02$ for the initial partons, and at the LHC energy it corresponds to

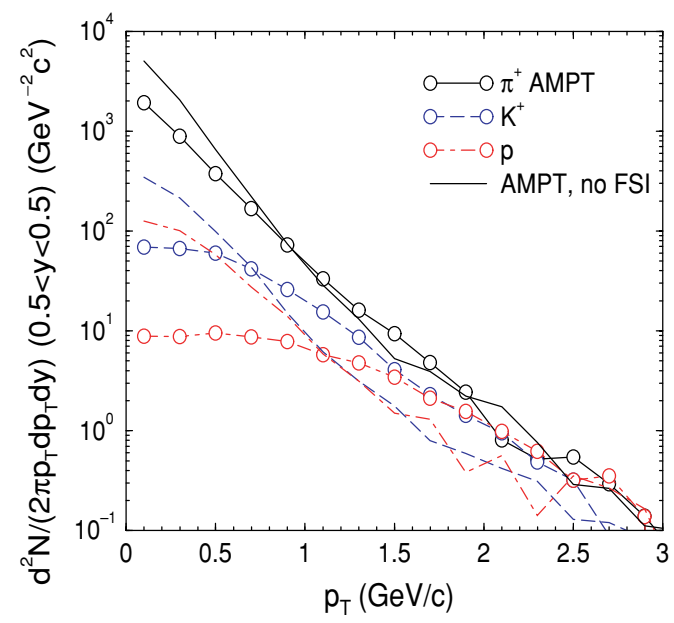

FIG. 35. (Color online) Transverse momentum spectra of pions, kaons, and protons for central $(b \leqslant 3 \mathrm{fm}) \mathrm{Pb}+\mathrm{Pb}$ collisions at $\sqrt{s_{N N}}=$ $5500 \mathrm{GeV}$ from the default AMPT model with (curves with circles) and without (curves with no symbols) final-state interactions.

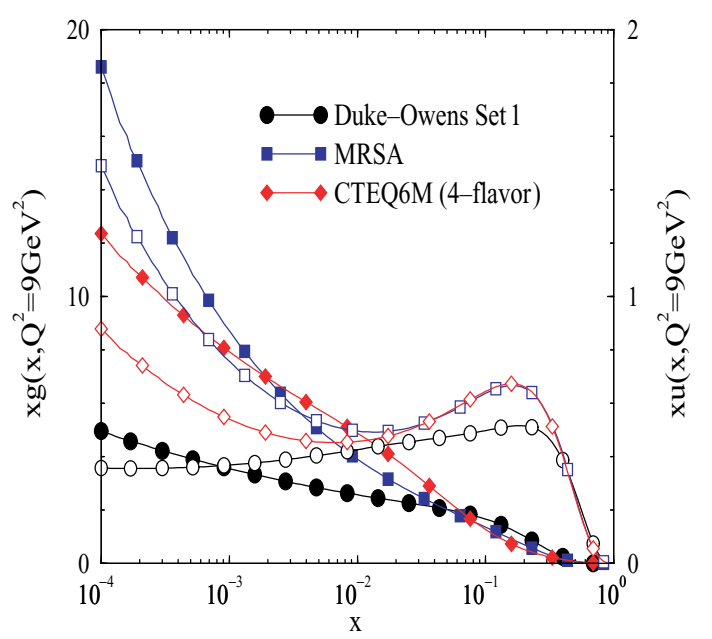

FIG. 36. (Color online) Gluon (curves with filled symbols) and $u$-quark (curves with open symbols) distribution functions (multiplied by $x$ ) at $Q^{2}=9 \mathrm{GeV}^{2}$ from three different parametrizations.

$x \sim 0.00073$. Thus small- $x$ partons play much more important roles at LHC than at RHIC, and the AMPT model will have a much larger uncertainty at LHC because of the underestimate of small- $x$ partons from the Duke-Owens set 1 . An update of the parton distribution functions for the HIJING model has been done, [63] in which the Gluck-Reya-Vogt parton distribution functions [239] have been implemented together with a new nuclear shadowing parametrization. To make predictions with better accuracy for LHC, we will need to update the parton distribution functions in a future version of the AMPT model.

\section{DISCUSSIONS}

Two versions of the AMPT model have been used in the present study. The default AMPT model (version 1.11), which includes only minijet partons in the parton cascade and uses the Lund string model for hadronization, is found to give a reasonable description of hadron rapidity distributions and transverse momentum spectra observed in heavy ion collisions at both SPS and RHIC. It underpredicts, however, the magnitude of the elliptic flow and fails to reproduce the $\lambda$ parameter of the two-pion correlation function measured at RHIC. The latter can be described, on the other hand, by the AMPT model with string melting (version 2.11) when the parton scattering cross section is about $6 \mathrm{mb}$, as shown in Secs. V H and VI. This extended model underpredicts, however, the inverse slopes of hadron transverse momentum spectra and fails to describe the baryon rapidity distributions, as shown in Figs. 37 and 38, respectively. Since the AMPT model has not been able to describe all experimental observables at RHIC within a single version, further improvements are needed.

The initial condition of the AMPT model is obtained from the HIJING model with minijets and strings. Although the string melting mechanism $[45,48,50]$ is introduced to convert the energy in initial excited strings into partons in order to better model the partonic initial condition at high energy densities, it is modeled by using the Lund string fragmentation 


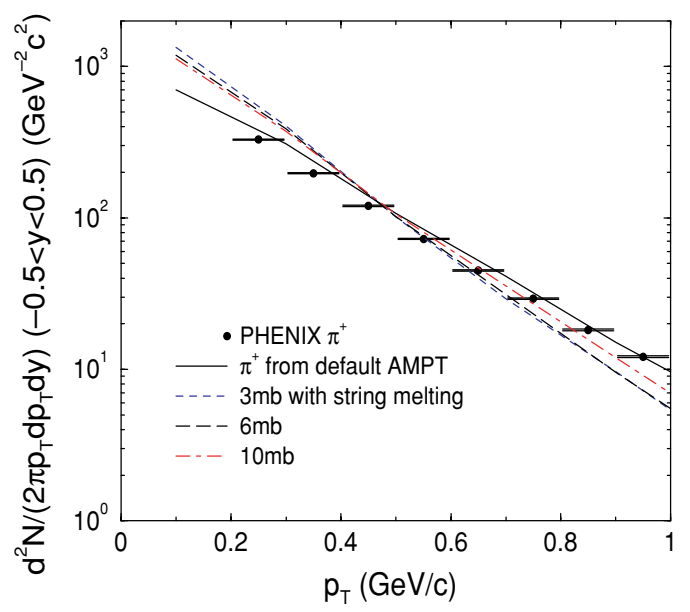

FIG. 37. (Color online) Comparison of transverse momentum spectra of midrapidity pions from the AMPT model and the PHENIX data [123] for the $5 \%$ most central $\mathrm{Au}+\mathrm{Au}$ collisions at $\sqrt{s_{N N}}=$ $200 \mathrm{GeV}$.

to hadrons as an intermediate process and then converting these hadrons into their valence quarks. This is equivalent to staying at the parton level until strings in the Lund model have generated all the quark-antiquark pairs before forming hadrons. The current string melting can be viewed as a minimal implementation because in the limit of no partonic interactions (and before hadron cascade starts), it reduces to HIJING results for all hadrons other than the flavor-diagonal mesons within the $\operatorname{SU}(2)$ flavor space, $\pi^{0}, \eta, \rho^{0}$, and $\omega$. As a result, the initial partonic matter in this scenario does not contain gluons.
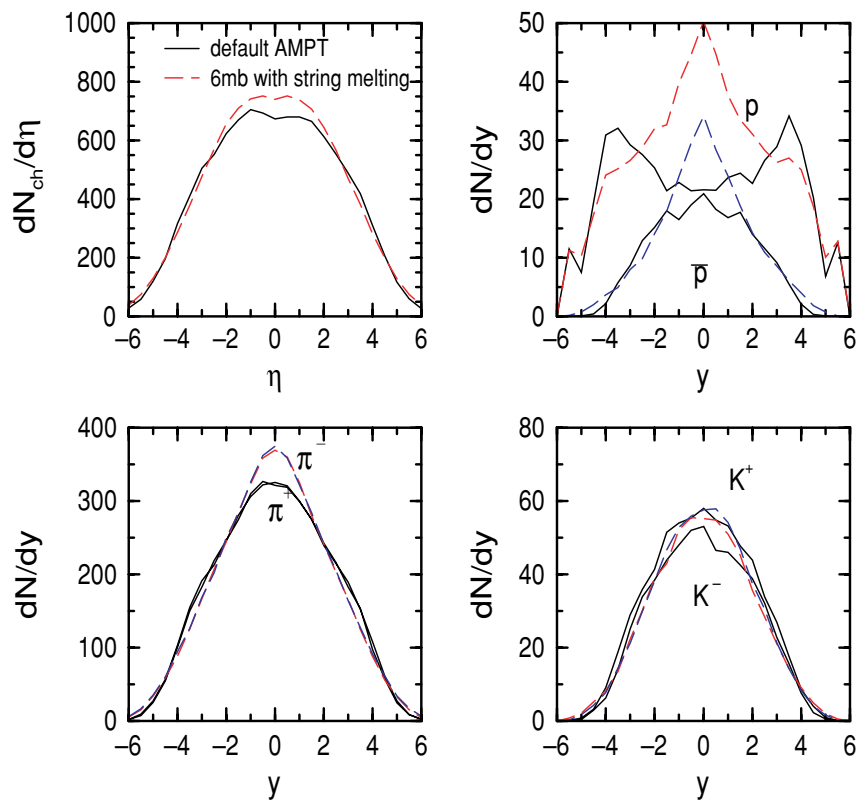

FIG. 38. (Color online) Rapidity distributions of total charged particles (upper left panel), protons and antiprotons (upper right panel), charged pions (lower left panel), and charged kaons (lower right panel) in the $5 \%$ most central $\mathrm{Au}+\mathrm{Au}$ collisions at $\sqrt{s_{N N}}=$ $200 \mathrm{GeV}$ from the default AMPT model (solid curves) and the AMPT model with string melting and $\sigma_{p}=6 \mathrm{mb}$ (dashed curves).
Although this is unphysical as one expects gluons to dominate the initial stage of ultrarelativistic heavy ion collisions, our study of the elliptic flow [45] and the pion interferometry [48] depends more on the effect of partonic scatterings instead of the composition of the partonic matter. However, for dilepton production from the partonic matter, the flavor composition of the partonic matter is important [240], and at present they cannot be addressed within the AMPT model. To extend the string melting scenario to include gluons in the AMPT model, we need to study the problem of both quark-antiquark and gluon production from a strong color field. Also, alternative descriptions of initial conditions, such as that from the parton saturation model [29-32,34-37], may also be used as input to the AMPT model.

The parton cascade model ZPC in the AMPT model only includes leading-order two-body partonic interactions (2-to2 ), while higher-order processes ( $m$-to- $n$ in general), which might become dominant at high densities during the early stage of relativistic heavy ion collisions [241], have not been included. Unfortunately, it is difficult to implement many-body interactions in a transport model, even for the next-leading-order processes ( $g g \leftrightarrow g g g$ ) in a parton cascade [242]. Also, we currently treat the screening mass $\mu$ as a parameter for changing the parton scattering cross sections. In principle, it should be evaluated dynamically from the effective temperature of the evolving parton system, thus leading to medium-dependent parton scattering cross sections.

Furthermore, treating interactions at a distance as in the cascade model used for solving the Boltzmann equations leads to possible violation of causality when the mean free path of partons is shorter than their interaction length, i.e.,

$$
\lambda=\frac{1}{\rho \sigma}<\sqrt{\frac{\sigma}{\pi}} \text { or } \frac{\rho^{2} \sigma^{3}}{\pi}>1 .
$$

If we take the parton density as $\rho=10 / \mathrm{fm}^{3}$ at RHIC and take the parton scattering cross section as $\sigma=3 \mathrm{mb}$, then we have $\rho^{2} \sigma^{3} / \pi=0.86$ and thus expect that the usual method used in the cascade model for solving the Boltzmann equation may not be very accurate. However, the Boltzmann equation with only two-body scatterings is invariant under the transformation

$$
f \rightarrow f l \text { and } \sigma \rightarrow \frac{\sigma}{l},
$$

but the value of $\rho^{2} \sigma^{3} / \pi$ is reduced by a factor of $l$. With sufficiently large $l$, the so-called parton subdivision can then overcome the causality problem in the cascade method [18,23,201,243-245]. Although parton subdivision has been implemented in ZPC, it remains to be implemented in the AMPT model mainly due to the complication in the hadronization process which converts partons into hadrons following the parton cascade. How the causality violation in the AMPT model affects the final observables in heavy ion collisions [201] is yet to be studied.

In the hadronization process in the default AMPT model, a minijet recombines with its parent string even though the minijet has gone through partonic interactions and thus has changed its color charge. The HIJING model also takes a similar approach in the jet quenching process because a gluon is still being associated with the same parent string after 
it goes through inelastic collisions. Our current prescription guarantees that fragmentation can successfully proceed for all strings after parton interactions; it also enables the default AMPT model to reduce to HIJING results in the limit of no partonic (and hadronic) interactions. Although the freezeout positions and times of minijet partons are averaged in a string before its fragmentation, the current treatment of color configurations certainly needs to be improved; that is, new color-singlet strings over the whole volume need to be reconstructed after partonic interactions.

To describe charged-particle multiplicities in heavy ion collisions at SPS, we changed the values of the $a$ and $b$ parameters in the Lund symmetric fragmentation function given by Eq. (9) based on the possibility that these parameters could be modified in the dense matter formed in heavy ion collisions. However, the AMPT model needs the default $a$ and $b$ values, i.e., the values in the HIJING model, in order to reproduce the charged-particle multiplicities in $p p$ and $p \bar{p}$ collisions. The $a$ and $b$ values in the AMPT model is thus expected to depend on the atomic weight of colliding nuclei and the centrality of their collisions. Although the AMPT results on $d+$ Au collisions using default $a$ and $b$ values [49] agree reasonably well with the RHIC $d+\mathrm{Au}$ data [246-248], the detailed dependence of $a$ and $b$ parameters on the system size has not been studied.

At present, phase transition in the AMPT model with string melting is modeled by a simple quark coalescence using the current quark masses. The failure of the proton rapidity distribution in this model, shown in Fig. 38, is related to this assumption as the proton mass is not generated dynamically but is given to the coalescing system of three light quarks. In the case that the invariant mass of the three quarks is small, the resulting proton with its physical mass tends to overpopulate at midrapidity. This problem may be avoided if protons are formed from coalescence of quarks with constituent quark masses. However, the current string melting mechanism would fail to convert strings into partons since the pion, as a Goldstone boson, cannot be decomposed into a quark and antiquark with constituent quark masses. A more consistent method is perhaps to use density- or temperature-dependent quark masses, so that they correspond to current masses at high temperature and constituent masses at low temperature near the phase transition, where a scalar field is responsible for the changing masses. This method also has the advantage that it can qualitatively describe the equation of state of the QGP [182]. Also, the current coalescence model could have problems with entropy, because quark coalescence reduces the number of particles by a factor of 2 to 3 , although entropy also depends on the degeneracy and mass of produced hadrons. We have studied the entropy problem in a schematic model using a thermalized QGP with the same parameters as given in Ref. [209]. Because of productions of massive resonances such as $\rho, \omega$, and $\Delta$, the total entropy is reduced by about $15 \%$ when partons are converted to hadrons via coalescence, and this is mainly due to a similar violation in energy. To eliminate entropy violation thus requires a proper treatment of energy conservation. This requires the inclusion of field energy in both QGP and hadronic matter, which is also necessary to properly describe the equation of state. Furthermore, although the spatial correlation in the quark coalescence based on the closest neighbors leads to momentum correlation among coalescing partons in the presence of collective flow, explicit momentum correlation is not considered in our quark coalescence model, and random momentum distributions of coalescing quarks tend to give the artificial peak at midrapidity shown in Fig. 38. The quark coalescence model can be improved by following the method used recently in studying hadron production from the quark-gluon plasma, which has been shown to describe satisfactorily the observed quark number scaling of hadron elliptic flows and large baryon-to-pion ratio at intermediate transverse momenta [137-145]. Also, statistical models that generate hadronic states according to their statistical weights provide an alternative method for describing the parton-tohadron phase transition.

Many of the hadronic cross sections used in the hadronic stage of the AMPT model have not been studied theoretically in detail not well constrained by experimental data, and they may be important for some observables. For example, from the study at SPS energies with the default AMPT model, we found that the explicit inclusion of $K^{*}$ mesons and baryonantibaryon production from two-meson states are important for strangeness and antiproton production, respectively. In the AMPT model with string melting, the phase transition happens later than in the default AMPT model because of the larger energy and lifetime of the partonic system; therefore, hadronic effects are less important than in the default AMPT model. Even in this case, it is essential to know the cross sections of $\eta$ meson interactions with other hadrons as they may affect the final $\eta$ yield and consequently the height (or the $\lambda$ parameter) of the two-pion correlation function. Effects due to the uncertainties of these hadronic input parameters on final observables at RHIC will be studied in the future.

\section{SUMMARY}

To study high-energy heavy ion collisions at SPS and RHIC and even higher energies at the LHC, a multiphase transport (AMPT) model has been developed. It consists mainly of four components: the HIJING model to convert the initial incident energy to the production of hard minijet partons and soft strings, with excited strings further converting to partons in the AMPT model with string melting; ZPC to model the interactions among partons; the Lund string fragmentation as implemented in JETSET/PYTHIA to convert the excited strings to hadrons in the default model or a simple quark coalescence model to convert partons into hadrons in the case of string melting; and the extended relativistic transport (ART) model for describing interactions among hadrons. In this paper, we have described in detail the physics input in each component and how the different components are combined to give a comprehensive description of the dynamics of relativistic heavy ion collisions. We have used this model to study various observables in heavy ion collisions and to address the relative importance of partonic and hadronic effects on these observables. In particular, the AMPT model has been used to study the rapidity distributions of particles such as pions, kaons, protons, and antiprotons, their transverse momentum spectra, the elliptic flow, and the 
interferometry of two identical mesons. We find that the default AMPT model (version 1.11) gives a reasonable description of rapidity distributions and transverse momentum spectra, while the AMPT model with string melting (version 2.11) describes both the magnitude of the elliptic flow at midrapidity and the pion correlation function with a parton cross section of about $6 \mathrm{mb}$.

The high-energy heavy ion collision is a complex process involving the initial conditions, the interactions of initially produced partons and of later hadronic matter, as well as the transition between these two phases of matter. The AMPT model is an attempt to incorporate these different physics as much as we can at present. Since there are many uncertainties in the AMPT model, the model is currently more a simulation tool than a finalized code. Nevertheless, this model provides the possibility to study the dependence of various observables on these physical effects. For example, we found that the elliptic flows are sensitive to early parton dynamics, and the HBT interferometry is instead affected by the complicated late hadron freeze-out dynamics. The AMPT model can be extended, e.g., to include hydrodynamic evolution at the early stage when local thermalization is likely, in order to conveniently study the equation of state of the partonic matter. Experiments at RHIC such as $d+\mathrm{Au}$ can also help to reduce the uncertainties in the physical input such as the parton distribution functions in heavy nuclei. With continuing efforts in both theoretical and experimental heavy ion physics, we hope that this multiphase transport model will eventually incorporate the essential elements of the underlying theory of QCD to provide a reliable description of different observables in heavy ion collisions within one coherent picture, and help us to learn from relativistic heavy ion collisions the properties of the quark-gluon plasma formed during the early stage of the collisions.

\section{ACKNOWLEDGMENTS}

We thank L. W. Chen, V. Greco, M. Gyulassy, U. Heinz, H. Huang, D. Molnar, M. Murray, and N. Xu for useful discussions. Z.W.L. and B.Z. thank the Department of Energy's Institute for Nuclear Theory at the University of Washington for hospitality during the INT-03-1 program "The First Three Years of Heavy Ion Physics at RHIC," where part of the work was done. We also thank the Parallel Distributed System Facility at the National Energy Research Scientific Computer Center for providing computer resources. This work was supported by the U.S. National Science Foundation under Grant Nos. PHY-0098805 (Z.W.L., S.P., and C.M.K.), PHY-0457265 (C.M.K.), PHY-0354572 (B.A.L.), and PHY-0140046 (B.Z.), the U.S. Department of Energy under Grant No. DE-FG0201ER41190 (Z.W.L.), and the Welch Foundation under Grant No. A-1358 (Z.W.L., S.P., and C.M.K.).

\section{APPENDIX: AMPT USERS' GUIDE}

The default AMPT model (version 1.11) and the AMPT model with string melting (version 2.11) both use an initialization file 'input.ampt'. The analysis directory 'ana/' contains the resulting data files. The final particle record file is 'ana/ampt.dat'. The version number of AMPT is written to both 'ana/version' and 'nohup.out' files. The AMPT source code has been tested for both Fortran 77 and the Portland Group Fortran 77 compilers on the UNIX, Linux, and OSF1 operating systems.

To run the AMPT program, one needs to

(i) Set the initial parameters in 'input.ampt'. If one prefers to use runtime random number seed, set 'ihjsed $=11$ ', In this way, every run is different even with the same 'input.ampt' file.

(ii) Type 'sh exec \&' to compile and run the executable 'ampt' with some general information written in 'nohup.out'.

Key initial parameters in 'input.ampt' are

EFRM: $\sqrt{s_{N N}}$ in $\mathrm{GeV}$, e.g., 200 for the maximum RHIC energy.

NEVNT: the total number of events.

BMIN, BMAX: the minimum and maximum impact parameters (in fm) for all events with BMAX having an upper limit of HIPR1(34)+HIPR1(35) (=19.87 fm for $d+$ Au collisions and $25.60 \mathrm{fm}$ for $\mathrm{Au}+\mathrm{Au}$ collisions).

ISOFT: choice of parton-hadron conversion scenario.

$=1$ : default AMPT model (version 1.x);

=4: the AMPT model with string melting (version 2.y). Note that values of 2, 3, and 5 have never been used for publications. They are tests of other string melting scenarios:

$=2$ : a string is decomposed into $q+q q+$ minijet partons instead of using the Lund fragmentation;

$=3$ : a baryon is decomposed into $q+q q$ instead of three quarks;

$=5$ : same as 4 , but partons freeze-out according to local energy density.

NTMAX: the number of time-steps for hadron cascade, default(D) $=150$. Note that NTMAX $=3$ effectively turns off hadron cascade, and a larger than the default value is usually necessary for observables at large rapidity or large pseudorapidity. We use NTMAX $=1000$ for HBT studies in central $\mathrm{Au}+\mathrm{Au}$ collisions because of the need for the space-time information of last interactions and for LHC calculations due to the longer lifetime of the formed matter.

DT: value of the time-step (in $\mathrm{fm} / c$ ) for hadron cascade, $\mathrm{D}=$ 0.2 . Note that $t_{\text {cut }}=\mathrm{NTMAX} \times \mathrm{DT}$ is the termination time of hadron cascade.

PARJ(41): parameter $a$ in the Lund symmetric fragmentation function.

PARJ(42): parameter $b$ in the Lund symmetric fragmentation function (in $\mathrm{GeV}^{-2}$ ). Note that we use default value in HIJING $(a=0.5$ and $b=0.9)$ for $d+\mathrm{Au}$ collisions, and $a=2.2$ and $b=0.5$ for collisions of heavy nuclei.

flag for popcorn mechanism: $\mathrm{D}=1$ (Yes) turns on the popcorn mechanism. In general, it increases baryon stopping.

PARJ(5): controls $\mathrm{BM} \overline{\mathrm{B}}$ vs $\mathrm{B} \overline{\mathrm{B}}$ in the popcorn mechanism, $\mathrm{D}=1.0$. 
shadowing flag: $\mathrm{D}=1$ (Yes) turns on nuclear shadowing. quenching flag: $\mathrm{D}=0$ (No) turns off jet quenching since the ZPC simulates final-state effects.

p0 cutoff: $\mathrm{D}=2.0$ (in $\mathrm{GeV} / c$ ) for $p_{0}$ in HIJING for minijet production.

parton screening mass: controls the parton cross section, $\mathrm{D}=$ $3.2264\left(\right.$ in $\left.^{-1}\right)$. Its square is inversely proportional to the parton cross section. Use $\mathrm{D}=3.2264$ for $3 \mathrm{mb}$, and 2.2814 for $6 \mathrm{mb}$.

ihjsed: choice of the random number seed, $\mathrm{D}=0$.

$=0$ : take the 'Ran Seed for HIJING' in 'input.ampt' and disregard the random value generated in the file 'exec'. =11: take the HIJING random seed at runtime from the file 'exec,' with the seed written in 'nohup.out' and 'ana/version.'

Ran Seed for HIJING: random number seed for HIJING when ihjsed $=0$.

Kshort decay flag: depends on the experimental correction procedure, $\mathrm{D}=0$ turns off Kshort decays after the hadron cascade. Note that decays of the following resonances and their antiparticles are always included: $\rho, \omega, \eta, \mathrm{K}^{*}, \phi, \Delta$, $\mathrm{N}^{*}(1440), \mathrm{N}^{*}(1535), \Sigma^{0}$ (in order to include its feed down to $\Lambda$ ).

optional OSCAR output: if set to 1, outputs in OSCAR1997A format [60] are written in 'ana/parton.oscar' and 'ana/hadron.oscar.'

dpcoal: parton coalescence distance in momentum space (in $\mathrm{GeV} / c)$.

drcoal: parton coalescence distance in coordinate space (in $\mathrm{fm}$ ). dpcoal, drcoal both have $\mathrm{D}=10^{6}$ for nearest-neighbor coalescence in the AMPT model with string melting.
Key output files are

ana/ampt.dat: It contains particle records at hadron kinetic freeze-out, i.e., at the last interaction. For each event, the first line gives event number, test number $(=1)$, number of particles in the event, impact parameter, total number of participant nucleons in projectile, total number of participant nucleons in target, number of participant nucleons in projectile due to elastic collisions, number of participant nucleons in projectile due to inelastic collisions, and corresponding numbers in target. Note that participant nucleon numbers include nucleons participating in both elastic and inelastic collisions. Each of the following lines gives PYTHIA particle ID number, three-momentum $\left(p_{x}, p_{y}, p_{z}\right)$, mass, and space-time coordinates $(x, y, z, t)$ of one final particle at freeze-out. Note that momenta are in units of $\mathrm{GeV} / c$, mass in $\mathrm{GeV} / c^{2}$, space in $\mathrm{fm}$, and time in $\mathrm{fm} / c$. If a particle comes from the decay of a resonance that still exists at the termination time of hadron cascade, then its space-time corresponds to the decay point of the parent resonance. Also note that the $x$ axis in the AMPT program is defined as the direction along the impact parameter, and the $z$ axis is defined as the beam direction.

ana/ZPC.dat: similar to 'ana/ampt.dat' but for partons. The first line of each event gives event number, number of partons in the event, impact parameter, number of participant nucleons in projectile due to elastic collisions, number of participant nucleons in projectile due to inelastic collisions, and corresponding numbers in target. Each of the following lines gives: PYTHIA particle ID number, threemomentum $\left(p_{x}, p_{y}, p_{z}\right)$, mass, and space-time coordinates $(x, y, z, t)$ of one final parton at freeze-out.
[1] P. Braun-Munzinger, J. Stachel, J. P. Wessels, and N. Xu, Phys. Lett. B344, 43 (1995).

[2] J. Cleymans and K. Redlich, Phys. Rev. Lett. 81, 5284 (1998).

[3] P. Braun-Munzinger, I. Heppe, and J. Stachel, Phys. Lett. B465, 15 (1999).

[4] F. Becattini, J. Cleymans, A. Keranen, E. Suhonen, and K. Redlich, Phys. Rev. C 64, 024901 (2001).

[5] C. M. Hung and E. V. Shuryak, Phys. Rev. Lett. 75, 4003 (1995).

[6] D. H. Rischke, S. Bernard, and J. A. Maruhn, Nucl. Phys. A595, 346 (1995).

[7] D. H. Rischke, Y. Pursun, and J. A. Maruhn, Nucl. Phys. A595, 383 (1995); A596, 717 (E)(1996).

[8] P. F. Kolb, P. Huovinen, U. W. Heinz, and H. Heiselberg, Phys. Lett. B500, 232 (2001).

[9] P. Huovinen, P. F. Kolb, U. W. Heinz, P. V. Ruuskanen, and S. A. Voloshin, Phys. Lett. B503, 58 (2001).

[10] P. F. Kolb, U. W. Heinz, P. Huovinen, K. J. Eskola, and K. Tuominen, Nucl. Phys. A696, 197 (2001).

[11] D. Teaney, J. Lauret, and E. V. Shuryak, nucl-th/0110037.

[12] H. Sorge, H. Stocker, and W. Greiner, Nucl. Phys. A498, 567C (1989).

[13] Y. Pang, T. J. Schlagel, and S. H. Kahana, Phys. Rev. Lett. 68, 2743 (1992).

[14] B. A. Li and C. M. Ko, Phys. Rev. C 52, 2037 (1995).
[15] B. H. Sa and A. Tai, Comput. Phys. Commun. 90, 121 (1995).

[16] H. Sorge, Phys. Rev. C 52, 3291 (1995).

[17] S. Jeon and J. Kapusta, Phys. Rev. C 56, 468 (1997).

[18] B. Zhang, Comput. Phys. Commun. 109, 193 (1998).

[19] S. A. Bass et al., Prog. Part. Nucl. Phys. 41, 225 (1998).

[20] T. J. Humanic, Phys. Rev. C 57, 866 (1998).

[21] A. Tai and B. H. Sa, Comput. Phys. Commun. 116, 353 (1999).

[22] D. E. Kahana and S. H. Kahana, Phys. Rev. C 58, 3574 (1998).

[23] D. Molnar and M. Gyulassy, Phys. Rev. C 62, 054907 (2000).

[24] D. E. Kahana and S. H. Kahana, Phys. Rev. C 63, 031901(R) (2001).

[25] B. Li, A. T. Sustich, B. Zhang, and C. M. Ko, Int. J. Mod. Phys. E 10, 267 (2001).

[26] B. H. Sa, X. Cai, Z. D. Su, A. Tai, and D. M. Zhou, Phys. Rev. C 66, 044902 (2002).

[27] Y. L. Dokshitzer, V. A. Khoze, A. H. Mueller, and S. I. Troian, in Basics of Perturbative QCD (Editions Frontières, Gif-sur-Yvette, France, 1991), p. 274; R. Brock et al. (CTEQ Collaboration), Rev. Mod. Phys. 67, 157 (1995).

[28] X. N. Wang, Phys. Rep. 280, 287 (1997).

[29] L. McLerran and R. Venugopalan, Phys. Rev. D 49, 2233 (1994).

[30] L. McLerran and R. Venugopalan, Phys. Rev. D 49, 3352 (1994). 
[31] Y. V. Kovchegov and D. H. Rischke, Phys. Rev. C 56, 1084 (1997).

[32] D. Kharzeev and E. Levin, Phys. Lett. B523, 79 (2001).

[33] D. Kharzeev, E. Levin, and M. Nardi, Nucl. Phys. A730, 448 (2004); A743, 329(E) (2004).

[34] D. Kharzeev and M. Nardi, Phys. Lett. B507, 121 (2001).

[35] K. J. Eskola, K. Kajantie, P. V. Ruuskanen, and K. Tuominen, Nucl. Phys. B570, 379 (2000).

[36] K. J. Eskola, K. Kajantie, and K. Tuominen, Phys. Lett. B497, 39 (2001).

[37] K. J. Eskola, K. Kajantie, P. V. Ruuskanen, and K. Tuominen, Phys. Lett. B543, 208 (2002).

[38] R. Baier, A. H. Mueller, D. Schiff, and D. T. Son, Phys. Lett. B502, 51 (2001).

[39] B. Zhang, C. M. Ko, B. A. Li, and Z. W. Lin, in Atlanta 1999, Heavy Ion Physics from Bevalac to RHIC, edited by R. Seto (World Scientific, Singapore, 1999), p. 146.

[40] B. Zhang, C. M. Ko, B. A. Li, and Z. W. Lin, Phys. Rev. C 61, 067901 (2000).

[41] B. Zhang, C. M. Ko, B. A. Li, Z. W. Lin, and B. H. Sa, Phys. Rev. C 62, 054905 (2000).

[42] Z. W. Lin, S. Pal, C. M. Ko, B. A. Li, and B. Zhang, Phys. Rev. C 64, 011902(R) (2001).

[43] Z. W. Lin, S. Pal, C. M. Ko, B. A. Li, and B. Zhang, Nucl. Phys. A698, 375 (2002); also see http://nt4.phys.columbia. edu/people/zlin/PUBLICATIONS/lin-qm01.pdf.

[44] S. Pal, C. M. Ko, and Z. W. Lin, Nucl. Phys. A730, 143 (2004).

[45] Z. W. Lin and C. M. Ko, Phys. Rev. C 65, 034904 (2002).

[46] B. Zhang, C. M. Ko, B. A. Li, Z. W. Lin, and S. Pal, Phys. Rev. C 65, 054909 (2002).

[47] S. Pal, C. M. Ko, and Z. W. Lin, Nucl. Phys. A707, 525 (2002).

[48] Z. W. Lin, C. M. Ko, and S. Pal, Phys. Rev. Lett. 89, 152301 (2002).

[49] Z. W. Lin and C. M. Ko, Phys. Rev. C 68, 054904 (2003).

[50] Z. W. Lin and C. M. Ko, J. Phys. G 30, S263 (2004).

[51] X. N. Wang, Phys. Rev. D 43, 104 (1991).

[52] X. N. Wang and M. Gyulassy, Phys. Rev. D 44, 3501 (1991).

[53] X. N. Wang and M. Gyulassy, Phys. Rev. D 45, 844 (1992).

[54] M. Gyulassy and X. N. Wang, Comput. Phys. Commun. 83, 307 (1994).

[55] Version 1.383 of the HIJING program is available at http://www-nsdth.lbl.gov/ xnwang/hijing/dload.html.

[56] G. C. Rossi and G. Veneziano, Nucl. Phys. B123, 507 (1977); L. Montanet, G. C. Rossi, and G. Veneziano, Phys. Rep. 63, 149 (1980); D. Kharzeev, Phys. Lett. B378, 238 (1996); S. E. Vance, M. Gyulassy, and X. N. Wang, ibid. B443, 45 (1998); V. ToporPop, M. Gyulassy, J. Barrette, C. Gale, X. N. Wang, and N. Xu, Phys. Rev. C 70, 064906 (2004).

[57] B. Andersson, G. Gustafson, and B. Soderberg, Z. Phys. C 20, 317 (1983).

[58] B. Andersson, G. Gustafson, G. Ingelman, and T. Sjostrand, Phys. Rep. 97, 31 (1983).

[59] T. Sjostrand, Comput. Phys. Commun. 82, 74 (1994); program updates and documentation can be found at http://www.thep.lu.se/tf2/staff/torbjorn/Pythia.html.

[60] The Open Standard Codes and Routines (OSCAR) project at http://www-cunuke.phys.columbia.edu/OSCAR/

[61] See EPAPS Document No. (E-PRVCAN-72-781512) for the full source code of the AMPT model in the FORTRAN 77 language and instructions for users. This document can be reached via a direct link in the online article's
HTML reference section or via the EPAPS homepage (http://www.aip.org/pubservs/epaps.html).

[62] D. W. Duke and J. F. Owens, Phys. Rev. D 30, 49 (1984).

[63] S. Y. Li and X. N. Wang, Phys. Lett. B527, 85 (2002).

[64] V. Topor Pop, M. Gyulassy, J. Barrette, C. Gale, X. N. Wang, N. Xu, and K. Filimonov, Phys. Rev. C 68, 054902 (2003).

[65] X. N. Wang and M. Gyulassy, Phys. Rev. Lett. 86, 3496 (2001).

[66] M. Arneodo et al. (European Muon Collaboration), Nucl. Phys. B333, 1 (1990); P. Amaudruz et al. (New Muon Collaboration), Z. Phys. C 51, 387 (1991); M. R. Adams et al. (E665 Collaboration), Phys. Rev. Lett. 68, 3266 (1992).

[67] A. H. Mueller and J. W. Qiu, Nucl. Phys. B268, 427 (1986); J. W. Qiu, ibid. B291, 746 (1987); J. C. Collins and J. Kwiecinski, ibid. B335, 89 (1990); T. Gousset and H. J. Pirner, Phys. Lett. B375, 349 (1996).

[68] M. Gyulassy and X. N. Wang, Nucl. Phys. B420, 583 (1994).

[69] D. Kharzeev and M. Nardi, Phys. Lett. B507, 121 (2001).

[70] J. D. Bjorken, Phys. Rev. D 27, 140 (1983).

[71] M. I. Pavkovic, Phys. Rev. D 13, 2128 (1976).

[72] We have checked numerically that the average $\sqrt{s}$ in AMPT is around $500-600 \mathrm{MeV}$, which is comparable to the default value of $\mu=3 \mathrm{fm}^{-1}$ but not larger. Eq. (8) thus might be oversimplified.

[73] T. S. Biro, P. Levai, and J. Zimanyi, Phys. Lett. B347, 6 (1995); T. S. Biro, hep-ph/0005067; J. Zimanyi, T. S. Biro, T. Csorgo, and P. Levai, Phys. Lett. B472, 243 (2000); J. Zimanyi, P. Levai, T. Csorgo, and T. S. Biro, hep-ph/0103156.

[74] The general Lund flavor-dependent symmetric fragmentation function involves two $a$ parameters which are different for quark and diquark fragmentation/production, while the $b$ parameter is universal. As in the default PYTHIA model, the $a$ parameter for diquarks is larger than that for quarks by 0.5 in the AMPT model. Equation (9) is the simplified expression, where $a$ corresponds to quark production in a $q \bar{q}$ string.

[75] J. S. Schwinger, Phys. Rev. 128, 2425 (1962).

[76] For central $\mathrm{Pb}+\mathrm{Pb}$ collisions at the SPS (see Figs. 16 and 17), we find that with the formation time increased from 0.7 to $1.2 \mathrm{fm} / \mathrm{c}$ in the default AMPT model, the rapidity distributions of negative hadrons and pions are slightly higher (by about $3 \%$ on the average), protons change within about $3 \%$, kaons are lower by about $5 \%$, and antiprotons are lower by about $10 \%$. The decrease in kaons and increase in pions are due to fewer hadronic interactions which produce strangeness, and the decrease in antiprotons is due to a smaller diffusion of baryons into the midrapidity region.

[77] We have checked numerically that the violation of total energy in any event with string melting is between $-0.4 \%$ and $1.7 \%$ for 5000 minimum-bias $\mathrm{Au}+\mathrm{Au}$ events at $\sqrt{s_{N N}}=$ $130 \mathrm{GeV}$. However, the violation in transverse energy could be much larger. For example, take $\delta y \sim 6,\left\langle E_{T}\right\rangle \sim 0.5 \mathrm{GeV}$, and $d N_{\text {total }} / d y \sim 1350$ for central $\mathrm{Au}+\mathrm{Au}$ collisions at $\sqrt{s_{N N}}=$ $200 \mathrm{GeV}$ and attribute all the violation in total energy to the transverse direction for a worst-case estimate, then a $1 \%$ violation (or $400 \mathrm{GeV}$ ) in total energy translates to $67 \mathrm{GeV}$ violation in transverse energy at midrapidity, a violation of up to $10 \%$.

[78] B. A. Li, A. T. Sustich, B. Zhang, and C. M. Ko, Int. J. Mod. Phys. E 10, 267 (2001).

[79] B. A. Li, C. M. Ko, and W. Bauer, Int. J. Mod. Phys. E 7, 147 (1998). 
[80] G. Q. Li, C. M. Ko, and G. E. Brown, Phys. Rev. Lett. 75, 4007 (1995); G. Q. Li, C. M. Ko, and G. E. Brown, Nucl. Phys. A606, 568 (1996); C. M. Ko and G. Q. Li, J. Phys. G 22, 1673 (1996); W. Cassing and E. L. Bratkovskaya, Phys. Rep. 308, 65 (1999); R. Rapp and J. Wambach, Adv. Nucl. Phys. 25, 1 (2000).

[81] G. Song, B. A. Li, and C. M. Ko, Nucl. Phys. A646, 481 (1999).

[82] J. Cugnon, P. Deneye, and J. Vandermeulen, Phys. Rev. C 41, 1701 (1990).

[83] C. M. Ko, Phys. Rev. C 23, 2760 (1981).

[84] G. E. Brown, C. M. Ko, Z. G. Wu, and L. H. Xia, Phys. Rev. C 43, 1881 (1991).

[85] G. J. Wang, R. Bellwied, C. Pruneau, and G. Welke, nuclth/9806006; nucl-th/9807036.

[86] C. M. Ko and R. Yuan, Phys. Lett. B192, 31 (1987).

[87] P. Koch, B. Muller, and J. Rafelski, Phys. Rep. 142, 167 (1986).

[88] C. H. Li and C. M. Ko, Nucl. Phys. A712, 110 (2002).

[89] S. Pal, C. M. Ko, J. M. Alexander, P. Chung, and R. A. Lacey, Phys. Lett. B595, 158 (2004).

[90] W. S. Chung, G. Q. Li, and C. M. Ko, Nucl. Phys. A625, 347 (1997).

[91] C. M. Ko and B. H. Sa, Phys. Lett. B258, 6 (1991).

[92] C. M. Ko and D. Seibert, Phys. Rev. C 49, 2198 (1994).

[93] L. Alvarez-Ruso and V. Koch, Phys. Rev. C 65, 054901 (2002).

[94] H. J. Behrend et al., Phys. Lett. B56, 408 (1975).

[95] H. Joos, Phys. Lett. B24, 103 (1967).

[96] We also found [47] that using $8.3 \mathrm{mb}$ as the elastic cross sections of the $\phi$ meson increases only slightly the slope parameter when compared to results using 0.56 and $2 \mathrm{mb}$, respectively, for the $\phi$ meson elastic cross section with a nucleon and a meson.

[97] W. Liu, C. M. Ko, and L. W. Chen, nucl-th/0505075.

[98] In AMPT calculations on $p p$ and $p \bar{p}$ collisions in Sec. III, the hadron cascade was turned off. We checked that results on the observables shown in Sec. III remain the same after hadronic scatterings were included. Note that all partonic interactions were, however, still included.

[99] G. J. Alner et al. (UA5 Collaboration), Z. Phys. C 33, 1 (1986).

[100] M. Aguilar-Benitez et al., Z. Phys. C 50, 405 (1991).

[101] V. Blobel et al. (Bonn-Hamburg-Munich Collaboration), Nucl. Phys. B69, 454 (1974).

[102] T. Alexopoulos et al., Phys. Rev. Lett. 64, 991 (1990).

[103] T. Alexopoulos et al., Phys. Rev. Lett. 60, 1622 (1988).

[104] F. Abe et al. (CDF Collaboration), Phys. Rev. D 41, 2330 (1990).

[105] S. V. Afanasiev et al. (NA49 Collaboration), Phys. Rev. C 66, 054902 (2002).

[106] T. Alexopoulos et al. (E735 Collaboration), Phys. Rev. D 48, 984 (1993).

[107] B. Alper et al. (British-Scandinavian Collaboration), Nucl. Phys. B87, 19 (1975).

[108] H. Appelshauser et al. (NA49 Collaboration), Phys. Rev. Lett. 82, 2471 (1999).

[109] J. Bachler et al. (NA49 Collaboration), Nucl. Phys. A661, 45 (1999).

[110] B. B. Back et al. (PHOBOS Collaboration), Phys. Rev. Lett. 85, 3100 (2000).

[111] B. B. Back et al. (PHOBOS Collaboration), Phys. Rev. C 65, 031901(R) (2002).
[112] B. B. Back et al. (PHOBOS Collaboration), Phys. Rev. Lett. 87, 102303 (2001); 88, 022302 (2002).

[113] I. G. Bearden et al. (BRAHMS Collaboration), Phys. Rev. Lett. 94, 162301 (2005).

[114] I. G. Bearden et al. (BRAHMS Collaborations), Phys. Lett. B523, 227 (2001).

[115] I. G. Bearden et al. (BRAHMS Collaboration), Phys. Rev. Lett. 88, 202301 (2002).

[116] X. N. Wang and M. Gyulassy, Phys. Rev. Lett. 68, 1480 (1992).

[117] S. Jeon and J. Kapusta, Phys. Rev. C 63, 011901(R) (2000).

[118] X. N. Wang, Nucl. Phys. A698, 296 (2002).

[119] X. N. Wang, Nucl. Phys. A702, 238 (2002).

[120] L. Ahle et al. (E866 Collaboration), Phys. Lett. B476, 1 (2000); B490, 53 (2000).

[121] J. L. Klay et al. (E-0895 Collaboration), Phys. Rev. C 68, 054905 (2003).

[122] L. Ahle et al. (E802 Collaboration), Phys. Rev. C 57, 466 (1998); 58, 3523 (1998); Phys. Rev. Lett. 81, 2650 (1998).

[123] S. S. Adler et al. (PHENIX Collaboration), Phys. Rev. C 69, 034909 (2004).

[124] K. Adcox et al. (PHENIX Collaboration), Phys. Rev. Lett. 89, 092302 (2002); Phys. Rev. C 69, 024904 (2004).

[125] C. Adler et al. (STAR Collaboration), Phys. Lett. B595, 143 (2004).

[126] J. Adams et al. (STAR Collaboration), Phys. Rev. Lett. 92, 112301 (2004).

[127] Data on the $K^{+} / \pi^{+}$ratio around the laboratory energy of $30 \mathrm{~A} \mathrm{GeV}$ show interesting structures [105]; however, a detailed study using the AMPT model has not been done. Because the mean-field potentials at these energies may significantly affect the $K / \pi$ ratio [14], they need to be included in the AMPT model before this issue can be addressed in detail.

[128] I. G. Bearden et al. (BRAHMS Collaboration), Phys. Rev. Lett. 93, 102301 (2004).

[129] K. Adcox et al. (PHENIX Collaboration), Phys. Rev. Lett. 88, 242301 (2002).

[130] G. Q. Li and C. M. Ko, Nucl. Phys. A582, 731 (1995).

[131] W. S. Chung, C. M. Ko, and G. Q. Li, Nucl. Phys. A641, 357 (1998).

[132] M. Asakawa and C. M. Ko, Nucl. Phys. A572, 732 (1994).

[133] C. M. Ko, V. Koch, and G. Q. Li, Annu. Rev. Nucl. Part. Sci. 47, 505 (1997).

[134] S. V. Afanasev et al. (NA49 Collaboration), Phys. Lett. B491, 59 (2000).

[135] M. C. Abreu et al. (NA50 Collaboration), J. Phys. G 27, 405 (2001); and references therein.

[136] B. Muller and J. Rafelski, Phys. Lett. B101, 111 (1981).

[137] Z. W. Lin and C. M. Ko, Phys. Rev. Lett. 89, 202302 (2002).

[138] S. A. Voloshin, Nucl. Phys. A715, 379 (2003).

[139] D. Molnar and S. A. Voloshin, Phys. Rev. Lett. 91, 092301 (2003).

[140] R. J. Fries, B. Muller, C. Nonaka, and S. A. Bass, Phys. Rev. Lett. 90, 202303 (2003).

[141] V. Greco, C. M. Ko, and P. Levai, Phys. Rev. Lett. 90, 202302 (2003).

[142] Z. W. Lin and D. Molnar, Phys. Rev. C 68, 044901 (2003).

[143] V. Greco, C. M. Ko, and R. Rapp, Phys. Lett. B595, 202 (2004).

[144] V. Greco, C. M. Ko, and P. Levai, Phys. Rev. C 68, 034904 (2003). 
[145] R. J. Fries, B. Muller, C. Nonaka, and S. A. Bass, Phys. Rev. C 68, 044902 (2003).

[146] T. Matsui and H. Satz, Phys. Lett. B178, 416 (1986).

[147] O. Kaczmarek, F. Karsch, E. Laermann, and M. Lutgemeier, Phys. Rev. D 62, 034021 (2000).

[148] F. Karsch, Nucl. Phys. A698, 199 (2002).

[149] V. Bornyakov et al., Nucl. Phys. Proc. Suppl. 119, 703 (2003).

[150] D. Kharzeev, C. Lourenco, M. Nardi, and H. Satz, Z. Phys. C 74, 307 (1997).

[151] U. W. Heinz and M. Jacob, nucl-th/0002042.

[152] D. M. Alde et al., Phys. Rev. Lett. 66, 133 (1991).

[153] M. J. Leitch et al. (FNAL E866/NuSea Collaboration), Phys. Rev. Lett. 84, 3256 (2000).

[154] M. C. Abreu et al. (NA50 Collaboration), Phys. Lett. B410, 337 (1997).

[155] E. V. Shuryak, Phys. Lett. B78, 150 (1978).

[156] X. M. Xu, D. Kharzeev, H. Satz, and X. N. Wang, Phys. Rev. C 53, 3051 (1996).

[157] S. Gavin and R. Vogt, Phys. Rev. Lett. 78, 1006 (1997).

[158] W. Cassing and C. M. Ko, Phys. Lett. B396, 39 (1997).

[159] J. Geiss, C. Greiner, E. L. Bratkovskaya, W. Cassing, and U. Mosel, Phys. Lett. B447, 31 (1999).

[160] D. E. Kahana and S. H. Kahana, Phys. Rev. C 59, 1651 (1999).

[161] C. Spieles, R. Vogt, L. Gerland, S. A. Bass, M. Bleicher, H. Stocker, and W. Greiner, Phys. Rev. C 60, 054901 (1999).

[162] B. H. Sa, A. Tai, H. Wang, and F. H. Liu, Phys. Rev. C 59, 2728 (1999).

[163] C. Spieles et al., Phys. Lett. B458, 137 (1999).

[164] A. Capella, E. G. Ferreiro, and A. B. Kaidalov, Phys. Rev. Lett. 85, 2080 (2000).

[165] K. L. Haglin, Phys. Rev. C 61, 031902(R) (2000).

[166] Z. W. Lin and C. M. Ko, Phys. Rev. C 62, 034903 (2000).

[167] W. Liu, C. M. Ko, and Z. W. Lin, Phys. Rev. C 65, 015203 (2002).

[168] A. Sibirtsev, K. Tsushima, and A. W. Thomas, Phys. Rev. C 63, 044906 (2001).

[169] F. S. Navarra, M. Nielsen, R. S. Marques de Carvalho, and G. Krein, Phys. Lett. B529, 87 (2002).

[170] C. Y. Wong, E. S. Swanson, and T. Barnes, Phys. Rev. C 62, 045201 (2000).

[171] C. Y. Wong, T. Barnes, E. S. Swanson, and H. W. Crater, nucl-th/0112023.

[172] R. L. Thews, M. Schroedter, and J. Rafelski, Phys. Rev. C 63, 054905 (2001).

[173] P. Braun-Munzinger and J. Stachel, Phys. Lett. B490, 196 (2000).

[174] P. Braun-Munzinger and J. Stachel, Nucl. Phys. A690, 119 (2001).

[175] L. Grandchamp and R. Rapp, Phys. Lett. B523, 60 (2001).

[176] The current versions of the AMPT model (v1.11 and v2.11) do not include the option of treating $J / \psi$ dynamics.

[177] S. Batsouli, S. Kelly, M. Gyulassy, and J. L. Nagle, Phys. Lett. B557, 26 (2003).

[178] J. Barrette et al. (E877 Collaboration), Phys. Rev. Lett. 73, 2532 (1994).

[179] H. Appelshauser et al. (NA49 Collaboration), Phys. Rev. Lett. 80, 4136 (1998).

[180] J. Y. Ollitrault, Phys. Rev. D 46, 229 (1992).

[181] H. Sorge, Phys. Rev. Lett. 78, 2309 (1997); 82, 2048 (1999).

[182] P. Danielewicz, R. A. Lacey, P. B. Gossiaux, C. Pinkenburg,
P. Chung, J. M. Alexander, and R. L. McGrath, Phys. Rev. Lett. 81, 2438 (1998).

[183] Y. M. Zheng, C. M. Ko, B. A. Li, and B. Zhang, Phys. Rev. Lett. 83, 2534 (1999).

[184] K. H. Ackermann et al. (STAR Collaboration), Phys. Rev. Lett. 86, 402 (2001).

[185] R. A. Lacey (PHENIX Collaboration), Nucl. Phys. A698, 559 (2002).

[186] I. C. Park et al. (PHOBOS Collaboration), Nucl. Phys. A698, 564 (2002).

[187] R. J. Snellings (STAR Collaboration), Nucl. Phys. A698, 193 (2002).

[188] B. B. Back et al. (PHOBOS Collaboration), Phys. Rev. Lett. 89, 222301 (2002).

[189] C. Adler et al. (STAR Collaboration), Phys. Rev. C 66, 034904 (2002).

[190] K. Adcox (PHENIX Collaboration), Phys. Rev. Lett. 89, 212301 (2002).

[191] C. Roland et al. (PHOBOS Collaboration), Nucl. Phys. A698, 54 (2002).

[192] C. Adler et al. (STAR Collaboration), Phys. Rev. Lett. 87, 182301 (2001).

[193] H. Heiselberg and R. Mattiello, Phys. Rev. C 60, 044902 (1999).

[194] M. Gyulassy, I. Vitev, and X. N. Wang, Phys. Rev. Lett. 86, 2537 (2001).

[195] X. N. Wang, Phys. Rev. C 63, 054902 (2001).

[196] H. Heiselberg and A. M. Levy, Phys. Rev. C 59, 2716 (1999).

[197] P. Huovinen, P. F. Kolb, and U. W. Heinz, Nucl. Phys. A698, 475 (2002).

[198] T. J. Humanic, Nucl. Phys. A715, 641 (2003).

[199] E. E. Zabrodin, C. Fuchs, L. V. Bravina, and A. Faessler, Phys. Lett. B508, 184 (2001).

[200] B. Zhang, M. Gyulassy, and C. M. Ko, Phys. Lett. B455, 45 (1999).

[201] D. Molnar and M. Gyulassy, Nucl. Phys. A698, 379 (2002); A697, 495 (2002); A703, 893(E) (2002).

[202] D. Teaney, J. Lauret, and E. V. Shuryak, Phys. Rev. Lett. 86, 4783 (2001); Nucl. Phys. A698, 479 (2002).

[203] C. Adler et al. (STAR Collaboration), Phys. Rev. Lett. 89, 132301 (2002).

[204] C. Adler et al. (STAR Collaboration), Phys. Rev. Lett. 90, 032301 (2003).

[205] B. B. Back et al. (PHOBOS Collaboration), nucl-ex/0407012.

[206] P. F. Kolb, L. W. Chen, V. Greco, and C. M. Ko, Phys. Rev. C 69, 051901(R) (2004).

[207] P. Sorensen (STAR Collaboration), J. Phys. G 30,S217(2004).

[208] S. S. Adler et al. (PHENIX Collaboration), Phys. Rev. Lett. 91, 182301 (2003).

[209] V. Greco and C. M. Ko, Phys. Rev. C 70, 024901 (2004).

[210] X. Dong, S. Esumi, P. Sorensen, N. Xu, and Z. Xu, Phys. Lett. B597, 328 (2004).

[211] L. W. Chen, V. Greco, C. M. Ko, and P. F. Kolb, Phys. Lett. B605, 95 (2005). Note that a cutoff time $t_{\text {cut }}$ of $500 \mathrm{fm} / c$ is used for hadron cascade in this study of the pseudorapidity dependence of $v_{2}$.

[212] L. W. Chen, C. M. Ko, and Z. W. Lin, Phys. Rev. C 69, 031901(R) (2004).

[213] J. Adams et al. (STAR Collaboration), Phys. Rev. Lett. 92, 062301 (2004).

[214] R. Hanbury Brown and R. Q. Twiss, Nature 178, 1046 (1956). 
[215] S. Pratt, Phys. Rev. Lett. 53, 1219 (1984).

[216] G. Bertsch, M. Gong, and M. Tohyama, Phys. Rev. C 37, 1896 (1988).

[217] S. Pratt, T. Csörgö, and J. Zimanyi, Phys. Rev. C 42, 2646 (1990).

[218] D. H. Rischke and M. Gyulassy, Nucl. Phys. A608, 479 (1996).

[219] S. Soff, S. A. Bass, and A. Dumitru, Phys. Rev. Lett. 86, 3981 (2001).

[220] S. Soff, S. A. Bass, D. H. Hardtke, and S. Y. Panitkin, Phys. Rev. Lett. 88, 072301 (2002).

[221] C. Adler et al. (STAR Collaboration), Phys. Rev. Lett. 87, 082301 (2001).

[222] S. C. Johnson (PHENIX Collaboration), Nucl. Phys. A698, 603 (2002)

[223] K. Adcox et al. (PHENIX Collaboration), Phys. Rev. Lett. 88, 192302 (2002).

[224] U. W. Heinz and P. F. Kolb, in Proceedings of the 18th Winter Workshop on Nuclear Dynamics, edited by R. Bellwied, J. Harris, and W. Bauer (EP Systema, Debrecen, Hungary, 2002), p. 205.

[225] U. A. Wiedemann and U. W. Heinz, Phys. Rep. 319, 145 (1999).

[226] J. P. Sullivan, M. Berenguer, B. V. Jacak, S. Pratt, M. Sarabura, J. Simon-Gillo, H. Sorge, and H. van Hecke, Phys. Rev. Lett. 70, 3000 (1993).

[227] D. Hardtke and S. A. Voloshin, Phys. Rev. C 61, 024905 (2000).

[228] S. Pratt et al., Nucl. Phys. A566, 103c (1994).

[229] J. Adams et al. (STAR Collaboration), Phys. Rev. Lett. 93, 012301 (2004).

[230] B. B. Back et al. (PHOBOS Collaboration), nucl-ex/0409001, Phys. Rev. C (to be published).
[231] D. A. Brown and P. Danielewicz, Phys. Lett. B398, 252 (1997); Phys. Rev. C 57, 2474 (1998); 64, 014902 (2001).

[232] S. Y. Panitkin and D. A. Brown, Phys. Rev. C 61, 021901(R) (1999).

[233] Z. W. Lin and U. W. Heinz, in preparation.

[234] Y. Schutz, J. Phys. G 30, S903 (2004).

[235] N. Armesto, C. A. Salgado, and U. A. Wiedemann, Phys. Rev. Lett. 94, 022002 (2005).

[236] D. Kharzeev, E. Levin, and M. Nardi, Nucl. Phys. A747, 609 (2005).

[237] A. D. Martin, W. J. Stirling, and R. G. Roberts, Phys. Rev. D 50, 6734 (1994).

[238] S. Kretzer, H. L. Lai, F. I. Olness, and W. K. Tung, Phys. Rev. D 69, 114005 (2004).

[239] M. Gluck, E. Reya, and A. Vogt, Z. Phys. C 67, 433 (1995).

[240] Z. W. Lin and C. M. Ko, Nucl. Phys. A671, 567 (2000).

[241] C. Greiner and Z. Xu, hep-ph/0406027.

[242] D. Molnar's contribution in S. A. Bass et al., Nucl. Phys. A661 205 (1999).

[243] Y. Pang, RHIC 96 Summer Study, CU-TP-815 preprint (unpublished); Generic cascade program (GCP) (documentation available at the OSCAR Website [60].)

[244] B. Zhang, M. Gyulassy, and Y. Pang, Phys. Rev. C 58, 1175 (1998).

[245] S. Cheng, S. Pratt, P. Csizmadia, Y. Nara, D. Molnár, M. Gyulassy, S. E. Vance, and B. Zhang, Phys. Rev. C 65, 024901 (2002).

[246] B. B. Back et al. (PHOBOS Collaboration), Phys. Rev. Lett. 93, 082301 (2004).

[247] J. Adams et al. (STAR Collaboration), Phys. Rev. C 70, 064907 (2004).

[248] F. Simon (STAR Collaboration), J. Phys. G 31, S1065 (2005). 